\title{
Modelos multivariados binários com funções de ligação assimétricas
}

\author{
Rafael Bráz Azevedo Farias
}

TESE APRESENTADA

$\mathrm{AO}$

Instituto DE MATEMÁticA E EstatísticA

DA

UNIVERSIDADE DE SÃO PAULO

PARA

OBTENÇÃO DO TÍTULO

$\mathrm{DE}$

DOUTOR EM CIÊNCIAS

Programa: Estatística

Orientador: Prof. Dra. Márcia D`Elia Branco

Durante o desenvolvimento deste tese o autor recebeu auxílio financeiro do CNPq e da FAPESP

São Paulo, Maio de 2012 


\title{
Modelos multivariados binários com funções de ligação assimétricas
}

\author{
Esta tese contém as correções e alterações \\ sugeridas pela Comissão Julgadora durante a defesa \\ realizada por Rafael Bráz Azevedo Farias em 25/05/2012. \\ O original encontra-se disponível no Instituto de \\ Matemática e Estatística da Universidade de São Paulo.
}

Comissão Julgadora:

- Profa. Dra. Márcia D‘Elia Branco (orientadora) - IME-USP

- Prof. Dra. Rosângela Helena Loschi - UFMG

- Prof. Dr. Reinaldo Boris Arellano Valle - PUC-Chile

- Prof. Dr. Jorge Luis Bazán Guzmán - PUC-Perú

- Prof. Dr. Heleno Bolfarine - IME-USP 


\section{Agradecimentos}

Aos meus pais, Afonso e Fátima, pelo amor, carinho, e por me apoiarem em minhas difíceis decisões. Muito obrigado meus queridos!

À minha irmã Daniele pelo apoio, brincadeiras de infância e conselhos na vida adulta. À minha priminha Cinthia (agora não tão pequena) por todo carinho.

À Gisele pelo apoio, carinho e por me compreender nas inúmeras vezes que a tese não me permitia sair nos finais de semana.

À minha orientadora Márcia D'Elia Branco, pela confiança, conselhos, ajuda e pela ótima orientação que recebi ao longo do desenvolvimento deste trabalho. Muito obrigado!

Ao professor Dipak Dey por sua orientação e acolhida durante meu estágio no exterior. Aos funcionários e colegas do departamento de Estatística de UCONN e do International Center de UCONN pela ajuda e hospitalidade.

Aos professores do Departamento de Estatística do IME-USP pelos valiosos ensinamentos recebidos, principalmente à Mônica Sandoval, Júlia Pavan, Chang Chiann, Anatoli Iambartsev, Gilberto Alvarenga, Márcia Branco, Silvia Ferrari, Antonio Carlos, Adilson Simonis, Viviane Giampaoli, Sergio Wechsler, Carlinhos Pereira e Júlio Singer pelos cursos ministrados. Aos funcionários da USP pela prontidão em diversos momentos e esclarecimentos prestados.

Aos professores do Departamento de Estatística e Matemática Aplicada da UFC por me fornecerem uma base sólida na minha caminhada, especialmente aos professores João Maurício e João Welliandre.

Aos meus amigos e colegas da Àrea de Modelagem do Departamento de Controle de Riscos do Banco Bradesco, principalmente aos colegas Luciano Valiensi e Danilo Damasceno pelas várias tardes que permitiram que eu me ausentasse para terminar esta tese.

Aos amigos Fabio Demarqui, Marcos Prates, Jinhyouk Jung, Victor Hugo Lachos e família por tornarem minha estada nos Estados Unidos bem mais agradável.

Aos amigos de graduação Ênio Lopes, Chagas Almeida, Michel Helcias, Fabienne Rodrigues e Eveliny Barroso, pelas conversas e pelos inúmeros momentos agradáveis. Aos amigos Juvêncio 
Nobre, Jacqueline Batista, Caio Azevedo, Iesus Diniz e Márcio Medeiros, pela acolhida durante o primeiro ano de mestrado, e pelos momentos de descontração e aprendizado.

Aos colegas do IME-USP pelo companheirismo, pela ajuda nas horas difíceis e pelos momentos de descontração, dentre os quais destaco: Germán Moreno, Artur Lemonte, Michel Helcias, Tiago Magalhães, Nubia Esteban, Luz Marina, Betsabé Grimalda, Amanda Gomes, Jalmar Farvan, Ivan Enriquez, Francisco Marcelo, Frederico Poleto, Eliardo e Gustavo.

Aos meus tios Cleide, Milton, Zuleide e Chico Mendes, pelo carinho, compreensão e amizade durante minha infância e adolescência.

A todos os amigos e colegas de Fortaleza, São Paulo e Connecticut-EUA que de alguma forma contribuíram para esse momento.

Aos membros da banca examinadora pelas sugestões e comentários valiosos para o aprimoramento do texto.

Ao CNPq pelo apoio financeiro nos três primeiros meses do doutorado e à FAPESP pelo suporte financeiro concedido por um período de quase quatro anos, possibilitanto minha ida para os Estados Unidos da América. 


\section{Resumo}

\section{Modelos multivariados binários com funções de ligação assimétricas}

Conjuntos de dados com respostas multivariadas aparecem frequentemente em pesquisas em que os dados são provenientes de questionários. As pesquisas de opinião são exemplos comuns de estudos em que as respostas são multivariadas. Um tipo pesquisa de opinião que ganha grande destaque no Brasil em anos de eleições, é a pesquisa de inteção de votos. Entretanto, apesar da maior visibilidade dos estudos de prognósticos eleitorais, a pesquisa de opinião pública é uma ferramenta amplamente utilizada para detectar tendências e posições dos diferentes segmentos sociais em diversos temas, sejam eles, políticos, sociais e governamentais. Nós introduzimos nesta tese uma classe de modelos de regressão multivariados com funções de ligação assimétricas para o ajuste de conjuntos de dados com respostas multivariadas binárias. As funções de ligação consideradas são bastante flexíveis e robustas, contemplando funções de ligação simétricas como casos particulares. Devido a complexidade do modelo, nós discutimos a sua identificabilidade. A abordagem Bayesiana foi considerada e alguns algoritmos de Monte Carlo via Cadeia de Markov (MCMC) foram desenvolvidos. Estudos de simulação foram desenvolvidos com dois objetivos; i) verificar a qualidade dos algoritmos desenvolvidos e ii) verificar a importância da escolha da função de ligação. No final da tese uma aplicação em um conjunto de dados real é considerada com o objetivo de ilustrar as metodologias e técnicas apresentadas.

Palavras-chave: modelos binários, regressão multivariada, distribuições elípticas assimétricas. 


\section{Abstract}

\section{Multivariate binary models with skewed link functions}

Data sets with multivariate responses often appear in surveys where the data came from questionnaires. Opinion poll, sometimes simply referred to as a poll, are common examples of studies in which the responses are multivariate. One type poll that gain great prominence in Brazil in election years, is the survey of vote intent. However, despite the higher visibility of prognostic studies of election, opnion polls is a tool widely used to detect trends and positions of different social segments on various topics, be they political, social or governmental. We introduce in this work a class of multivariate regression models with asymmetric link functions to fit data sets with multivariate binary responses. The link functions here considered are quite flexible and robust, contemplating symmetrical link functions as special cases. Due to the complexity of the model, we discuss its identifiability. The Bayesian approach was considered and some Monte Carlo Markov Chain (MCMC) algorithms have been developed. Simulation studies have been developed with two objectives: i) verify the quality of the algorithms developed and ii) to verify the importance of choosing the link function. At the end of this work an application in a real data set is considered in order to illustrate the methodologies and techniques presented.

Keywords: binary models, multivariate regression models, skew-elliptical distributions. 


\section{Sumário}

$\begin{array}{ll}\text { Lista de Abreviaturas } & \text { ix }\end{array}$

Lista de Figuras $\quad$ xi

Lista de Tabelas $\quad$ xiii

1 Introdução $\quad 1$

1.1 Organização do Trabalho . . . . . . . . . . . . . . . . . . . . 4

2 Modelos Multivariados Binários $\quad 7$

2.1 Modelo multivariado com função de ligação elíptica . . . . . . . . . . . . . . . . . 10

2.2 Modelo multivariado com função de ligação elíptica assimétrica . . . . . . . . . . . 14

2.2 .1 Exemplos de distribuições assimétricas . . . . . . . . . . . . . . . . . 17

3 Inferência em Modelos Assimétricos $\quad 21$

3.1 Identificabilidade do modelo . . . . . . . . . . . . . . . . . . . . 22

3.1.1 Casos particulares de falta de identificabilidade . . . . . . . . . . . 25

3.2 Algoritmos MCMC . . . . . . . . . . . . . . . . . . 26

3.3 Distribuição condicional completa dos coeficientes da regressão . . . . . . . . . . . 29

3.4 Distribuição condicional completa da matriz de dependência . . . . . . . . . . . . . . . . . . . . . . 39

3.4.1 Expansão paramétrica para dados aumentados . . . . . . . . . . . . 30

3.5 Distribuição condicional completa da variável de mistura . . . . . . . . . . . . . . 33

4 Estudos de Simulação $\quad 37$

4.1 Estudo de simulação I . . . . . . . . . . . . . . . . . . . . . . . . . . . 39

4.2 Estudo de simulação II . . . . . . . . . . . . . . . . . . . . . . . . . 45

5 Aplicação $\quad 49$

5.1 Comparação de modelos . . . . . . . . . . . . . . . . . . . . . . 52

5.2 Diagnóstico de influência . . . . . . . . . . . . . . . . . . 55

6 Comentários Finais $\quad 65$

$\begin{array}{ll}\text { A Seleção de Modelos } & 67\end{array}$

A.1 Medidas de comparação de modelos . . . . . . . . . . . . . . . . . . . . . . . . . . . . . 67

A.2 Análise de observações influentes . . . . . . . . . . . . . . . . . . 70 
viii SUMÁRIO

Referências Bibliográficas 


\title{
Lista de Abreviaturas
}

\author{
MCMC Monte Carlo via Cadeias de Markov (Monte Carlo Markov Chain) \\ MEN Mistura de Escala de Normais \\ MENA Mistura de Escala de Normais Assimétricas \\ MLG Modelos Lineares Generalizados \\ MMP Modelo Multivariado Probito \\ MMT Modelo Multivariado t-Student \\ MML Modelo Multivariado Logístico \\ MMC Modelo Multivariado Cauchy \\ MMED Modelo Multivariado Exponêncial Duplo \\ MMEP Modelo Multivariado Exponêncial Potência \\ MMSL Modelo Multivariado Slash \\ MMS Modelo Multivariado Stable \\ MMPA Modelo Multivariado Probito Assimétrico \\ MMTA Modelo Multivariado t-Student Assimétrico \\ MMLA Modelo Multivariado Logístico Assimétrico \\ MMCA Modelo Multivariado Cauchy Assimétrico \\ MMEDA Modelo Multivariado Exponêncial Duplo Assimétrico \\ MMEPA Modelo Multivariado Exponêncial Potência Assimétrico \\ MMSLA Modelo Multivariado Slash Assimétrico \\ MMSA Modelo Multivariado Stable Assimétrico \\ PX-DA Expansão Paramétrica para Dados Aumentados
}




\section{Lista de Figuras}

1.1 Modelos probito e cloglog ajustados à proporção de besouros mortos. . . . . . . . . . 4

4.1 Boxplots das distribuições a posteriori de $\beta_{11} \operatorname{com} \delta$ e $n$ variando. . . . . . . . . . . 42

4.2 Boxplots das distribuições a posteriori de $\beta_{12} \operatorname{com} \delta$ e $n$ variando. . . . . . . . . . . 43

4.3 Boxplots das distribuições a posteriori de $\beta_{21} \operatorname{com} \delta$ e $n$ variando. . . . . . . . . . 43

4.4 Boxplots das distribuições a posteriori de $\beta_{22} \operatorname{com} \delta$ e $n$ variando. . . . . . . . . . . 44

4.5 Boxplots das distribuições a posteriori de $\sigma_{12} \operatorname{com} \delta$ e $n$ variando. . . . . . . . . 44

5.1 Cadeias de Markov das distribuições a posteriori dos parâmetros de interesse. . . . . 51

5.2 Estimativas pontuais das correlações (a) e gráficos boxplot das distribuições a posteriori das correlações $(\mathrm{b}) \ldots \ldots \ldots \ldots \ldots$. . . . . . . . . . . . . 52

5.3 Estimativas pontuais das correlações (a) e gráficos boxplot das distribuições a posteriori das correlações (b) considerando o modelo probito assimétrico. . . . . . . . . 58

5.4 O inverso do CPO aplicado ao conjunto de dados sobre o comportamento de votos na cidade de Troy para os modelos $\mathcal{M}_{1}, \mathcal{M}_{2}, \mathcal{M}_{3}, \mathcal{M}_{4}, \mathcal{M}_{5}$ e $\mathcal{M}_{6}$. . . . . . . . . 59

5.5 O inverso do CPO aplicado ao conjunto de dados sobre o comportamento de votos na cidade de Troy para os modelos $\mathcal{M}_{7}, \mathcal{M}_{8}, \mathcal{M}_{9}, \mathcal{M}_{10}, \mathcal{M}_{21}$ e $\mathcal{M}_{40} \ldots$. . . . . . 60

5.6 A divergência K-L aplicada ao conjunto de dados sobre o comportamento de votos na cidade de Troy para os modelos $\mathcal{M}_{1}, \mathcal{M}_{2}, \mathcal{M}_{3}, \mathcal{M}_{4}, \mathcal{M}_{5}$ e $\mathcal{M}_{6} . \ldots \ldots$. . . . 61

5.7 A divergência K-L aplicada ao conjunto de dados sobre o comportamento de votos na cidade de Troy para os modelos $\mathcal{M}_{7}, \mathcal{M}_{8}, \mathcal{M}_{9}, \mathcal{M}_{10}, \mathcal{M}_{21}$ e $\mathcal{M}_{40} \ldots$. . . . . . 62

5.8 A calibração da divergência K-L aplicada ao ao conjunto de dados sobre o comportamento de votos na cidade de Troy para os modelos $\mathcal{M}_{1}$ e $\mathcal{M}_{11} \ldots \ldots \ldots$. . . . . 63 


\section{Lista de Tabelas}

2.1 Função geradora de densidade de algumas distribuições elípticas. . . . . . . . . . . . . 11

2.2 Algumas funções de mistura e suas respectivas variáveis misturadoras . . . . . . . . . 20

4.1 Valores médios dos parâmetros estimados para cada configuração de parâmetro de forma e tamanho de amostra. . . . . . . . . . . . . . . . . . . 41

4.2 Viéses relativos dos parâmetros estimados para cada configuração de parâmetro de forma e tamanho de amostra. . . . . . . . . . . . . . . . . . 41

4.3 Erros quadráticos médios relativos $\left(\mathrm{EQM}_{R}\right)$ dos parâmetros estimados para cada configuração de parâmetro de forma e tamanho de amostra. . . . . . . . . . . . . . . 42

4.4 Valores médios dos parâmetros estimados para cada configuração de parâmetro de forma e tamanho de amostra, considerado $\boldsymbol{\delta}=(0,0)$ no processo de estimação. . . . . 46

4.5 Erro quadrático médio, AIC e BIC para o modelo multivariado probito simétrico. . . 46

4.6 Erro quadrático médio, AIC e BIC para cada configuração de parâmetro de forma e tamanho de amostra para os estudos de simulação I e II . . . . . . . . . . . . . . 47

5.1 Estatísticas a posteriori para o modelo bivariado probito ajustado ao conjunto de dados sobre o comportamento de votos na cidade de Troy. . . . . . . . . . . . . 50

5.2 Configurações do vetores $\boldsymbol{\delta}_{k}=\left(\delta_{k 1}, \delta_{k 2}\right)^{\prime}$ para cada um modelos concorrentes $\mathcal{M}_{k}$,

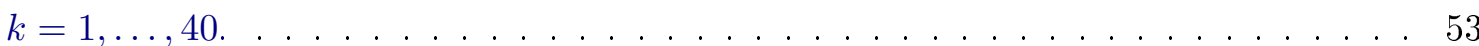

5.3 Critérios de seleção AIC, BIC, $\bar{D}, p_{D}$, DIC, LPML e PsBF para os 40 modelos concorrentes aplicados no conjunto de dados sobre o comportamento de votos na cidade de Troy. . . . . . . . . . . . . . . . . . . . . . . 55

5.4 Valores das medidas de influência das observações influentes para o modelo $\mathcal{M}_{1}$ comparada com as valores obtidos pelo modelo $\mathcal{M}_{10}$ ( $c$ indica que a observação é influente segundo a CPO e $k$ indica que a observação é influente de acordo com a divergência K-L) . . . . . . . . . . . . . . . . . . . 57

5.5 Observações influentes para o modelo $\mathcal{M}_{1}$ e suas respectivas probabilidades ajustadas para os modelos $\mathcal{M}_{1}$ e $\mathcal{M}_{10}$ ( $c$ indica que a observação é influente segundo a $\mathrm{CPO}^{-1}$ e $k$ indica que a observação é influente de acordo com a divergência K-L). . . . . . . 57

5.6 Estatísticas a posteriori para o modelo bivariado probito assimétrico com vetor de parâmetros de forma $\boldsymbol{\delta}=(0,0 ; 0,2)^{\prime}$ ajustado ao conjunto de dados sobre o comportamento de votos na cidade de Troy. . . . . . . . . . . . . . . . 58 


\section{Capítulo 1}

\section{Introdução}

Conjuntos de dados com respostas multivariadas aparecem frequentemente em pesquisas em que os dados são provenientes de questionários. As pesquisas de opinião são exemplos comuns de estudos em que as respostas são multivariadas. Um tipo pesquisa de opinião que ganha grande destaque no Brasil em anos de eleições, é a pesquisa de intenção de votos. Entretanto, apesar da maior visibilidade dos estudos de prognósticos eleitorais, a pesquisa de opinião pública é uma ferramenta amplamente utilizada para detectar tendências e posições dos diferentes segmentos sociais em diversos temas, sejam eles, políticos, sociais e governamentais. Greene (1997) apresenta um conjunto de dados referente a uma pesquisa sobre o comportamento dos votos de residentes da cidade de Troy, no estado de Michigan, Estados Unidos da América. Neste estudo, 95 moradores foram questionados a responder duas perguntas: $1^{a}$ ) se tem ou não, pelo menos um filho na escola pública e; $2^{a}$ ) se vota a favor ou contra a criação de um fundo orçamentário para a escola. O objetivo desta pesquisa foi estudar o comportamento entre as duas variáveis resposta binárias e algumas variáveis explicativas (preditoras). As variáveis explicativas consideradas foram a renda familiar anual, o valor pago por ano em impostos sobre imóveis por cada família e o número de anos que a família reside na cidade de Troy. É importante notar que, uma vez que o mesmo indivíduo responde todos os itens do questionário, é provável que suas respostas sejam correlacionadas. Isto é, o fato de um indivíduo ter filhos na escola deve ser correlacionado com sua disposição a votar a favor ou contra a criação de um fundo orçamentário para a escola. De antemão, pode-se esperar que pessoas com filhos na escola sejam mais propensas a votar na criação de um fundo orçamentário para a escola do que pessoas que não tem filhos na escola.

Os Modelos Lineares Generalizados (MLG) são ferramentas amplamente utilizadas quando existe o interesse em analisar a relação entre as variáveis respostas e as variáveis explicativas (covariáveis), as quais podem ser contínuas ou discretas (nominais ou ordinais). O modelo de regressão logística é o MLG utilizado com maior frequência no ajuste de conjunto de dados com respostas binárias. Um dos motivos desta popularidade é a fácil interpretação de seus parâmetros regressores através de razões de chances. Outros modelos, também amplamente utilizados, são os modelos probito e complemento log-log. No entanto, em muitas aplicações, por exemplo, no estudo sobre o comportamento dos votos de residentes da cidade de Troy apresentado anteriormente, independência entre as variáveis respostas não é uma suposição razoável. Esta falta de independência entre as variáveis resposta é facilmente notado em estudos longitudinais, em que as múltiplas medidas são coletadas do mesmo indivíduo. Uma técnica bastante geral para análise de dados correlacionados é 
a abordagem através das equações de estimação generalizada (EEG) introduzida por Liang e Zeger (1986) e Zeger e Liang (1986). Uma boa revisão de várias estratégias de modelagem utilizando regressão linear generalizada com a abordagem por equações de estimação generalizada para analisar dados binários correlacionados com covariáveis associadas a cada resposta é apresentada em Prentice (1988). A principal desvantagem da abordagem usando equações de estimação generalizadas é que ela é baseada em teoria assintótica, e portanto, um tamanho grande de amostra é necessário.

No contexto Bayesiano, diferentes abordagens para modelos com respostas binárias são apresentadas em Zellner e Rossi (1984) e Albert e Chib (1993). Albert e Chib (1993) propõem o uso de variáveis latentes e do algoritmo de Gibbs (Gelfand e Smith, 1990) na estimação dos parâmetros de modelos com reposta binária, o que torna o modelo computacionalmente mais atrativo. Esta proposta permite definir resíduos latentes contínuos, o que não ocorre na abordagem de estimação via máxima verossimilhança. Farias e Branco (2012) sugerem o uso de diversos tipos de resíduos latentes bayesianos para os modelos probito, logístico e probito-assimétrico. Com o objetivo de obter algoritmos mais eficientes, Cowles (1996) e Nandram e Chen (1996) propõem algoritmos alternativos para acelerar a convergência do algoritmo de Gibbs proposto Albert e Chib (1993). Recentemente, Holmes e Held (2006) propõem alternativas mais eficientes de que o algoritmo de Gibbs convencional no contexto de regressão binária com funções de ligação probito e logito. Os autores utilizam o amostrador de Gibbs em blocos (Liu, 1994) para tornar o algoritmo mais eficiente, reduzindo a autocorrelação serial da amostra simulada. A construção destes novos algoritmos depende da obtenção de formas conhecidas para as distribuições marginais de alguns parâmetros ao invés das distribuições condicionais completas. Farias e Branco (2011) propõem um algoritmo similar para trabalhar com o modelo probito-assimétrico proposto por Chen, Dey e Shao (1999), e mostram que o algoritmo proposto é mais eficiente que o convencional (sem blocos). No entanto, Farias e Branco (2011) não incluíram correlação entre as variáveis respostas.

Chib e Greenberg (1998) propuseram um modelo multivariado probito (MMP) para analisar dados com respostas binárias correlacionadas, por exemplo, dados provenientes de estudos longitudinais. Os autores discutem a implementação deste modelo via inferência bayesiana e o método da máxima verossimilhança. Chen e Dey (1998) estendem o modelo MMP através do uso de uma rica classe de distribuições obtidas através de mistura no parâmetro de escala da distribuição normal (Andrews e Mallows, 1974), a qual contempla os modelos multivariados probito (MMP), $t$-Student (MMT) e logístico (MML). Chen e Dey (2003) discutem o problema de seleção de variáveis nesta abordagem. Outras abordagens alternativas para análise de dados multivariados com resposta binária e função de ligação logística são apresentadas por Carey et al. (1993), le Cessie e van Houwelingen (1994) e Glonek e McCullagh (1974). No entanto, apenas modelos simétricos foram considerados em todas estas abordagens. Ou seja, modelos em que a relação (função de ligação) entre as probabilidades de interesse e as variáveis explicativas é simétrica. Estas funções de ligação simétricas comumente utilizadas, tais como o probito e logito, nem sempre fornecem o melhor ajuste.

O modelo complemento loglog é uma alternativa assimétrica quando a assimetria da curva de resposta é positiva. No caso em que a assimetria da curva de resposta é negativa, torna-se 
necessário fazer uma transformação na variável resposta para o uso do modelo complemento loglog. Contudo, a função de ligação complemento loglog tem uma assimetria fixa, o que prejudica o ajuste quando a assimetria da curva de resposta é muito elevada. Neste contexto, Czado e Santner (1992), Chen (2004), Bázan, Branco e Bolfarine (2005) e Wang e Dey (2010) apresentam diversas razões em favor do uso de funções de ligação assimétricas, principalmente, quando a probabilidade de sucesso aproxima-se de zero numa taxa diferente da qual aproxima-se de um. Chen (2004) empreendeu um estudo de simulação para investigar a importância da escolha da função de ligação na predição da variável resposta em modelos binários univariados. O autor considerou dois esquemas de simulação: (i) os dados são gerados de acordo com o modelo probito; e (ii) os dados são gerados de acordo com o modelo complemento loglog (cloglog). Em ambas as situações, foram ajustados os modelos probito, logito e complemento loglog. O autor observou que, quando a função de ligação verdadeira é a probito, quase não há diferença entre o modelo probito e logito. No entanto, o modelo complemento loglog mostrou-se inadequado. Por outro lado, quando a função de ligação verdadeira é a complemento loglog, os modelos simétricos foram claramente inadequados. O autor concluiu neste estudo empírico que a escolha da função de ligação é muito importante e, caso seja mal especificada, pode fornecer predições pobres.

Para ilustrar o fato de que modelos assimétricos podem apresentar melhor ajuste a um conjunto de dados de que modelos simétricos, nós utilizaremos um estudo bastante simples e conhecido na literatura que mostra claramente que um modelo assimétrico é preferível. Este conjunto de dados é apresentado inicialmente em Bliss (1935) e refere-se ao número de insetos adultos mortos após 5 horas de exposição ao gás carbônico para várias concentrações deste gás. Este conjunto de dados foi estudado por vários autores que propuseram funções de ligação assimétricas em regressão binária, dentre eles, citamos Prentice (1976), Stukel (1988) e Bázan, Branco e Bolfarine (2005). Todos estes autores concluíram que modelos assimétricos são mais convenientes que modelos simétricos para o ajuste neste caso. As proporções de insetos mortos e as curvas das funções de ligação probito e complemento $\log \log (\operatorname{cog} \log )$ ajustadas são apresentadas na Figura 1.1. Note, na Figura 1.1, que o modelo assimétrico utilizado, o modelo c-loglog, aparenta fornecer um melhor ajuste que o simétrico, o modelo probito.

Funções de ligação assimétricas são mais flexíveis que funções de ligação simétricas, o que as tornam bastante úteis, principalmente, quando estas funções de ligação tem como casos especiais funções de ligação simétricas conhecidas. Chen, Dey e Shao (1999) propuseram uma função de ligação assimétrica para modelos com respostas binárias que tem como casos particulares as função probito, t-Student. Bázan, Branco e Bolfarine (2005) apresentaram uma alternativa para o modelo probito assimétrico proposto por Chen, Dey e Shao (1999). A relação entre estes dois modelos probito assimétrico é discutido em detalhes por Bázan, Bolfanine e Branco (2010). Estes autores mencionam que modelos com funções de ligação assimétricas são mais atrativas em diversos aspectos.

Neste trabalho, nós estudaremos as possibilidades de usar modelos com funções de ligação multivariadas para ajustar dados longitudinais ou dados com alguma estrutura de dependência entre as variáveis respostas binárias. O objetivo é estender alguns resultados existentes na literatura 


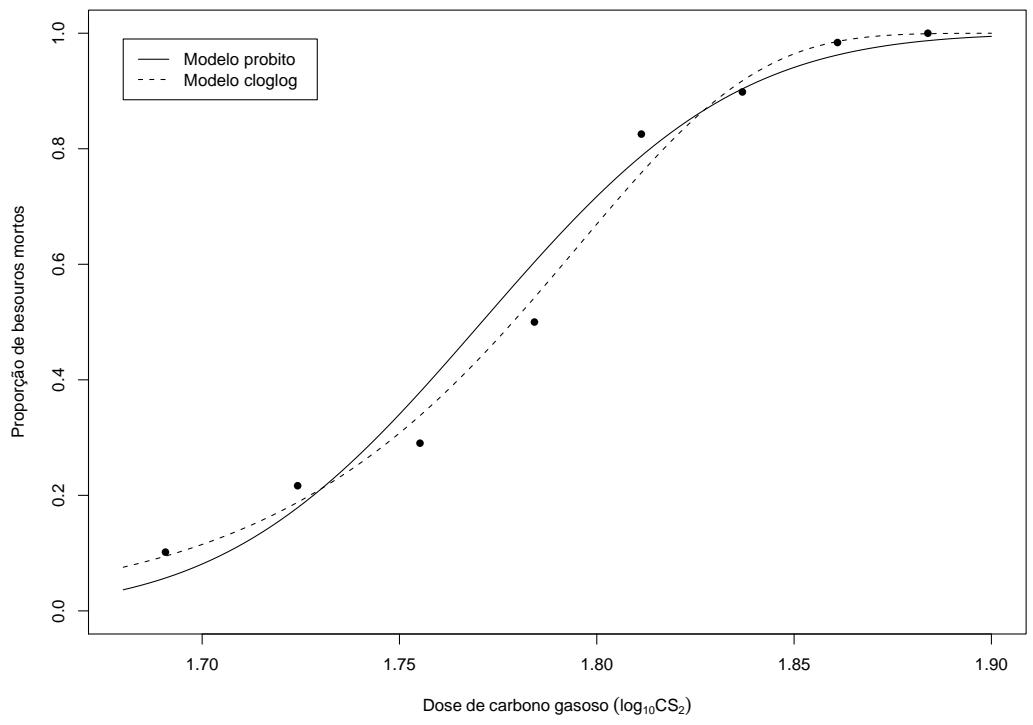

Figura 1.1: Modelos probito e cloglog ajustados à proporção de besouros mortos.

para modelos multivariados simétricos, por exemplo, probito e logito, para modelos com funções de ligação multivariadas assimétricas, tais como modelo multivariado probito assimétrico (MMPA) e o modelo multivariado logístico assimétrico (MMLA). A abordagem utilizada para realizações de inferências é a abordagem bayesiana.

\subsection{Organização do Trabalho}

Esta tese introduz o modelo de regressão multivariado binário com função de ligação multivariada assimétrica. No segundo capítulo introduzimos um modelo de regressão multivariado geral para o ajuste de conjuntos de dados com repostas binárias e apresentamos uma revisão do modelo binário com função de ligação elíptica e propomos uma função de ligação multivariada assimétrica que tem como caso particular as funções de ligação multivariadas simétricas propostas por Chib e Greenberg (1998) e Chen e Dey (2003).

No terceiro capítulo apresentamos algumas abordagens inferênciais para o modelo multivariado binário com função de ligação elíptica assimétrica. Este capítulo apresenta um estudo de identificabilidade para o modelo multivariado binário e obtem as distribuições a posteriori necessárias para a implementação de algoritmos de Monte Carlo via Cadeias de Markov (MCMC), em particular o algoritmo de Gibbs.

Um estudo de simulação é apresentado no quarto capítulo. O estudo de simulação contempla dois objetivos principais. O primeiro objetivo é verificar se os algoritmos apresentados no terceiro capítulo estimam corretamente (recuperam) os valores simulados dos parâmetros à medida que o tamanho da amostra cresce. O segundo objetivo é avaliar qual o impacto nos resultados ao se especificar (erroneamente) uma função de ligação simétrica quando a verdadeira função de ligação é assimétrica. 
No quinto capítulo aplicamos o modelo proposto em um conjunto de dados real bastante conhecido na literatura de modelos multivariados binários. Finalmente, no sexto capítulo apresentamos e discutimos algumas conclusões obtidas na tese. 


\section{Capítulo 2}

\section{Modelos Multivariados Binários}

Considere $Y_{i j}$ uma variável aleatória binária que corresponde a resposta do $i$-ésimo indivíduo para a $j$-ésima variável, e seja $\boldsymbol{Y}_{i}=\left(Y_{i 1}, Y_{i 2}, \ldots, Y_{i J}\right)^{\prime}(i=1, \ldots, n)$ o vetor aleatório com todas as $J$ respostas associadas ao $i$-ésimo indivíduo. Considere também que a cada variável resposta $Y_{i J}$ está associado um vetor $\boldsymbol{x}_{i j}=\left(x_{i j 1}, \ldots, x_{i j k_{j}}\right)^{\prime}$ de variáveis explicativas fixas de dimensão $k_{j}$ e um vetor $\boldsymbol{\beta}_{j}=\left(\boldsymbol{\beta}_{j 1}, \boldsymbol{\beta}_{j 2}, \ldots, \boldsymbol{\beta}_{j k_{j}}\right)^{\prime}$ de coeficientes desconhecidos da regressão. Assumindo que para cada indivíduo $i$, as variáveis aleatórias $Y_{i 1}, Y_{i 2}, \ldots, Y_{i J}$ são correlacionados e os vetores $\boldsymbol{Y}_{1}, \boldsymbol{Y}_{2}, \ldots, \boldsymbol{Y}_{n}$ são independentes, um modelo de regressão multivariado para respostas binárias pode ser expresso da seguinte forma

$$
P\left(Y_{i 1}=y_{i 1}, Y_{i 2}=y_{i 2}, \cdots, Y_{i J}=y_{i J} \mid \boldsymbol{X}_{i}, \boldsymbol{\beta}\right)=\int_{A_{i J}} \cdots \int_{A_{i 1}} f_{J}\left(t_{i 1}, \cdots, t_{i J}\right) d t_{i 1} \cdots d t_{i J}
$$

para $i=1, \ldots n$ e $j=1, \ldots J$, em que $y_{i j}$ é o valor observado da variável $Y_{i j} \in\{0,1\}, f_{J}$ é uma função de densidade de probabilidade (fdp) de uma distribuição $J$-variada e os intervalos de integração $A_{i j}$ são

$$
A_{i j}= \begin{cases}\left(-\infty, \boldsymbol{x}_{i j}^{\prime} \boldsymbol{\beta}_{j}\right] & \text { se } y_{i j}=1 \\ \left(\boldsymbol{x}_{i j}^{\prime} \boldsymbol{\beta}_{j},+\infty\right) & \text { se } y_{i j}=0\end{cases}
$$

$\operatorname{com} \boldsymbol{\beta}=\left(\boldsymbol{\beta}_{1}, \boldsymbol{\beta}_{2}, \ldots, \boldsymbol{\beta}_{J}\right)^{\prime}$ denotando o vetor com todos os $k=\sum_{j=1}^{J} k_{j}$ coeficientes regressores e

$$
\boldsymbol{X}_{i}=\left(\begin{array}{ccccc}
\boldsymbol{x}_{i 1}^{\prime} & 0 & 0 & \cdots & 0 \\
0 & \boldsymbol{x}_{i 2}^{\prime} & 0 & \cdots & 0 \\
\vdots & \cdots & \ddots & \ldots & \vdots \\
0 & \cdots & 0 & \boldsymbol{x}_{i, J-1}^{\prime} & 0 \\
0 & \cdots & 0 & 0 & \boldsymbol{x}_{i J}^{\prime}
\end{array}\right)
$$

denotando a matriz $J \times k$ de variáveis explicativas (covariáveis) associada ao $i$-ésimo indivíduo. Note que cada intervalo definido em (2.2) é determinado por seu próprio conjunto de covariáveis $\boldsymbol{x}_{i j}$ e de regressores $\boldsymbol{\beta}_{j}$. Observe que a função de densidade de probabilidade $f_{J}$ define completamente a forma da relação entre as variáveis explicativas e as probabilidades de interesse. A função de 
verossimilhança deste modelo é dada por

$$
L(\boldsymbol{\beta} ; \boldsymbol{y}, \boldsymbol{X})=\prod_{i=1}^{n} P\left(\boldsymbol{Y}_{i 1}=\boldsymbol{y}_{i 1}, \mid \boldsymbol{X}_{i}, \boldsymbol{\beta}\right)=\prod_{i=1}^{n} \int_{A_{i J}} \ldots \int_{A_{i 1}} f_{J}\left(t_{i 1}, \cdots, t_{i J}\right) d t_{i 1} \cdots d t_{i J} .
$$

É importante notar que esta verossimilhança pode depender de outros parâmetros desconhecidos além do vetor $\boldsymbol{\beta}$, por exemplo, parâmetros de forma e de dependência. Estes parâmetros adicionais, caso existam, são especificados pela fdp $f_{J}$. O fato da função de verossimilhança (2.4) depender de integrais múltiplas pode prejudicar a eficiência dos algoritmos de estimação. Seja pelo método de máxima verossimilhança ou na obtenção das distribuições a posteriori para o uso do Monte Carlo via Cadeia de Markov (MCMC).

Albert e Chib (1993) observaram que o modelo de regressão binária pode ser especificado em termos de uma variável aleatória latente normalmente distribuída. Esta formulação alternativa facilita na implementação de algoritmos MCMC, o que torna o modelo computacionalmente mais atrativo para o uso de inferência bayesiana e na implementação do algoritmo EM (Dempster, Laird e Rubin, 1977) na abordagem frequentista. Albert e Chib (1993) discutiram esta abordagem para os modelos probito e t-Student. Mais recentemente, Chib e Greenberg (1998) consideraram esta abordagem nos modelos multivariados probito (MMP) e Chen e Dey (2003) nos modelos multivariados probito (MMP), $t$-Student (MMT) e logístico (MML).

Para a especificação do modelo definido em (2.1) através de variáveis latentes, considere $\boldsymbol{Z}_{i}=$ $\left(Z_{i 1}, \ldots, Z_{i J}\right)^{\prime}$ um vetor aleatório $J$-dimensional de variáveis latentes tal que

$$
\boldsymbol{Z}_{i}=\boldsymbol{X}_{i} \boldsymbol{\beta}-\boldsymbol{\epsilon}_{i}, \operatorname{com} \boldsymbol{\epsilon}_{i} \sim F_{J}, i=1, \ldots, n,
$$

em que $F_{J}$ é a função de distribuição acumulada (fda) que define a função de ligação utilizada no modelo multivariado binário apresentado em (2.1). Temos, condicionando o valor de $Y_{i j}$ de acordo com o sinal de $Z_{i j}$, que o modelo de regressão multivariada com resposta binária definido em (2.1) pode ser representado, equivalentemente, através de variáveis latentes da seguinte forma:

$$
Y_{i j}= \begin{cases}1 & \text { se } Z_{i j}>0 \\ 0 & \text { se } Z_{i j} \leq 0\end{cases}
$$

com

$$
\begin{aligned}
\boldsymbol{Z}_{i} & =\boldsymbol{X}_{i} \boldsymbol{\beta}-\boldsymbol{\epsilon}_{i} \\
\boldsymbol{\epsilon}_{i} & \sim F_{J} .
\end{aligned}
$$

em que $\boldsymbol{\epsilon}_{i}, i=1, \ldots, n$ são vetores aleatórios independentes.

O modelo multivariado probito introduzido por Chib e Greenberg (1998) é um caso particular do modelo multivariado binário definido em (2.6)-(2.8) que ocorre quando os vetores aleatórios $J$ variados $\boldsymbol{\epsilon}_{i}^{\prime} s$, são independentes e normalmente distribuídos. A equivalência entre os modelos (2.1) 
e (2.6)-(2.8) é demonstrada adiante, inicialmente apresentada para o caso marginal (univariado), e depois estendida para o caso multivariado.

Note que o valor da variável $Y_{i j}$ no modelo (2.6)-(2.8) é completamente determinado pelo valor da variável latente $Z_{i j}$ e que as probabilidades dos eventos $Y_{i j}=1$ e $Y_{i j}=0$ são dadas, respectivamente, por

$$
P\left(Y_{i j}=1 \mid \boldsymbol{x}_{i}, \boldsymbol{\beta}_{j}\right)=P\left(Z_{i j}>0 \mid \boldsymbol{x}_{i j} \boldsymbol{\beta}_{j}\right) \text { e } P\left(Y_{i j}=0 \mid \boldsymbol{x}_{i j}, \boldsymbol{\beta}_{j}\right)=P\left(Z_{i j} \leq 0 \mid \boldsymbol{x}_{i j}, \boldsymbol{\beta}_{j}\right) .
$$

Estas duas probabilidades podem ser escritas em uma só expressão da seguinte forma

$$
P\left(Y_{i j}=y_{i j} \mid \boldsymbol{x}_{i j}, \boldsymbol{\beta}_{j}\right)=P\left(Z_{i j} \in B_{i j} \mid \boldsymbol{x}_{i j}, \boldsymbol{\beta}_{j}\right)
$$

em que

$$
B_{i j}=\left\{\begin{array}{cc}
(0, \infty) & \text { se } y_{i j}=1 \\
(-\infty, 0] & \text { se } y_{i j}=0
\end{array}\right.
$$

para $i=1, \ldots, n$ e $j=1, \ldots, J$. Assumindo agora que $Z_{i j}=\boldsymbol{x}_{i j}^{\prime} \boldsymbol{\beta}_{j}-\epsilon_{i j}$ e $\epsilon_{i j} \sim F_{1 j}$, em que $F_{1 j}$ é a $j$-ésima distribuição marginal da distribuição conjunta $F_{J}$ definida em (2.8), temos que

$$
P\left(Y_{i j}=1 \mid \boldsymbol{x}_{i j}, \boldsymbol{\beta}_{j}\right)=P\left(Z_{i j}>0 \mid \boldsymbol{x}_{i j}, \boldsymbol{\beta}_{j}\right)=P\left(\boldsymbol{x}_{i j}^{\prime} \boldsymbol{\beta}_{j}-\epsilon_{i j}>0\right)=P\left(\epsilon_{i j}<\boldsymbol{x}_{i j}^{\prime} \boldsymbol{\beta}_{j}\right)=\int_{-\infty}^{\boldsymbol{x}_{i j}^{\prime} \boldsymbol{\beta}_{j}} f_{1 j}(t) d t
$$

e

$$
P\left(Y_{i j}=0 \mid \boldsymbol{x}_{i j}, \boldsymbol{\beta}_{j}\right)=P\left(Z_{i j} \leq 0 \mid \boldsymbol{x}_{i j}, \boldsymbol{\beta}_{j}\right)=P\left(\boldsymbol{x}_{i j}^{\prime} \boldsymbol{\beta}_{j}-\epsilon_{i j} \leq 0\right)=P\left(\epsilon_{i j} \geq \boldsymbol{X}_{i} \boldsymbol{\beta}\right)=\int_{\boldsymbol{x}_{i j}^{\prime} \boldsymbol{\beta}_{j}}^{\infty} f_{1 j}(t) d t
$$

que pode ser escrita como uma só expressão da seguinte forma:

$$
P\left(Y_{i j}=y_{i j} \mid \boldsymbol{x}_{i j}, \boldsymbol{\beta}_{j}\right)=\int_{A_{i j}} f_{1 j}(t) d t
$$

com

$$
A_{i j}= \begin{cases}\left(-\infty, \boldsymbol{x}_{i j}^{\prime} \boldsymbol{\beta}_{j}\right] & \text { se } y_{i j}=1 \\ \left(\boldsymbol{x}_{i j}^{\prime} \boldsymbol{\beta}_{j},+\infty\right) & \text { se } y_{i j}=0 .\end{cases}
$$

e a equivalência entre os modelos (2.1) e (2.6)-(2.8) está demonstrada para o caso marginal.

Estendendo agora (2.9) para caso multivariado, temos que

$$
P\left(Y_{i 1}=y_{i 1}, \cdots, Y_{i J}=y_{i J} \mid \boldsymbol{X}_{i}, \boldsymbol{\beta}\right)=P\left(Z_{i 1} \in B_{i 1}, \cdots, Z_{i J} \in B_{i J} \mid \boldsymbol{X}_{i}, \boldsymbol{\beta}\right),
$$

em que os intervalos $B_{i j}(i=1, \ldots n ; j=1, \ldots J)$, são definidos como

$$
B_{i j}=\left\{\begin{array}{cc}
(0, \infty) & \text { se } y_{i j}=1 \\
(-\infty, 0] & \text { se } y_{i j}=0
\end{array}\right.
$$


Combinando aqui (2.10) com a espressão (2.7) temos

$$
Z_{i j}=\boldsymbol{x}_{i j}^{\prime} \boldsymbol{\beta}_{j}-\epsilon_{i j} \in B_{i j}
$$

para $i=1, \ldots n$ e $j=1, \ldots J$. Após algumas operações com os intervalos $B_{i j}^{\prime}$ s e produtos $\boldsymbol{x}_{i j}^{\prime} \boldsymbol{\beta}_{j}$, temos que o modelo (2.10) e a expressão (2.11) somente são satisfeitos se a variável aleatória $\epsilon_{i j}$ pertence ao intervalo

$$
A_{i j}= \begin{cases}\left(-\infty, \boldsymbol{x}_{i j}^{\prime} \boldsymbol{\beta}_{j}\right] & \text { se } y_{i j}=1 \\ \left(\boldsymbol{x}_{i j}^{\prime} \boldsymbol{\beta}_{j},+\infty\right) & \text { se } y_{i j}=0\end{cases}
$$

e, portanto, temos que o modelo multivariado binário (2.6)-(2.8) pode ser escrito da seguinte forma

$$
\begin{aligned}
P\left(Y_{i 1}=y_{i 1}, \cdots, Y_{i J}=y_{i J} \mid \boldsymbol{X}_{i}, \boldsymbol{\beta}\right) & =P\left(\epsilon_{1} \in A_{i 1}, \cdots, \epsilon_{J} \in A_{i J} \mid \boldsymbol{X}_{i}, \boldsymbol{\beta}\right) \\
& =\int_{A_{i J}} \cdots \int_{A_{i 1}} f_{J}\left(t_{i 1}, \cdots, t_{i J}\right) d t_{i 1} \cdots d t_{i J}
\end{aligned}
$$

Que é o mesmo modelo multivariado binário apresentado em (2.1). Logo, a equivalência entre os modelos (2.1) e (2.6)-(2.8) está demonstrada.

Note que até o momento não foi discutido qual será a estrutura de dependência assuminda entre as variáveis respostas binárias neste trabalho. Contudo, ao se considerar o modelo multivariado probito definido por Chib e Greenberg (1998), estamos assumindo que a estrutura de correlação entre as variável resposta é definida e modelada através da variável latente que especifica a função de ligação utilizada. A escolha dstas variáveis latentes determinam, conseqüentemente, uma estrutura de dependência entre as variáveis respostas binárias. Chib (2000) argumenta que modelar a estrutura de dependência entre as variáveis binárias através da distribuição da variável latente é bastante flexível e geral. O autor argumenta que modelar as correlações diretamente através de razões de chances marginais leva a dificuldades, seja ela causada pela dificuldade em especificar uma estrutura geral de correlações para todos os pares de respostas, ou simplesmente por não ser natural pensar em correlação entre variáveis respostas binárias.

Com o fim de especificar completamente o modelo de regressão binária, as próximas seções apresentam classes de distribuições multivariadas que podem ser utilizadas como funções de ligação para os modelos multivariados com respostas binárias.

\subsection{Modelo multivariado com função de ligação elíptica}

A classe de distribuições elípticas (Fang, Kotz e Ng, 1990) representam alternativas simétricas para a classe de distribuições normais. De fato, a distribuição normal é um caso particular da família de distribuições elípticas. Além da distribuição normal, a classe de distribuições elípticas contempla as distribuições $t$-Student, logística, exponencial potência e Cauchy, dentre outras. Estas distribuições elípticas ocupam hoje um papel importante na teoria estatística já que é uma classe mais robusta, pois supõem que os dados podem ter caudas mais leves ou mais pesadas que a distribuição normal. Recomendamos Fang, Kotz e Ng (1990), Fang e Anderson (1990) e Arellano-Valle (1994) 
como uma fonte de pesquisa sobre distribuições simétricas e elípticas. Este autores apresentam suas principais propriedades e aspectos inferenciais.

No contexto de regressão binária, esta flexibilidade nas caudas leva a funções de ligações mais flexíveis, acolhendo dados em que as probabilidades de sucesso crescem (decrescem) numa taxa diferente daquela especificada pela distribuição normal e, consequentemente, pelo modelo probito.

Ao longo do texto é considerada a seguinte definição de distribuição elíptica multivariada.

Definição 1. Um vetor aleatório $\boldsymbol{Z} \in \mathbb{R}^{J}$ segue uma distribuiçâao de contornos elípticos (elíptica), denotada por $\boldsymbol{Z} \sim E l_{J}\left(\boldsymbol{\mu}, \boldsymbol{\Sigma}, g^{(J)}\right)$, se sua função de densidade de probabilidade (fdp) tem a forma

$$
f_{E l}\left(\boldsymbol{z} \mid \boldsymbol{\mu}, \boldsymbol{\Sigma}, g^{(J)}\right)=|\boldsymbol{\Sigma}|^{-\frac{1}{2}} g\left[(\boldsymbol{z}-\boldsymbol{\mu})^{\prime} \boldsymbol{\Sigma}^{-1}(\boldsymbol{z}-\boldsymbol{\mu})\right]
$$

em que $\boldsymbol{\mu} \in \mathbb{R}^{J}$ é um vetor de parâmetros de localização, $\boldsymbol{\Sigma} \in \mathbb{R}^{J \times J}$ é uma matriz de escala (matriz simétrica e positiva definida) e $g^{(J)}: \mathbb{R}_{+} \rightarrow \mathbb{R}_{+}$é uma função contínua e diferenciável chamada de função geradora de densidades, a qual satisfaz a condição

$$
g^{(J)}(u)=\frac{\Gamma(J / 2)}{\pi^{J / 2}} \frac{g(u ; J)}{\int_{0}^{\infty} u^{J / 2-1} g(u ; J) d u},
$$

com $g(u ; J)$ uma função dos $\mathbb{R}_{+}$nos $\mathbb{R}_{+}$tal que $\int_{0}^{\infty} u^{J / 2-1} g(u ; J) d u$ existe.

A função geradora de densidades $g^{(J)}$ definida em (2.12) pode depender de outros parâmetros. Por exemplo, no caso da distribuição $t$-Student, o parâmetro adicional é o grau de liberdade. A Tabela 2.1 apresenta a forma da função geradora de densidades para algumas distribuições elípticas.

Tabela 2.1: Função geradora de densidade de algumas distribuições elípticas.

\begin{tabular}{lll}
\hline Distribuição & Notação & Função geradora de densidades \\
\hline Normal & $\mathcal{N}_{J}(\boldsymbol{\mu}, \boldsymbol{\Sigma})$ & $g^{(J)}(u)=(2 \pi)^{J / 2} \exp (-u / 2)$ \\
t-Student & $S t_{J}(\boldsymbol{\mu}, \boldsymbol{\Sigma}, \nu)$ & $g^{(J)}(u)=c_{1}\left(1+\frac{u}{\nu}\right)^{-(\nu+J) / 2}$ \\
t-Student generalizada & $G S t_{J}(\boldsymbol{\mu}, \boldsymbol{\Sigma}, \nu, \gamma)$ & $g^{(J)}(u)=c_{2}\left(1+\frac{u}{\gamma}\right)^{-(\nu+J) / 2}$ \\
Exponencial potência & $E P_{J}(\boldsymbol{\mu}, \boldsymbol{\Sigma}, \lambda)$ & $g^{(J)}(u)=c_{3} \exp \left(-u^{\lambda} / 2\right)$ \\
Cauchy & $C_{J}(\boldsymbol{\mu}, \boldsymbol{\Sigma})$ & $g^{(J)}(u)=c_{4}(1+u)^{-(J+1) / 2}$ \\
Logística & $L_{J}(\boldsymbol{\mu}, \boldsymbol{\Sigma})$ & $g^{(J)}(u)=c_{5} \exp (-u) /\{1+\exp (-u)\}^{2}$ \\
\hline
\end{tabular}

em que $c_{1}, c_{2}, c_{3}$, e $c_{4}$ são constantes normalizadoras apropriadas. Note que as distribuições $\mathrm{t}$-Student e Cauchy são casos particulares da distribuição t-Student generalizada quando $\gamma=\nu \mathrm{e}$ $\gamma=\nu=1$, respectivamente.

Considere agora que o vetor de variáveis latentes $\boldsymbol{Z}_{i}$ definido no modelo de regressão binário (2.6)-(2.8) segue uma distribuição elíptica $J$-variada com função de densidade de probabilidade dada em (2.12). Temos, neste caso, que o modelo multivariado binário com função de ligação elíptica 
é dado por

$$
P\left(\boldsymbol{Y}_{i}=\boldsymbol{y}_{i} \mid \boldsymbol{X}_{i}, \boldsymbol{\beta}\right)=\int_{A_{i J}} \ldots \int_{A_{i 1}}|\boldsymbol{\Sigma}|^{-\frac{1}{2}} g\left(\boldsymbol{z}_{i}^{\prime} \boldsymbol{\Sigma}^{-1} \boldsymbol{z}_{i}\right) d \boldsymbol{z}_{i},
$$

em que $g$ a função geradora de densidades definida em (2.13) e

$$
A_{i j}= \begin{cases}\left(-\infty, \boldsymbol{x}_{i j}^{\prime} \boldsymbol{\beta}_{j}\right] & \text { se } y_{i j}=1 \\ \left(\boldsymbol{x}_{i j}^{\prime} \boldsymbol{\beta}_{j},+\infty\right) & \text { se } y_{i j}=0 .\end{cases}
$$

Observe que o intervalo $A_{i j}$ é função dos vetor de parâmetros $\boldsymbol{\beta}_{j}$. Isto pode tornar o processo inferencial um pouco mais complicado. No entanto, o modelo binário definido em (2.5) apresenta a propriedade de aditividade entre os termos $\boldsymbol{X}_{i} \boldsymbol{\beta}$ e $\boldsymbol{\epsilon}_{i}$. Esta propriedade nos permite escrever o modelo multivariado binário com função de ligação elíptica da seguinte forma

$$
P\left(\boldsymbol{Y}_{i}=\boldsymbol{y}_{i} \mid \boldsymbol{X}_{i}, \boldsymbol{\beta}\right)=\int_{B_{i J}} \ldots \int_{B_{i 1}}|\boldsymbol{\Sigma}|^{-\frac{1}{2}} g\left[\left(\boldsymbol{z}_{i}-\boldsymbol{X}_{i} \boldsymbol{\beta}\right)^{\prime} \boldsymbol{\Sigma}^{-1}\left(\boldsymbol{z}_{i}-\boldsymbol{X}_{i} \boldsymbol{\beta}\right)\right] d \boldsymbol{z}_{i},
$$

em que $B_{i j}$ é dado por

$$
B_{i j}=\left\{\begin{array}{cc}
(0, \infty) & \text { se } y_{i j}=1 \\
(-\infty, 0] & \text { se } y_{i j}=0
\end{array}\right.
$$

Consequentemente, a função de verossimilhança é definida como

$$
L(\boldsymbol{\beta}, \boldsymbol{\Sigma} ; \boldsymbol{y}, \boldsymbol{X})=\prod_{i=1}^{n} \int_{B_{i J}} \ldots \int_{B_{i 1}}|\boldsymbol{\Sigma}|^{-\frac{1}{2}} g\left[\left(\boldsymbol{z}_{i}-\boldsymbol{X}_{i} \boldsymbol{\beta}\right)^{\prime} \boldsymbol{\Sigma}^{-1}\left(\boldsymbol{z}_{i}-\boldsymbol{X}_{i} \boldsymbol{\beta}\right)\right] d \boldsymbol{z}_{i} .
$$

O fato da função de verossimilhança definida (2.16) depender de integrais múltiplas pode prejudicar a eficiência dos algoritmos de estimação. Seja pelo método de máxima verossimilhança ou na obtenção das distribuições a posteriori para o uso do MCMC. A abordagem via dados aumentados para modelos binários proposta por Albert e Chib (1993) é uma alternativa de modelagem que pode ser utilizada para evitar a necessidade de trabalhar com integrais múltiplas presente na função de verossimilhança (2.16). Esta abordagem é natural em nosso caso, pois o modelo multivariado binário com função de ligação elíptica definido em (2.14) foi induzido pelos vetores de variáveis latentes $\boldsymbol{Z}_{1}, \boldsymbol{Z}_{2}, \ldots, \boldsymbol{Z}_{n}$.

A função de verossimilhança aumentada (pelos dados latentes) do modelo multivariado binário com função de ligação elíptica é

$$
L_{D A}(\boldsymbol{\beta}, \boldsymbol{\Sigma} ; \boldsymbol{z}, \boldsymbol{y}, \boldsymbol{X})=\prod_{i=1}^{n}|\boldsymbol{\Sigma}|^{-\frac{1}{2}} g\left[\left(\boldsymbol{z}_{i}-\boldsymbol{X}_{i} \boldsymbol{\beta}\right)^{\prime} \boldsymbol{\Sigma}^{-1}\left(\boldsymbol{z}_{i}-\boldsymbol{X}_{i} \boldsymbol{\beta}\right)\right] I\left(\boldsymbol{z}_{i} \in B_{i}\right),
$$

em que $B_{i}=B_{i 1} \times B_{i 2} \times \ldots B_{i J}$, com

$$
B_{i j}= \begin{cases}(0,+\infty) & \text { se } y_{i j}=1 \\ (-\infty, 0] & \text { se } y_{i j}=0\end{cases}
$$


Os dados aumentados facilitam a implementação computacional dos algoritmos de estimação dos parâmetros de interesse nos modelos binários com funções de ligação elíptica. Isto ocorre, principalmente, com as distribuições elípticas pertencentes à subclasse de distribuições representáveis através de misturas no parâmetro de escala da distribuição normal (Andrews e Mallows, 1974).

Definição 2. Um vetor aleatório $\boldsymbol{Z} \in \mathbb{R}^{J}$ segue uma distribuição representável através de misturas no parâmetro de escala da distribuição normal, denotado por $M E N(\boldsymbol{\mu}, \boldsymbol{\Sigma}, H)$, se sua função de densidade de probabilidade (fdp) pode ser escrita como

$$
f(\boldsymbol{z} \mid \boldsymbol{\mu}, \boldsymbol{\Sigma}, H)=\int_{0}^{\infty} \phi_{J}(\boldsymbol{z} \mid \boldsymbol{\mu}, \kappa(\eta) \boldsymbol{\Sigma}) d H(\eta),
$$

em que $\phi_{J}(\boldsymbol{z} \mid \boldsymbol{\mu}, \boldsymbol{\Sigma})$ denota a função de densidade de probabilidade de uma distribuição normal $J$-variada com vetor de parâmetros de localizaçâo $\boldsymbol{\mu} \in \mathbb{R}^{J}$ e matriz de escala $\boldsymbol{\Sigma} \in \mathbb{R}^{J \times J}$ (matriz simétrica e positiva definida). A função $H$ é uma função de distribuição probabilidade unidimensional e $\kappa$ é uma função unidimensional estritamente positiva.

A Mistura de Escala Normal (MEN) é uma classe ampla que contempla muitas distribuições unimodais e simétricas, tais como as famílias $t$-Student, logística, laplace, exponencial potência, stable e slash. As propriedades das MEN tem sido úteis em diversas áreas (Andrews e Mallows, 1974; Chen e Dey, 1998, 2000; West, 1987). A classe de mistura de escala normal (MEN) foi primeiramente utilizada na construção de funções de ligação para modelos multivariados binários por Chen e Dey (1998).

Partindo da função de verossimilhança aumentada dada em (2.17), temos que a função de verossimilhança aumentada nos modelos binários com funções de ligação pertencentes a subclasse de distribuições obtidas através de misturas no parâmetro de escala da distribuição normal é dada por

$$
L_{D A}(\boldsymbol{\beta}, \boldsymbol{\Sigma} ; \boldsymbol{z}, \boldsymbol{y}, \boldsymbol{X})=\prod_{i=1}^{n} \int_{0}^{\infty} \phi_{J}\left(\boldsymbol{z}_{i} \mid \boldsymbol{X}_{i} \boldsymbol{\beta} ; \kappa(\eta) \boldsymbol{\Sigma}\right) d H(\eta) I\left(\boldsymbol{z}_{i} \in B_{i}\right),
$$

em que $\kappa(\eta)$ e $H(\eta)$ são as mesmas funções dadas na Definição 2. Utilizando a seguinte representação das distribuições MEN,

$$
\boldsymbol{z} \mid \eta \sim \mathcal{N}(\boldsymbol{\mu}, \kappa(\eta) \boldsymbol{\Sigma}) \text { e } \eta \sim H
$$

temos que a função de verossimilhança (2.18) pode ser novamente aumentada usando a variável de mistura $\eta$ da seguinte forma

$$
L_{D A}(\boldsymbol{\beta}, \boldsymbol{\Sigma} ; \boldsymbol{\eta}, \boldsymbol{z}, \boldsymbol{y}, \boldsymbol{X})=\prod_{i=1}^{n} \phi_{J}\left(\boldsymbol{z}_{i} \mid \boldsymbol{X}_{i} \boldsymbol{\beta} ; \kappa\left(\eta_{i}\right) \boldsymbol{\Sigma}\right) h\left(\eta_{i}\right) I\left(\boldsymbol{z}_{i} \in B_{i}\right),
$$

em que $h$ é a função densidade de probabilidade associada a função de distribuição $H$. Um exemplo típico de uma distribuição pertencente a esta classe é a distribuição t-Student, a qual é obtida quando $\kappa(\eta)=1 / \eta$ e $\eta$ segue uma distribuição gamma. Os modelos MEN são bastante robustos a observações discrepantes. No entanto, não comportam assimetria na função de ligação. A assimetria na função de ligação ocorre quando a probabilidade de sucesso cresce em uma taxa diferente da taxa que decresce. Na próxima seção é introduzida uma classe maior e mais flexível de distribuições que podem ser utilizadas como funções de ligação para modelos multivariados binários. 


\subsection{Modelo multivariado com função de ligação elíptica assimétrica}

Existe uma variedade grande de possibilidades para a construção de distribuições elípticas assimétricas no contexto multivariado e, em geral, estas diferentes abordagens podem nos levar a diferentes classes de distribuições. Azzalini e Dalla-Valle (1996) estenderam para o caso multivariado a distribuição normal assimétrica proposta por Azzalini (1985). Mais recentemente Branco e Dey (2001) estenderam via condicionamento os resultados da distribuição normal assimetrica introduzida por Azzalini e Dalla-Valle (1996) para obter distribuições elípticas assimétricas. Uma ampla revisão sobre as diferentes distribuições elípticas assimétricas, suas propriedades e aplicações, é apresentada em Genton (2004).

A classe de distribuições multivariadas elípticas assimétricas considerada nesta tese contempla as distribuições pertencentes à classe de distribuições assimétricas propostas por Sahu, Dey e Branco (2003). Esta classe permite uma representação estocástica que facilita a implementação dos algoritmos de estimação. A definição é dada a seguir.

Definição 3. Um vetor aleatório $\boldsymbol{Z} \in \mathbb{R}^{J}$ segue uma distribuição elíptica assimétrica, denotada por $S E_{J}\left(\boldsymbol{\mu}, \boldsymbol{\Sigma}, \boldsymbol{D}, g^{(J)}\right)$, se sua fdp tem a forma

$$
\begin{aligned}
& f_{S E}\left(\boldsymbol{z} \mid \boldsymbol{\mu}, \boldsymbol{\Sigma}, \boldsymbol{D}, g^{(J)}\right)=2^{J} f_{E l}\left(\boldsymbol{z} \mid \boldsymbol{\mu}, \boldsymbol{\Sigma}+\boldsymbol{D}^{2}, g^{(J)}\right) \times \\
& \quad F_{E l}\left(\boldsymbol{D}\left(\boldsymbol{\Sigma}+\boldsymbol{D}^{2}\right)^{-1}(\boldsymbol{z}-\boldsymbol{\mu}) \mid \mathbf{0}, \boldsymbol{I}-\boldsymbol{D}\left(\boldsymbol{\Sigma}+\boldsymbol{D}^{2}\right)^{-1} \boldsymbol{D}, g_{q(\boldsymbol{z}-\boldsymbol{\mu})}^{(J)}\right)
\end{aligned}
$$

em que $f_{E l}\left(\boldsymbol{z} \mid \boldsymbol{\mu}, \boldsymbol{\Sigma}, g_{q}^{(J)}\right)$ e $F_{E l}\left(\boldsymbol{z} \mid \boldsymbol{\mu}, \boldsymbol{\Sigma}, g_{q}^{(J)}\right)$ são as funçôes de densidade de probabilidade (fdp) e funçẫo densidade acumulada (fda) de uma distribuiçấo elíptica multivariada (ver Definiçẫo 1) com parâmetro de localização $\boldsymbol{\mu}$, matriz de dispersão $\boldsymbol{\Sigma}$, matriz de forma diagonal $\boldsymbol{D}$ e funçẫo geradora de densidade $g_{q}^{(J)}:[0, \infty) \rightarrow[0, \infty)$, em que

$$
q(\boldsymbol{z}-\boldsymbol{\mu})=(\boldsymbol{z}-\boldsymbol{\mu})^{\prime}\left(\boldsymbol{\Sigma}+\boldsymbol{D}^{2}\right)^{-1}(\boldsymbol{z}-\boldsymbol{\mu}) .
$$

define completamente o modelo.

$$
g_{a}^{(J)}(u)=\frac{\Gamma(J / 2)}{\pi^{J / 2}} \frac{g^{(J)}(a+u)}{\int_{0}^{\infty} r^{J / 2-1} g^{(J)}(a+u) d r}, a>0
$$

em que $g(a+u ; 2 J): \mathbb{R}_{+} \rightarrow \mathbb{R}_{+}$é função tal que $\int_{0}^{\infty} r^{J / 2-1} g^{(J)}(a+u) d r$ existe. A função $g^{(J)}(a+u)$ fornece o núcleo da densidade da distribuição elíptica original, a qual pode depender de parâmetros adicionais.

Para a construção de um modelo binário com função de ligação assimétrica, considere que a variável latente $\boldsymbol{Z}_{i}$ definida no modelo de regressão binária (2.6)-(2.8), segue uma distribuição elíptica assimétrica $J$-variada com função densidade de probabilidade dada em (2.19). O modelo multivariado binário com função de ligação assimétrica resultante é da forma

$$
\begin{aligned}
P\left(\boldsymbol{Y}_{i}=\boldsymbol{y}_{i} \mid \boldsymbol{X}_{i}, \boldsymbol{\beta}, \boldsymbol{\Sigma}, \boldsymbol{D}\right)=\int_{A_{i J}} \ldots \int_{A_{i 1}} 2^{J} f_{E l}\left(\boldsymbol{z} \mid \mathbf{0}, \boldsymbol{\Sigma}+\boldsymbol{D}^{2}, g^{(J)}\right) \\
\times F_{E l}\left(\boldsymbol{D}\left(\boldsymbol{\Sigma}+\boldsymbol{D}^{2}\right)^{-1} \boldsymbol{z} \mid \mathbf{0}, \boldsymbol{I}-\boldsymbol{D}\left(\boldsymbol{\Sigma}+\boldsymbol{D}^{2}\right)^{-1} \boldsymbol{D}, g_{q(\boldsymbol{z})}^{(J)}\right) d \boldsymbol{z},
\end{aligned}
$$


com

$$
A_{i j}= \begin{cases}\left(-\infty, \boldsymbol{x}_{i j}^{\prime} \boldsymbol{\beta}_{j}\right] & \text { se } y_{i j}=1 \\ \left(\boldsymbol{x}_{i j}^{\prime} \boldsymbol{\beta}_{j},+\infty\right) & \text { se } y_{i j}=0\end{cases}
$$

Esta classe de distribuições elípticas assimétricas é composta por uma ampla gama de distribuições flexíveis e robustas, pois contempla distribuições simétricas e assimétricas, as quais podem apresentar caudas mais leves ou mais pesadas do que a distribuição normal. Além de comportar tanto assimetria positiva quanto negativa. Casos particulares destas distribuições elípticas assimétricas são as distribuições representáveis através de mistura no parâmetro de escala da distribuição normal assimétrica. Esta subclasse também é bastante flexível e robusta. Ela abrange diversas distribuições elípticas assimétricas, por exemplo, as versões simétricas e assimétricas das distribuições normal, $t$-Student e logística. No entanto, ela apresenta a vantagem de poder ser representada hierarquicamente. A Mistura de Escala Normal Assimétrica (MENA) multivariada foi introduzida em Branco e Dey (2001) e recentemente estudada em Vernic (2005) e Kim (2008). Neste trabalho nós utilizaremos a distribuição normal assimétrica desenvolvida por Sahu, Dey e Branco (2003). Esta representação facilita a construção de algoritmos de estimação.

Considere a seguinte definição de modelos representáveis através de mistura no parâmetro de escala de distribuições normal assimétrica (MENA).

Definição 4. Um vetor aleatório $\boldsymbol{Z} \in \mathbb{R}^{J}$ segue uma distribuição de mistura no parâmetro de escala da distribuição normal assimétrica, denotado por $\operatorname{MENA}(\boldsymbol{\mu}, \boldsymbol{\Sigma}, \boldsymbol{D}, H)$, se sua função de densidade de probabilidade (fdp) pode ser escrita como

$$
\begin{aligned}
f(\boldsymbol{z} \mid \boldsymbol{\mu}, \boldsymbol{\Sigma}, \boldsymbol{D}, H)= & 2^{J} \int_{0}^{\infty} \phi_{J}\left[\boldsymbol{z} \mid \boldsymbol{\mu}, \kappa(\eta)\left(\boldsymbol{\Sigma}+\boldsymbol{D}^{2}\right)\right] \times \\
& \Phi_{J}\left\{\boldsymbol{D}\left(\boldsymbol{\Sigma}+\boldsymbol{D}^{2}\right)^{-1}(\boldsymbol{z}-\boldsymbol{\mu}) \mid \mathbf{0},\left(I+\boldsymbol{D}\left(\boldsymbol{\Sigma}+\boldsymbol{D}^{2}\right)^{-1} \boldsymbol{D}\right) \kappa(\eta)\right\} d H(\eta),
\end{aligned}
$$

em que $\phi_{J}(\boldsymbol{z} \mid \boldsymbol{\mu}, \boldsymbol{\Sigma})$ e $\Phi_{J}(\boldsymbol{z} \mid \boldsymbol{\mu}, \boldsymbol{\Sigma})$ denotam, respectivamente, as funçôes de densidade de probabilidade e acumulada de uma distribuição normal J-variada com vetor de parâmetros de localizaçâa $\boldsymbol{\mu} \in \mathbb{R}^{J}$ e matriz de escala $\boldsymbol{\Sigma} \in \mathbb{R}^{J \times J}$. A função $H$ é uma função de distribuição probabilidade unidimensional e $\kappa$ é uma função unidimensional estritamente positiva.

As distribuições pertencentes à família MENA podem ser representadas estocasticamente da seguinte forma.

$$
\boldsymbol{Z}=\boldsymbol{\mu}+\kappa(\eta)^{1 / 2} \boldsymbol{Z}_{0}
$$

com

$$
\boldsymbol{Z}_{0} \sim \operatorname{SN}_{J}(\mathbf{0}, \boldsymbol{\Sigma}, \boldsymbol{D}) \quad \text { e } \quad \eta \sim H(\eta)
$$

em que $S \mathcal{N}_{J}(\boldsymbol{\mu}, \boldsymbol{\Sigma}, \boldsymbol{D})$ denota a distribuição normal assimétrica com vetor de parâmetros $(\boldsymbol{\mu}, \boldsymbol{\Sigma}, \boldsymbol{D})$ pertencente à classe de distribuições elípticas assimétricas apresentada na Definição 3. Consequentemente, temos que a distribuição $\boldsymbol{Z}$ condicionada a variável de mistura $\eta$ segue uma distribuição normal assimétrica dada por

$$
\boldsymbol{Z} \mid \eta \sim S_{\mathcal{N}}\left(\boldsymbol{\mu} ; \kappa(\eta) \boldsymbol{\Sigma}, \kappa(\eta)^{1 / 2} \boldsymbol{D}\right)
$$


Esta representação hierárquica nos permite escrever o modelo de regressão multivariado binário com função de ligação MENA da seguinte forma

$$
\begin{aligned}
P\left(\boldsymbol{Y}_{i}=\boldsymbol{y}_{i} \mid \boldsymbol{\beta}, \boldsymbol{\Gamma}, \boldsymbol{D}\right) & = \\
& \int_{B_{i J}} \ldots \int_{B_{i 1}} 2^{J}\left[\int_{0}^{\infty} \phi_{J}\left(\boldsymbol{t} \mid \boldsymbol{X}_{i} \boldsymbol{\beta}, \kappa(\eta)\left(\boldsymbol{\Gamma}+\boldsymbol{D}^{2}\right)\right) \times\right. \\
\Phi_{J} & \left.\left\{\boldsymbol{D}\left(\boldsymbol{\Gamma}+\boldsymbol{D}^{2}\right)^{-1}\left(\boldsymbol{t}-\boldsymbol{X}_{i} \boldsymbol{\beta}\right) \mid \mathbf{0},\left(\boldsymbol{I}-\boldsymbol{D}\left(\boldsymbol{\Gamma}+\boldsymbol{D}^{2}\right)^{-1} \boldsymbol{D}\right) \kappa(\eta)\right\} d H(\eta)\right] d \boldsymbol{t},
\end{aligned}
$$

em que $B_{i}=B_{i 1} \times B_{i 2} \times \ldots B_{i J}, \mathrm{e}$

$$
B_{i j}= \begin{cases}(0,+\infty) & \text { se } y_{i j}=1 \\ (-\infty, 0] & \text { se } y_{i j}=0\end{cases}
$$

Sahu, Dey e Branco (2003) utilizaram a seguinte abordagem para construir o modelo elíptico assimétrico apresentado na Definição 4.

Considere $\boldsymbol{\epsilon}$ e $\boldsymbol{W}$ dois vetores aleatórios $J$-dimensionais e seja $\boldsymbol{\Sigma}$ uma matriz positiva definida de dimensão $J \times J$ tal que

$$
\left(\begin{array}{c}
\boldsymbol{\epsilon} \\
\boldsymbol{W}
\end{array}\right) \sim E l_{2 J}\left(\left(\begin{array}{c}
\boldsymbol{\mu} \\
\mathbf{0}
\end{array}\right),\left(\begin{array}{cc}
\boldsymbol{\Sigma} & \mathbf{0} \\
\mathbf{0} & \boldsymbol{I}
\end{array}\right), g^{(2 J)}\right) .
$$

A distribuição resultante da transformação

$$
Z=\mu+D W+\epsilon
$$

tal que $[\boldsymbol{Z} \mid \boldsymbol{W}>\mathbf{0}]$ é a distribuição elíptica assimétrica apresentada na Definição 4 . O condicionamento $[\boldsymbol{Z} \mid \boldsymbol{W}>\mathbf{0}]$ significa dizer que cada $Z_{j}$ está condicionada nos valores de $W_{j}>0$ para $j=1, \ldots, J$.

É importante notar que esta construção nos permite representar a função de distribuição acumulada do vetor aleatório $\boldsymbol{Z}$ definido em (2.24) pode ser escrita em função da distribuição elíptica apresentada em (2.23) da seguinte forma

$$
P(\boldsymbol{Z} \leq \boldsymbol{z} \mid \boldsymbol{\mu}, \boldsymbol{D}, \boldsymbol{\Sigma})=F_{E L_{2 J}}\left(\left(\begin{array}{l}
\boldsymbol{z} \\
\mathbf{0}
\end{array}\right) ;\left(\begin{array}{c}
\boldsymbol{\mu} \\
\mathbf{0}
\end{array}\right),\left(\begin{array}{cc}
\boldsymbol{\Sigma}+\boldsymbol{D}^{2} & -\boldsymbol{D} \\
-\boldsymbol{D} & \boldsymbol{I}
\end{array}\right), g^{(2 J)}\right) .
$$

No caso particular em que a distribuição elíptica é normal, temos que a função de distribuição acumulada da distribuição multivariada normal assimétrica é dada por

$$
P(\boldsymbol{Z} \leq \boldsymbol{z} \mid \boldsymbol{\mu}, \boldsymbol{D}, \boldsymbol{\Sigma})=\Phi_{2 J}\left(\left(\begin{array}{l}
\boldsymbol{z} \\
\mathbf{0}
\end{array}\right) \mid\left(\begin{array}{c}
\boldsymbol{\mu} \\
\mathbf{0}
\end{array}\right),\left(\begin{array}{cc}
\boldsymbol{\Sigma}+\boldsymbol{D}^{2} & -\boldsymbol{D} \\
-\boldsymbol{D} & \boldsymbol{I}
\end{array}\right)\right),
$$

em que $\Phi_{2 J}(\boldsymbol{z} \mid \boldsymbol{\mu}, \boldsymbol{\Sigma})$ denota a função de distribuição acumulada de uma distribuição normal $2 J$-variada com vetor de parâmetros de localização $\boldsymbol{\mu}$ e matriz de escala $\boldsymbol{\Sigma}$ avaliada no ponto $\boldsymbol{z}$. 
A construção de distribuições elípticas multivariadas definida em (2.23)-(2.24) nos permite representar a distribuição multivariada normal assimétrica da seguinte forma

$$
Z_{0}=D W+\epsilon
$$

em que

$$
\boldsymbol{\epsilon} \sim \mathcal{N}_{J}(\mathbf{0}, \boldsymbol{\Sigma}) \quad \text { e } \quad \boldsymbol{W} \sim \mathcal{N}_{J}^{+}(\mathbf{0}, \boldsymbol{I})
$$

$\operatorname{com} \mathcal{N}_{J}^{+}(\boldsymbol{\mu}, \boldsymbol{\Sigma})$ representando a distrinuição normal $J$-variada positiva com vetor de parâmetros de localização $\boldsymbol{\mu} \in \mathbb{R}^{J}$ e matriz de escala $\boldsymbol{\Sigma} \in \mathbb{R}^{J \times J}$.

Combinando as representações estocásticas (2.21)-(2.22) e (2.27)-(2.28), temos a seguinte representação da distribuição de mistura no parâmetro de escala de distribuições normal assimétrica

$$
\boldsymbol{Z}=\boldsymbol{\mu}+\kappa(\eta)^{1 / 2}(\boldsymbol{D} \boldsymbol{W}+\boldsymbol{\epsilon})
$$

em que

$$
\begin{aligned}
\boldsymbol{\epsilon} & \sim \mathcal{N}_{J}(\mathbf{0}, \boldsymbol{\Sigma}) \\
\boldsymbol{W} & \sim \mathcal{N}_{J}^{+}(\mathbf{0}, \boldsymbol{I}) \\
\eta & \sim H .
\end{aligned}
$$

Logo, usando a abordagem de dados aumentados (Albert e Chib, 1993) para representar um modelo de regressão binária através de variáveis latentes, temos que o modelo regressão binário com função de ligação MENA pode ser equivalentemente representado como

$$
Y_{i j}= \begin{cases}1 & \text { se } z_{i j}>0 \\ 0 & \text { se } z_{i j} \leq 0\end{cases}
$$

em que

$$
\boldsymbol{Z}_{i}=\boldsymbol{X}_{i} \boldsymbol{\beta}+\kappa(\eta)^{1 / 2}\left(\boldsymbol{D} \boldsymbol{W}_{i}+\boldsymbol{\epsilon}_{i}\right)
$$

com

$$
\begin{aligned}
\boldsymbol{\epsilon}_{i} & \sim \mathcal{N}_{J}(\mathbf{0}, \boldsymbol{\Sigma}) \\
\boldsymbol{W}_{i} & \sim \mathcal{N}_{J}^{+}(\mathbf{0}, \boldsymbol{I}) \\
\eta_{i} & \sim H
\end{aligned}
$$

Na próxima seção nós apresentamos diversos exemplos de distribuições elípticas assimétricas que podem ser utilizadas como função de ligação para o modelo multivariado binário.

\subsubsection{Exemplos de distribuições assimétricas}

Mostramos, nesta seção, a formulação estocástica de alguns casos especiais de distribuições elípticas assimétricas pertencentes à classe de distribuições representáveis através de mistura de escala normal assimétrica. 


\section{Normal assimétrica}

A distribuição normal assimétrica (Sahu, Dey e Branco, 2003) é obtida quando a função de distribuição de mistura $H$ é degenerada no ponto $\eta=1$ e a função $\kappa(\eta)$ é igual a 1 para todo $\eta>0$. A função de densidade da distribuição multivariada normal assimétrica é

$$
\begin{aligned}
f(\boldsymbol{z} \mid \boldsymbol{\mu}, \boldsymbol{\Sigma}, \boldsymbol{D})= & 2^{J}\left|\boldsymbol{\Sigma}+\boldsymbol{D}^{2}\right|^{-\frac{1}{2}} \phi_{J}\left[\left(\boldsymbol{\Sigma}+\boldsymbol{D}^{2}\right)^{-\frac{1}{2}}(\boldsymbol{z}-\boldsymbol{\mu})\right] \times \\
& \Phi_{J}\left[\boldsymbol{D}\left(\boldsymbol{\Sigma}+\boldsymbol{D}^{2}\right)^{-1}(\boldsymbol{z}-\boldsymbol{\mu}) ; \mathbf{0}, I-\boldsymbol{D}\left(\boldsymbol{\Sigma}+\boldsymbol{D}^{2}\right)^{-1} \boldsymbol{D}\right],
\end{aligned}
$$

em que $\phi_{J}$ e $\Phi_{J}$ denotam, respectivamente, a função de densidade de probabilidade (fdp) e a função de distribuição acumulada (fda) da distribuição normal $J$-variada padrão. Denotamos esta distribuição por $\mathcal{S N}_{J}(\boldsymbol{\mu}, \boldsymbol{\Sigma}, \boldsymbol{D})$.

É importante notar que a distribuição normal assimétrica dada na expressão (2.34) se reduz a

$$
f(\boldsymbol{z} \mid \boldsymbol{\mu}, \boldsymbol{\Sigma}, \boldsymbol{D})=\prod_{j=1}^{J}\left[2\left(\sigma_{j}^{2}+\delta_{j}^{2}\right)^{-1} \phi\left(\frac{z_{j}-\mu_{j}}{\sqrt{\sigma_{j}^{2}+\delta_{j}^{2}}}\right) \Phi\left(\frac{\delta_{j}}{\sigma_{j}} \frac{z_{j}-\mu_{j}}{\sqrt{\sigma_{j}^{2}+\delta_{j}^{2}}}\right)\right],
$$

quando a matriz de escala é diagonal e dada por $\boldsymbol{\Sigma}=\operatorname{diag}\left(\sigma_{1}^{2}, \ldots, \sigma_{J}^{2}\right)$. Esta propriedade da distribuição normal assimétrica proposta por Sahu, Dey e Branco (2003) garante que distribuições marginais não correlacionadas implica em distribuições marginais independentes.

\section{Distribuição $t$ assimétrica}

A distribuição $t$ assimétrica é obtida quando $\kappa(\eta)=1 / \eta$ e a distribuição de mistura é uma distribuição gama. No caso particular em que $\eta \sim \operatorname{Gama}(\nu / 2, \nu / 2)$, cuja função de densidade de probabilidade é

$$
h(\eta \mid \nu / 2, \nu / 2)=\frac{(\nu / 2)^{\nu / 2}}{\Gamma(\nu / 2)} \eta_{i}^{\nu / 2-1} \exp \left(-\frac{\nu}{2} \eta_{i}\right),
$$

temos que a função de densidade da distribuição multivariada $t$ assimétrica é

$$
\begin{aligned}
& f(\boldsymbol{z} \mid \boldsymbol{\mu}, \boldsymbol{\Sigma}, \boldsymbol{D}, \nu)=2^{J} t_{J, \nu}\left(\boldsymbol{z} \mid \boldsymbol{\mu}, \boldsymbol{\Sigma}+\boldsymbol{D}^{2}\right) \times \\
& \quad T_{J, \nu+J}\left[\left(\frac{\nu+q(\boldsymbol{z}-\boldsymbol{\mu})}{\nu+J}\right)^{-\frac{1}{2}} \boldsymbol{D}\left(\boldsymbol{\Sigma}+\boldsymbol{D}^{2}\right)^{-1}(\boldsymbol{z}-\boldsymbol{\mu}) ; \mathbf{0}, \boldsymbol{I}+\boldsymbol{D}\left(\boldsymbol{\Sigma}+\boldsymbol{D}^{2}\right)^{-1} \boldsymbol{D}\right],
\end{aligned}
$$

em que $t_{J, \nu}$ e $T_{J, \nu}$ denotam, respectivamente, a fdp e a fda da distribuição $t$-Student $J$-variada com vetor de parâmetros de localização $\mathbf{0}$, matriz de escala identidade e $\nu$ graus de liberdade. Esta distribuição é denotada por $S T_{\nu}(\boldsymbol{\mu}, \boldsymbol{\Sigma}, \boldsymbol{D})$.

\section{Distribuição logística assimétrica}

A distribuição logística assimétrica é um caso particular do modelo MENA. Ela obtida quando $\kappa(\eta)=4 \eta^{2}$ e $\eta$ segue uma distribuição Kolmogorov assintótica, cuja função de densidade de proba- 
bilidade é dada por

$$
h(\eta)=8 \sum_{k=1}^{\infty}(-1)^{k+1} k^{2} \eta \exp \left\{-2 k^{2} \eta^{2}\right\}, \eta>0 .
$$

Esta forma de representar a distribuição logística foi introduzida por Choy (1995).

\section{Distribuição slash assimétrica}

A distribuição slash é obtida quando $\kappa(\eta)=\eta^{-1 / q}$ e $\eta \sim U(0,1)$, com $q>0$. Wang e Genton (2006) apresentam a versão assimétrica da distribuição slash multivariada, derivam diversas de suas propriedades e mostram que esta distribuição slash assimétrica é fácil de ser simulada. Arslan (2008) desenvolve uma alternativa multivariada para a distribuição slash assimétrica e a nomeia de distribuição slash assimétrica hiperbólica generalizada e mostra que esta distribuição é potencialmente útil para estudos de robustez.

\section{Distribuição exponencial dupla (Laplace) assimétrica}

A distribuição Laplace é um caso especial das distribuições MENA, obtida quando $\kappa(\eta)=1 /(4 \eta)$ e $\eta$ tem a seguinte função de densidade de probabilidade,

$$
h(\eta)=\frac{(1 / 4)^{1 / 2}}{\Gamma(1 / 2) \eta^{(1+J) / 2+1}} e^{-1 /(4 \eta)}, \eta>0
$$

\section{Distribuição stable assimétrica}

Distribuições stable univariadas são uma classe de distribuições limite para somas de variáveis aleatórias independentes e identicamente distribuídas. Elas são caracterizadas por quatro parâmetros, o índice de estabilidade $\alpha,[0<\alpha<2]$, o parâmetro de escala $\sigma,[\sigma>0]$, o parâmetro de assimetria $\gamma,[-1<\gamma<1]$ e o parâmetro mudança $\mu,[\infty<\mu<\infty]$. O parâmetro $\alpha$ quantifica o grau de pico e de peso das caldas da distribuição stable. Ajustando o parâmetro de assimetria em $\gamma=0$, temos uma distribuição stable simétrica, fazendo também $\alpha=0$, temos que a distribuição stable corresponde a distribuição normal. A variância da distribuição stable torna-se infinita quando $\alpha<2$. Esta característica faz a distribuição stable útil para ajustar dados que admitem valores extremos.

A distribuição stable multivariada assimétrica é obtida quando $\kappa(\eta)=2 \eta \mathrm{e}$

$$
h(\eta)=S^{P}(\eta \mid \alpha, 1)=\frac{\alpha}{1-\alpha} \eta^{-\left(\frac{\alpha}{1-\alpha}+1\right)} \int_{0}^{1} s(u) \exp \left\{-\frac{s(u)}{\eta^{\frac{\alpha}{1-\alpha}}}\right\} d u, 0<\alpha<1,
$$

com

$$
s(u)=\left(\frac{\sin (\alpha \pi u)}{\sin (\pi u)}\right)^{\frac{\alpha}{1-\alpha}}\left(\frac{\sin [(1-\alpha) \pi u]}{\sin (\pi u)}\right) .
$$

A distribuição stable multivariada simétrica $S_{J}\left(2 \alpha, \mathbf{0}, \boldsymbol{X}_{i} \boldsymbol{\beta}, \boldsymbol{\Sigma}\right)$, é caracterizada pelo logaritmo 
de sua função característica, a qual é dada por

$$
\ln \psi(t)=i\left(\boldsymbol{X}_{i} \boldsymbol{\beta}\right)^{\prime} t-\left(t^{\prime} \boldsymbol{\Sigma} t\right)^{\alpha}, \text { para } \alpha \in[1 / 2,1)
$$

com $t=\left(t_{1}, \ldots, t_{J}\right)^{\prime}$ e $i^{2}=-1$. Note que quando $\alpha=1 / 2$, temos que $S_{J}\left(1, \mathbf{0}, \boldsymbol{X}_{i} \boldsymbol{\beta}, \boldsymbol{\Sigma}\right)$ é uma distribuição Cauchy multivariada, enquanto que, quando $\alpha \rightarrow 1$, temos que $S_{J}\left(2, \mathbf{0}, \boldsymbol{X}_{i} \boldsymbol{\beta}, \boldsymbol{\Sigma}\right)=$ $\lim _{\alpha \rightarrow 1} S_{J}\left(2 \alpha, \mathbf{0}, \boldsymbol{X}_{i} \boldsymbol{\beta}, \boldsymbol{\Sigma}\right)$ é uma distribuição normal multivariada. Portanto, a distribuição multivariada Cauchy assimétrica é um caso especial da distribuição multivariada $t$ assimétrica e da distribuição multivariada stable assimétrica. Além disso, a distribuição multivariada normal assimétrica é um caso limite da distribuição multivariada stable assimétrica quando $\alpha=0$.

\section{Distribuição exponencial potência}

A família de distribuições multivariadas exponencial potência assimétricas podem ser utilizadas quando o objetivo é obter funções de ligação robustas. A função de densidade de probabilidade da distribuição exponencial potência multivariada é dada por

$$
\pi_{E P}(\boldsymbol{z} \mid \boldsymbol{\mu}, \boldsymbol{\Sigma}, \alpha)=c_{J}|\boldsymbol{\Sigma}|^{-1 / 2} \exp \left\{-\left[c_{0}(\boldsymbol{z}-\boldsymbol{\mu})^{\prime} \boldsymbol{\Sigma}^{-1}(\boldsymbol{z}-\boldsymbol{\mu})\right]^{\alpha}\right\}, 1 / 2 \leq \alpha \leq 1,
$$

em que $\alpha$ denota o parâmetro de curtose e as constantes $c_{0}$ e $c_{J}$ são definidas como

$$
c_{0}=\frac{\Gamma\left(\frac{3}{2 \alpha}\right)}{\Gamma\left(\frac{1}{2 \alpha}\right)} \text { e } \quad c_{J}=\frac{\alpha c_{0}^{J / 2} \Gamma\left(\frac{J}{2}\right)}{\Gamma\left(\frac{J}{2 \alpha}\right) \pi^{J / 2}} .
$$

Segue de Andrews e Mallows (1974), West (1987), e Choy (1995), que a distribuição multivariada exponencial potência é também um caso especial da família de mistura de escala normal, e consequentemente, a distribuição exponencial potência assimétrica é um caso especial das ditribuições MENA quando $\kappa(\eta)=1 /\left(2 c_{0} \eta\right)$ e $h(\eta)=\left(\frac{1}{\eta}\right)^{J / 2} S^{P}(\eta \mid \alpha, 1)$, em que $S^{P}(\eta \mid \alpha, 1)$ é definido em (2.38). A distribuição normal assimétrica $(\alpha=1)$ e a distribuição Laplace assimétrica $(\alpha=1 / 2)$ são casos particulares da distribuição exponencial potência.

A Tabela 2.2 lista várias distribuições que podem ser obtidas através de mistura no parâmetro de escala da distribuição normal.

Tabela 2.2: Algumas funções de mistura e suas respectivas variáveis misturadoras

\begin{tabular}{llll}
\hline Distribuição & Notação & Função de mistura & variável da mistura \\
\hline Normal & $\mathcal{N}_{J}(\boldsymbol{\mu}, \kappa(\eta) \boldsymbol{\Sigma})$ & $\kappa(\eta)=1$ & - \\
t-Student & $S T_{J}(\boldsymbol{\mu}, \kappa(\eta) \boldsymbol{\Sigma}, \nu)$ & $\kappa(\eta)=1 / \eta$ & $\eta \sim \operatorname{Gama}(\nu / 2, \nu / 2)$ \\
Logística & $L_{J}(\boldsymbol{\mu}, \kappa(\eta) \boldsymbol{\Sigma})$ & $\kappa(\eta)=4 \eta^{2}$ & $\eta \sim K S$ \\
Slash & $S l_{J}(\boldsymbol{\mu}, \kappa(\eta) \boldsymbol{\Sigma})$ & $\kappa(\eta)=\eta^{-1 / q}$ & $\eta \sim U(0,1)$ \\
Stable & $S t_{J}(\boldsymbol{\mu}, \kappa(\eta) \boldsymbol{\Sigma})$ & $\kappa(\eta)=2 \eta$ & $\eta \sim S^{P}(\eta \mid \alpha, 1)$ \\
Exponencial duplo & $E D_{J}(\boldsymbol{\mu}, \kappa(\eta) \boldsymbol{\Sigma})$ & $\kappa(\eta)=1 /(4 \eta)$ & $\eta \sim I G(1 / 4,1 / 2)$ \\
Exponencial potência & $E P_{J}(\boldsymbol{\mu}, \kappa(\eta) \boldsymbol{\Sigma}, \lambda)$ & $\kappa(\eta)=1 /\left(2 c_{0} \eta\right)$ & $\eta \sim\left(\frac{1}{\eta}\right)^{J / 2} S^{P}(\eta \mid \alpha, 1)$ \\
\hline
\end{tabular}




\section{Capítulo 3}

\section{Inferência em Modelos Assimétricos}

Considere $\boldsymbol{Y}_{1}, \boldsymbol{Y}_{2}, \ldots, \boldsymbol{Y}_{n}$ uma amostra aleatória de $n$ vetores aleatórios independentes de dimensão $J$. Considere também que cada elemento $\boldsymbol{Y}_{i}=\left(Y_{i 1}, Y_{i 2}, \ldots, Y_{i J}\right)^{\prime}$ desta amostra está associado a uma matriz $\boldsymbol{X}_{i}$ de variáveis explicativas para todo $i=1, \ldots, n$, e que cada componente deste vetor de respostas é uma variável aleatória $Y_{i j}, j=1, \ldots, J$, que toma somente dois valores, denotados aqui por 0 e 1 . Do capítulo 2, temos que o modelo multivariado de mistura de escala normal assimétrica (MENA) é uma alternativa para o ajuste destes tipos de conjuntos de dados com respostas multivariadas binárias. A função de verossimilhança do MENA é dada por

$$
L\left(\boldsymbol{\beta}, \boldsymbol{\Gamma}, \boldsymbol{D} ; \boldsymbol{y}_{1}, \boldsymbol{y}_{2}, \ldots, \boldsymbol{y}_{n}\right)=\prod_{i=1}^{n} P\left(\boldsymbol{Y}_{i}=\boldsymbol{y}_{i} \mid \boldsymbol{\beta}, \boldsymbol{\Gamma}, \boldsymbol{D}\right),
$$

em que

$$
\begin{aligned}
& P\left(\boldsymbol{Y}_{i}=\boldsymbol{y}_{i} \mid \boldsymbol{\beta}, \boldsymbol{\Gamma}, \boldsymbol{D}\right)= \\
& \int_{A_{i J}} \ldots \int_{A_{i 1}} 2^{J}\left[\int_{0}^{\infty} \phi_{J}\left(\boldsymbol{t} \mid \mathbf{0}, \kappa(\eta)\left(\boldsymbol{\Gamma}+\boldsymbol{D}^{2}\right)\right) \times\right. \\
&\left.\Phi_{J}\left\{\left[\left(\boldsymbol{I}-\boldsymbol{D}\left(\boldsymbol{\Gamma}+\boldsymbol{D}^{2}\right)^{-1} \boldsymbol{D}\right) \kappa(\eta)\right]^{-1 / 2} \boldsymbol{D}\left(\boldsymbol{\Gamma}+\boldsymbol{D}^{2}\right)^{-1} \boldsymbol{t} \mid \mathbf{0}, \boldsymbol{I}\right\} d H(\eta)\right] d \boldsymbol{t},
\end{aligned}
$$

denota a probabilidade do vetor aleatório $\boldsymbol{Y}_{i}$ ser igual ao vetor observado $\boldsymbol{y}_{i}$. O vetor de parâmetros deste modelo é denotado por $\boldsymbol{\theta}=(\boldsymbol{\beta}, \boldsymbol{\Gamma}, \boldsymbol{D})$, em $\boldsymbol{\beta}=\left(\boldsymbol{\beta}_{1}, \boldsymbol{\beta}_{2}, \ldots, \boldsymbol{\beta}_{J}\right)$ é o vetor de coeficientes regressores, $\boldsymbol{\Gamma}$ a matriz de escala e $\boldsymbol{D}=\operatorname{diag}(\boldsymbol{\delta})$ é uma matriz diagnonal de forma, com $\boldsymbol{\delta}=$ $\left(\delta_{1}, \ldots, \delta_{J}\right)$. A função $\kappa(\eta)>0$ é uma função positiva associada a variável de mistura $\eta$, em que $\eta$ é uma variável estritamente positiva com função de distribuição acumulada $H$, a qual pode depender de parâmetros adicionais, por exemplo, os graus de liberdade $\nu$ nos modelos $t$-Student. Os intervalos de integração $A_{i j}(i=1, \ldots n, j=1, \ldots J)$, são dados por

$$
A_{i j}= \begin{cases}\left(-\infty, \boldsymbol{x}_{i j}^{\prime} \boldsymbol{\beta}_{j}\right] & \text { se } y_{i j}=1 \\ \left(\boldsymbol{x}_{i j}^{\prime} \boldsymbol{\beta}_{j},+\infty\right) & \text { se } y_{i j}=0\end{cases}
$$

O modelo multivariado de mistura de escala normal assimétrica é indexado por três grupos distintos de parâmetros. Um grupo de parâmetros de localização $\boldsymbol{\beta}$, um de escala $\boldsymbol{\Gamma}$ e um de forma $\boldsymbol{\delta}$. Desde que não há estudos na literatura sobre uma possível relação de dependência entre estes grupos, torna-se extremamente importante e necessário a verificação da identificabilidade do 
modelo antes de realizar inferências. Algumas restrições no espaço paramétrico foram considerados por Chib e Greenberg (1998) no modelo multivariado probito e por Chen e Dey (1998) nos modelos multivariados de mistura de escala normal (MEN). No entanto, estes autores não apresentam estudos sobre a falta identificabilidade em seus respectivos modelos propostos. A próxima seção apresenta um estudo sobre identificabilidade para o modelo MENA e, como os modelos multivariados probito e de mistura de escala normal são casos particulares dos modelos MENA, a próxima seção preenche a lacuna na literatura para os modelos multivariados probito (Chib e Greenberg, 1998) e de mistura de escala normal (Chen e Dey, 1998).

\subsection{Identificabilidade do modelo}

Um modelo de regressão é considerado não identificável quando o vetor de parâmetros desconhecidos $\boldsymbol{\theta}$ é não identificável para a família de modelos indexados pelo vetor de parâmetros $\boldsymbol{\theta}$. Considere a seguinte definição de parâmetro não identificável para uma família de distribuições de probabilidade.

Definição 5. Seja $U$ uma variável aleatória observável com função de distribuição $F_{\boldsymbol{\theta}}$, com $F_{\boldsymbol{\theta}}$ pertencente à família $\mathcal{F}=\left\{\mathcal{F}_{\boldsymbol{\theta}}: \boldsymbol{\theta} \in \boldsymbol{\Theta}\right\}$ de distribuiçôes indexadas pelo parâmetro $\boldsymbol{\theta}$. Aqui, $\boldsymbol{\theta}$ pode ser um escalar ou um vetor. Nós dizemos que $\boldsymbol{\theta}$ é não identificável por $U$ se existe pelo menos um par $\left(\boldsymbol{\theta}, \boldsymbol{\theta}^{*}\right)$, em que $\boldsymbol{\theta}$ e $\boldsymbol{\theta}^{*}$ pertencem à $\boldsymbol{\Theta}$ tal que $F_{\boldsymbol{\theta}}(u)=F_{\boldsymbol{\theta}^{*}}(u)$ para todo u. Caso contrário diremos que $\boldsymbol{\theta}$ é identificável.

Considere, sem perda de generalidade, que $\boldsymbol{Y}=\left(Y_{1}, Y_{2}, \ldots, Y_{J}\right)$ é uma amostra de tamanho um do vetor aleatório definido em (3.1). A verossimilhança do modelo multivariado de mistura de escala normal assimétrica (MENA) com vetor de parâmetros $(\boldsymbol{\beta}, \boldsymbol{\Gamma}, \boldsymbol{D})$ baseado em uma única observação é definida como

$$
L(\boldsymbol{\beta}, \boldsymbol{\Gamma}, \boldsymbol{D} ; \boldsymbol{y})=P(\boldsymbol{Y}=\boldsymbol{y} \mid \boldsymbol{\beta}, \boldsymbol{\Gamma}, \boldsymbol{D})=\int_{0}^{\infty} P(\boldsymbol{Y}=\boldsymbol{y} \mid \boldsymbol{\beta}, \kappa \boldsymbol{\Gamma}, \sqrt{\kappa} \boldsymbol{D}) d H(\eta),
$$

em que

$$
\begin{aligned}
& P(\boldsymbol{Y}=\boldsymbol{y} \mid \boldsymbol{\beta}, \kappa \boldsymbol{\Gamma}, \sqrt{\kappa} \boldsymbol{D})= \\
& \quad \int_{A_{J}} \ldots \int_{A_{1}} 2^{J} \phi_{J}\left(\boldsymbol{t} \mid \mathbf{0}, \kappa\left(\boldsymbol{\Gamma}+\boldsymbol{D}^{2}\right)\right) \Phi_{J}\left\{\boldsymbol{D}\left(\boldsymbol{\Gamma}+\boldsymbol{D}^{2}\right)^{-1} \boldsymbol{t} \mid \mathbf{0},\left(\boldsymbol{I}-\boldsymbol{D}\left(\boldsymbol{\Gamma}+\boldsymbol{D}^{2}\right)^{-1} \boldsymbol{D}\right) \kappa\right\} d \boldsymbol{t},
\end{aligned}
$$

$\kappa=\kappa(\eta)$ é uma função de mistura e os intervalos $A_{j}, j=1, \ldots, J$, são definidos por

$$
A_{j}= \begin{cases}\left(-\infty, \boldsymbol{x}_{j} \boldsymbol{\beta}_{j}\right] & \text { se } y_{j}=1 \\ \left(\boldsymbol{x}_{j} \boldsymbol{\beta}_{j},+\infty\right) & \text { se } y_{j}=0\end{cases}
$$

Note que a probabilidade $P(\boldsymbol{Y}=\boldsymbol{y} \mid \boldsymbol{\beta}, \kappa \boldsymbol{\Gamma}, \sqrt{\kappa} \boldsymbol{D})$ dada em (3.4) é o modelo multivariado probito assimétrico com vetor de parâmetros $(\boldsymbol{\beta}, \kappa \boldsymbol{\Gamma}, \sqrt{\kappa} \boldsymbol{D})$. Também é importante observar que a matriz $\boldsymbol{\Gamma}+\boldsymbol{D}^{2}$ é definida positiva e, portanto, pode ser escrita da seguinte forma

$$
\boldsymbol{\Gamma}+\boldsymbol{D}^{2}=\boldsymbol{S} \boldsymbol{\Sigma} \boldsymbol{S}
$$


em que $\boldsymbol{S}=\operatorname{diag}\left(s_{1}, s_{2}, \ldots, s_{J}\right)$ é a seguinte matriz diagonal

$$
\boldsymbol{S}=\left(\begin{array}{ccccc}
\sqrt{\gamma_{11}+\delta_{1}^{2}} & 0 & 0 & \cdots & 0 \\
0 & \sqrt{\gamma_{22}+\delta_{2}^{2}} & 0 & \cdots & 0 \\
\vdots & \cdots & \ddots & \cdots & \vdots \\
0 & \cdots & 0 & \sqrt{\gamma_{J-1, J-1}+\delta_{J-1}^{2}} & 0 \\
0 & \cdots & 0 & 0 & \sqrt{\gamma_{J J}+\delta_{J}^{2}}
\end{array}\right)
$$

e $\boldsymbol{\Sigma}$ é uma matriz definida positiva dada por

$$
\boldsymbol{\Sigma}=\left(\begin{array}{ccccc}
1 & \frac{\gamma_{12}}{s_{1} s_{2}} & \frac{\gamma_{13}}{s_{1} s_{3}} & \cdots & \frac{\gamma_{1 J}}{s_{1} s_{J}} \\
\frac{\gamma_{21}}{s_{2} s_{1}} & 1 & \frac{\gamma_{23}}{s_{2} s_{3}} & \cdots & \frac{\gamma_{2}}{s_{2} s_{J}} \\
\vdots & \cdots & \ddots & \ldots & \vdots \\
\frac{\gamma_{J-1,1}}{s_{J-1} s_{1}} & \cdots & \frac{\gamma_{J-1, J-2}}{s_{J-1} s_{J-2}} & 1 & \frac{\gamma_{J-1, J}}{\gamma_{J-1} s_{J}} \\
\frac{\gamma_{J 1}}{s_{J} s_{1}} & \cdots & \frac{\gamma_{J, J}}{s_{J} s_{J-2}} & \frac{\gamma_{J, J-1}}{s_{J} s_{J-1}} & 1
\end{array}\right)
$$

Isso implica que o modelo multivariado probito assimétrico apresentado em (3.4) pode ser representado através das matrizes $\boldsymbol{\Sigma}$ e $\boldsymbol{S}$ como

$$
\begin{aligned}
& P(\boldsymbol{Y}=\boldsymbol{y} \mid \boldsymbol{\beta}, \kappa \boldsymbol{\Gamma}, \sqrt{\kappa} \boldsymbol{D})= \\
& \quad \int_{A_{J}} \ldots \int_{A_{1}} 2^{J} \phi_{J}(\boldsymbol{t} \mid \mathbf{0}, \kappa \boldsymbol{S} \boldsymbol{\Sigma} \boldsymbol{S}) \Phi_{J}\left\{\boldsymbol{D}(\boldsymbol{S} \boldsymbol{\Sigma} \boldsymbol{S})^{-1} \boldsymbol{t} \mid \mathbf{0},\left(\boldsymbol{I}-\boldsymbol{D}(\boldsymbol{S} \boldsymbol{\Sigma} \boldsymbol{S})^{-1} \boldsymbol{D}\right) \kappa\right\} d \boldsymbol{t} .
\end{aligned}
$$

Esta transformação paramétrica torna mais clara a visualização da relação de dependência entre os parâmetros de interesse. Considere agora a seguinte transformação de variáveis,

$$
\boldsymbol{u}=\boldsymbol{S}^{-1} \boldsymbol{t}=\left(\frac{t_{1}}{\sqrt{\gamma_{11}+\delta_{1}^{2}}}, \frac{t_{2}}{\sqrt{\gamma_{22}+\delta_{2}^{2}}}, \ldots, \frac{t_{J}}{\sqrt{\gamma_{J J}+\delta_{J}^{2}}}\right)^{\prime}
$$

em que $\boldsymbol{S}$ é matriz diagonal dada em (3.7). É fácil notar que determinante do jacobiano desta transformação é dado por

$$
|J: \boldsymbol{t} \rightarrow \boldsymbol{u}|=\left|\frac{d \boldsymbol{t}}{d \boldsymbol{u}}\right|=|\boldsymbol{S}|=\prod_{j=1}^{J} \sqrt{\gamma_{j j}+\delta_{j}^{2}}
$$

e o resultado da transformação de variáveis apresentada em (3.8) é a seguinte integral

$$
\begin{aligned}
& P(\boldsymbol{Y}=\boldsymbol{y} \mid \boldsymbol{\beta}, \kappa \boldsymbol{\Gamma}, \sqrt{\kappa} \boldsymbol{D})= \\
& \int_{A_{J}^{*}} \ldots \int_{A_{1}^{*}} 2^{J} \phi_{J}(\boldsymbol{S u} \mid \mathbf{0}, \kappa \boldsymbol{S} \boldsymbol{\Sigma} \boldsymbol{S}) \Phi_{J}\left\{\left[\left(\boldsymbol{I}-\boldsymbol{D}(\boldsymbol{S} \boldsymbol{\Sigma} \boldsymbol{S})^{-1} \boldsymbol{D}\right) \kappa\right]^{-1 / 2} \boldsymbol{D}(\boldsymbol{S} \boldsymbol{\Sigma} \boldsymbol{S})^{-1} \boldsymbol{S} \boldsymbol{u} \mid \mathbf{0}, \boldsymbol{I}\right\}|\boldsymbol{S}| d \boldsymbol{u}= \\
& \int_{A_{J}^{*}} \ldots \int_{A_{1}^{*}} 2^{J} \phi_{J}(\boldsymbol{u} \mid \mathbf{0}, \kappa \boldsymbol{\Sigma}) \Phi_{J}\left\{\left[\left(\boldsymbol{I}-\left(\boldsymbol{D} \boldsymbol{S}^{-1}\right) \boldsymbol{\Sigma}^{-1}(\boldsymbol{D} \boldsymbol{S})^{-1}\right)^{\prime} \kappa\right]^{-1 / 2}\left(\boldsymbol{D} \boldsymbol{S}^{-1}\right) \boldsymbol{\Sigma}^{-1} \boldsymbol{u} \mid \mathbf{0}, \boldsymbol{I}\right\} d \boldsymbol{u}
\end{aligned}
$$


com

$$
A_{j}^{*}=\left\{\begin{array}{l}
\left(-\infty, \boldsymbol{x}_{j} \frac{1}{\sqrt{\gamma_{j j}+\delta_{j}^{2}}} \boldsymbol{\beta}_{j}\right] \\
\left(\boldsymbol{x}_{j} \frac{1}{\sqrt{\gamma_{j j}+\delta_{j}^{2}}} \boldsymbol{\beta}_{j},+\infty\right) \text { se } y_{j}=1, \\
\text { se } y_{j}=0 .
\end{array}\right.
$$

Temos também, da transformação paramétrica definida em (3.8), que

$$
\boldsymbol{\Sigma}=\boldsymbol{S}^{-1} \boldsymbol{\Gamma} \boldsymbol{S}^{-1}+\boldsymbol{S}^{-1} \boldsymbol{D}^{2} \boldsymbol{S}^{-1}=\boldsymbol{\Gamma}_{*}+\boldsymbol{D}_{*}^{2} .
$$

Logo, fazendo

$$
\boldsymbol{\Gamma}_{*}=\boldsymbol{S}^{-1} \boldsymbol{\Gamma} \boldsymbol{S}^{-1}, \boldsymbol{D}_{*}=\boldsymbol{D} \boldsymbol{S}^{-1} \text { e } \boldsymbol{\beta}_{j}^{*}=\frac{\boldsymbol{\beta}_{j}}{s_{j}}, \text { para } j=1, \ldots, J
$$

temos que o modelo multivariado probito assimétrico dado em (3.4) pode ser escrito como

$$
\begin{aligned}
& P(\boldsymbol{Y}=\boldsymbol{y} \mid \boldsymbol{\beta}, \kappa \boldsymbol{\Gamma}, \sqrt{\kappa} \boldsymbol{D})= \\
& \quad \int_{A_{J}^{*}} \ldots \int_{A_{1}^{*}} 2^{J} \phi_{J}\left(\boldsymbol{t} \mid \mathbf{0}, \kappa\left(\boldsymbol{\Gamma}_{*}+\boldsymbol{D}_{*}^{2}\right)\right) \Phi_{J}\left\{\left[\left(\boldsymbol{I}-\boldsymbol{D}_{*}\left(\boldsymbol{\Gamma}_{*}+\boldsymbol{D}_{*}^{2}\right)^{-1} \boldsymbol{D}_{*}\right) \kappa\right]^{-1 / 2} \boldsymbol{D}_{*}\left(\boldsymbol{\Gamma}_{*}+\boldsymbol{D}_{*}^{2}\right)^{-1} \boldsymbol{t} \mid \mathbf{0}, \boldsymbol{I}\right\} d \boldsymbol{t},
\end{aligned}
$$

em que

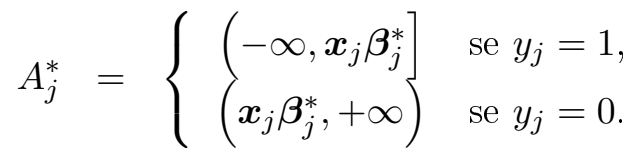

Portanto, temos a seguinte equivalência entre os modelos probito assimétricos.

$$
P(\boldsymbol{Y}=\boldsymbol{y} \mid \boldsymbol{\beta}, \kappa \boldsymbol{\Gamma}, \sqrt{\kappa} \boldsymbol{D})=P\left(\boldsymbol{Y}=\boldsymbol{y} \mid \boldsymbol{\beta}^{*}, \kappa \boldsymbol{\Gamma}_{*}, \sqrt{\kappa} \boldsymbol{D}_{*}\right),
$$

Além disto, considerando que a distribuição de $H(\eta)$ não depende do vetor de parâmetros $(\boldsymbol{\beta}, \boldsymbol{\Gamma}, \boldsymbol{D})$, temos, da expressão (3.4), que

$$
\begin{aligned}
P(\boldsymbol{Y}=\boldsymbol{y} \mid \boldsymbol{\beta}, \boldsymbol{\Gamma}, \boldsymbol{D}) & =\int_{0}^{\infty} P(\boldsymbol{Y}=\boldsymbol{y} \mid \boldsymbol{\beta}, \kappa \boldsymbol{\Gamma}, \sqrt{\kappa} \boldsymbol{D}) d H(\eta)= \\
& \int_{0}^{\infty} P\left(\boldsymbol{Y}=\boldsymbol{y} \mid \boldsymbol{\beta}^{*}, \kappa \boldsymbol{\Gamma}_{*}, \sqrt{\kappa} \boldsymbol{D}_{*}\right) d H(\eta)=P\left(\boldsymbol{Y}=\boldsymbol{y} \mid \boldsymbol{\beta}^{*}, \boldsymbol{\Gamma}_{*}, \boldsymbol{D}_{*}\right)
\end{aligned}
$$

Logo, as funções de verossimilhanças dos modelos multivariados MENA com vetores de parâmetros $\boldsymbol{\theta}=(\boldsymbol{\beta}, \boldsymbol{\Gamma}, \boldsymbol{D})$ e $\boldsymbol{\theta}^{*}=\left(\boldsymbol{\beta}^{*}, \boldsymbol{\Gamma}_{*}, \boldsymbol{D}_{*}\right)$ são equivalentes. Isto é

$$
L(\boldsymbol{\beta}, \boldsymbol{\Gamma}, \boldsymbol{D} ; \boldsymbol{y})=L\left(\boldsymbol{\beta}^{*}, \boldsymbol{\Gamma}_{*}, \boldsymbol{D}_{*} ; \boldsymbol{y}\right)
$$

Este resultado mostra que o modelo MENA com vetor de parâmetros $(\boldsymbol{\beta}, \boldsymbol{\Gamma}, \boldsymbol{D})$ é não identificável quando assume-se que $\Gamma$ é uma matriz de escala definida positiva irrestrita e $\boldsymbol{D}$ é uma matriz diagonal com seus componentes com variação na reta real. Portanto, algumas restrições no espaço 
paramétrico devem ser impostas para tornar o modelo identificável.

\subsubsection{Casos particulares de falta de identificabilidade}

Uma restrição para o modelo MENA é considerar que os parâmetros de formas são todos nulos, ou seja, assumir o caso simétrico. Sob esta restrição temos o seguinte modelo multivariado

$$
P(\boldsymbol{Y}=\boldsymbol{y} \mid \boldsymbol{\beta}, \boldsymbol{\Gamma})=\int_{A_{J}} \ldots \int_{A_{1}}\left(\int_{0}^{\infty} \frac{1}{(2 \pi)^{J / 2}|\kappa(\eta) \boldsymbol{\Gamma}|^{1 / 2}} \exp \left\{-\frac{1}{2 \kappa(\eta)} \boldsymbol{u}^{\prime} \boldsymbol{\Gamma}^{-1} \boldsymbol{u}\right\} d H(\eta)\right) d \boldsymbol{u} .
$$

com

$$
A_{j}= \begin{cases}\left(-\infty, \boldsymbol{x}_{j}^{\prime} \boldsymbol{\beta}_{j}\right. & \text { se } y_{j}=1 \\ \left(\boldsymbol{x}_{j}^{\prime} \boldsymbol{\beta}_{j},+\infty\right) & \text { se } y_{j}=0\end{cases}
$$

Pela seção anterior, pode-se mostrar que o modelo multivariado simétrico obtido via mistura de escala normal (MEN) com vetor de parâmetros $(\boldsymbol{\beta}, \boldsymbol{\Gamma})$ apresentado em (3.9) é equivalente ao modelo MEN com vetor de parâmetros $\left(\boldsymbol{\beta}^{*}, \boldsymbol{\Gamma}_{*}\right)$, em que

$$
\boldsymbol{\Gamma}_{*}=\boldsymbol{S}^{-1} \boldsymbol{\Gamma} \boldsymbol{S}^{-1} \text { e } \boldsymbol{\beta}_{j}^{*}=\frac{\boldsymbol{\beta}_{j}}{\sqrt{\gamma_{j j}}}, j=1, \ldots, J
$$

com

$$
\boldsymbol{S}=\operatorname{diag}\left(\sqrt{\gamma_{11}}, \sqrt{\gamma_{22}}, \ldots, \sqrt{\gamma_{J J}}\right) .
$$

Logo, o modelo multivariado com função de ligação de mistura de escala normal, e consequentemente, seus casos particulares, por exemplo, os modelos multivariados probito e logístico, são não identificáveis quando assume-se que a matriz de dispersão $\Gamma$ é uma matriz de covariâncias não estruturada. McCulloch e Rossi (1994) verificaram que este problema de falta de identificabilidade também ocorre no modelo multinomial probito. No entanto, os autores ignoraram a falta de identificabilidade e trabalharam com o modelo não identificável. Mais recentemente, McCulloch et al. (2000) resolveram este problema de não identificabilidade no modelo multinomial fazendo com que o primeiro elemento da diagonal da matriz de dispersão $\boldsymbol{\Gamma}$ seja igual a $1\left(\gamma_{11}=1\right)$ e Nobile (2000) sugeriu normalizar a matriz $\boldsymbol{\Gamma}$. No modelo multivariado probito, Chib e Greenberg (1998) resolvem a não identificabilidade assumindo que $\boldsymbol{\Gamma}$ seja uma matriz de correlações. Chen e Dey (1998) também consideram a mesma restrição sugerida por Chib e Greenberg (1998) na estimação dos parâmetros dos modelos multivariados de mistura de escala normal. Nós também consideraremos esta restrição na estimação de modelos multivariados simétricos. Ou seja, consideramos que $\gamma_{j j}=1$ para todo $j=1, \ldots, J$ quando $\delta_{j}=0$ para todo $j=1, \ldots, J$.

Diferentemente do caso simétrico, fixar $\gamma_{j j}$ igual a 1 (um) para todo $j=1, \ldots, J$ não torna o modelo identificável quando o espaço paramétrico do vetor de parâmetros de forma $\boldsymbol{\delta}$ é o espaço $\mathbb{R}^{J}$. Neste caso, temos que as funções de verossimilhanças dos modelos MENA com vetores de parâmetros $\boldsymbol{\theta}=(\boldsymbol{\beta}, \boldsymbol{\Gamma}, \boldsymbol{D})$ e $\boldsymbol{\theta}^{*}=\left(\boldsymbol{\beta}^{*}, \boldsymbol{\Gamma}_{*}, \boldsymbol{D}_{*}\right)$ apresentado em (3.1) são equivalentes quando

$$
\boldsymbol{\Gamma}_{*}=\boldsymbol{S}^{-1} \boldsymbol{\Gamma} \boldsymbol{S}^{-1}, \boldsymbol{D}_{*}=\boldsymbol{D} \boldsymbol{S}^{-1} \text { e } \boldsymbol{\beta}_{j}^{*}=\frac{\boldsymbol{\beta}_{j}}{\sqrt{1+\delta_{j}^{2}}}, j=1, \ldots, J
$$


em que

$$
\boldsymbol{S}=\operatorname{diag}\left(\sqrt{1+\delta_{1}^{2}}, \sqrt{1+\delta_{2}^{2}}, \ldots, \sqrt{1+\delta_{J}^{2}}\right)
$$

e $\boldsymbol{\Gamma}$ é uma matriz de correlações. Logo, o modelo é não identificável ao assumir somente que a matriz de escala $\boldsymbol{\Gamma}$ é uma matriz de correlações. Outras restrições no espaço paramétrico devem ser feitas para garantir a identificabilidade do modelo. Uma alternativa é assumir adicionalmente que o vetor de parâmetros de forma $\boldsymbol{\delta}$ é fixo e conhecido. Nesta abordagem, as estimativas dos parâmetros de forma devem ser obtidos através de uma grade de valores plausíveis para $\boldsymbol{\delta}$. Estes valores podem ser escolhidos de tal forma que uma função de perda pré-definida seja minimizada. Outra forma de tornar o modelo identificável é considerar que a soma $\gamma_{j j}+\delta_{j}^{2}$ é fixa e conhecida para todo $j=1, \ldots, J$. Embora o conceito de identificabilidade do modelo aqui utilizado, seja um conceito frequentista, este tipo de falta de identificabilidade pode afetar o procedimento bayesiano. Conforme discutido em Carlin e Louis (1996), Johnson et al. (2001), Rannala (2002), Swartz et al. (2004) e Shariati et al. (2009).

A próxima seção apresenta alguns algoritmos de Monte Carlo via Cadeias de Markov (MCMC) que podem ser utilizados para estimar os parâmetros de interesse do modelo MENA através de inferência bayesiana.

\subsection{Algoritmos MCMC}

A função verossimilhança do modelo multivariado de mistura de escala normal assimétrica dado em (3.1) pode ser reescrito de modo que os limites de integração não sejam funções de parâmetros de interesse da seguinte forma

$$
L\left(\boldsymbol{\beta}, \boldsymbol{\Gamma}, \boldsymbol{D} ; \boldsymbol{y}_{1}, \boldsymbol{y}_{2}, \ldots, \boldsymbol{y}_{n}\right)=\prod_{i=1}^{n} P\left(\boldsymbol{Y}_{i}=\boldsymbol{y}_{i} \mid \boldsymbol{\beta}, \boldsymbol{\Gamma}, \boldsymbol{D}\right),
$$

em que

$$
\begin{aligned}
& P\left(\boldsymbol{Y}_{i}=\boldsymbol{y}_{i} \mid \boldsymbol{\beta}, \boldsymbol{\Sigma}, \boldsymbol{D}\right)= \\
& \quad \int_{A_{i J}} \ldots \int_{A_{i 1}}\left[\int_{0}^{\infty}\left(\int_{(0, \infty)^{J}} \phi_{J}\left(\boldsymbol{z} \mid \boldsymbol{X}_{i} \boldsymbol{\beta}+\boldsymbol{D} \boldsymbol{w}, \kappa(\eta) \boldsymbol{\Sigma}\right) \phi_{J}(\boldsymbol{w} \mid 0, \kappa(\eta) \boldsymbol{I}) d \boldsymbol{w}\right) d H(\eta)\right] d \boldsymbol{z}
\end{aligned}
$$

com

$$
B_{i j}= \begin{cases}(0,+\infty) & \text { se } y_{i j}=1 \\ (-\infty, 0] & \text { se } y_{i j}=0\end{cases}
$$

Os parâmetros $\boldsymbol{\beta}$ e $\boldsymbol{D}$ são os mesmos definidos anteriormente em (3.1) e, para tornar o modelo identificável a matriz $\boldsymbol{\Sigma}$ é considerada uma matriz de correlações, ou seja, todos os componentes da diagonal principal da matriz $\boldsymbol{\Sigma}$ são iguais a 1 (um) e as demais são menores que um em módulo. Através da representação do modelo MENA dado em (3.10) pode-se observar que o modelo MENA 
pode ser representado usando variáveis latentes da seguinte forma

$$
P\left(\boldsymbol{Y}_{i}=\boldsymbol{y}_{i} \mid \boldsymbol{\beta}, \boldsymbol{\Sigma}, \boldsymbol{D}\right)=P\left(\boldsymbol{z}_{i} \in B_{i} \mid \boldsymbol{\beta}, \boldsymbol{\Sigma}, \boldsymbol{D}\right),
$$

em que $B_{i}=B_{i 1} \times B_{i 2} \times \cdots B_{i J} \mathrm{e}$

$$
\begin{aligned}
\boldsymbol{z}_{i} & =\boldsymbol{X}_{i} \boldsymbol{\beta}+\boldsymbol{D} \boldsymbol{w}_{i}+\boldsymbol{\epsilon}_{i} \\
\boldsymbol{\epsilon}_{i} & \sim \mathcal{N}_{J}\left(\mathbf{0}, \kappa\left(\eta_{i}\right) \boldsymbol{\Sigma}\right) \\
\boldsymbol{w}_{i} & \sim \mathcal{N}_{J}^{+}\left(\mathbf{0}, \kappa\left(\eta_{i}\right) \boldsymbol{I}\right) \\
\eta_{i} & \sim H\left(\eta_{i}\right) .
\end{aligned}
$$

Note que para $\kappa$ fixo, esta é a representação estocástica de uma variável normal assimétrica multivariada proposta por Sahu, Dey e Branco (2003). Esta representação nos permite realizar inferências utilizando a abordagem proposta por Albert e Chib (1993) para estimar os parâmetros de interesse em modelos binários através de variáveis latentes. Esta abordagem com dados aumentados foi utilizada por Chib e Greenberg (1998) e Chen e Dey (2003) para realização de inferências em seus modelos binários propostos. No processo de estimação, nós usaremos esquemas de Monte Carlo via Cadeias de Markov (MCMC), em particular, o algoritmo de Gibbs, para amostrar da distribuição a posteriori dos parâmetros de interesse.

Considerando que a matriz $\boldsymbol{D}$ é fixa e conhecida, temos que a distribuição conjunta a posteriori dos parâmetros de interesse e das variáveis latentes é então dada por

$$
\pi(\boldsymbol{\beta}, \boldsymbol{\Sigma}, \boldsymbol{z}, \boldsymbol{w}, \boldsymbol{\eta} \mid \boldsymbol{y}, \boldsymbol{D}) \propto L\left(\boldsymbol{\beta}, \boldsymbol{\Gamma} ; \boldsymbol{y}_{1}, \boldsymbol{y}_{2}, \ldots, \boldsymbol{y}_{n}\right) \pi(\boldsymbol{\beta}, \boldsymbol{\Sigma})
$$

em que $\pi(\boldsymbol{\beta}, \boldsymbol{\Sigma})$ é a distribuição conjunta a priori dos parâmetros $\boldsymbol{\beta}$ e $\boldsymbol{\Sigma}$. Usando a representação hieráquica do modelo MENA definida em de (3.11), temos esta distribuição a posteriori pode ser escrita da seguinte forma:

$$
\begin{aligned}
\pi(\boldsymbol{\beta}, \boldsymbol{\Sigma}, \boldsymbol{z}, \boldsymbol{w}, \boldsymbol{\eta} \mid \boldsymbol{y}, \boldsymbol{D}) \propto & \prod_{i=1}^{n} \phi\left(\boldsymbol{z} \mid \boldsymbol{X}_{i} \boldsymbol{\beta}+\boldsymbol{D} \boldsymbol{w}_{i}, \kappa\left(\eta_{i}\right) \boldsymbol{\Sigma}\right) \phi^{+}\left(\boldsymbol{w}_{i} \mid 0, \kappa\left(\eta_{i}\right) \boldsymbol{I}\right) h\left(\eta_{i}\right) \\
& \times \pi(\boldsymbol{\beta}, \boldsymbol{\Sigma}) \prod_{i=1}^{n} I\left(\boldsymbol{z}_{i} \in B_{i}\right) \prod_{j=1}^{J} I\left(w_{i j}>0\right) .
\end{aligned}
$$

Note que no caso em que a distribuição de mistura $H(\eta)$ depende de parâmetros desconhecidos, uma distribuição a priori para estes parâmetros adicionais deve ser especificada. O vetor de parâmetros livres de $\boldsymbol{\Sigma}$ é denotado por $\boldsymbol{\sigma}=\left(\sigma_{12}, \sigma_{13}, \ldots, \sigma_{J-1, J}\right)^{\prime} \in(-1,1)^{p}$, com $p=J(J-1) / 2$ denotado a quantidade de parâmetros livres.

Assumindo que a priori $\boldsymbol{\beta}$ e $\boldsymbol{\Sigma}$ são independentes, temos que a distribuição a posteriori dos parâmetros de interesse e das variáveis latentes condicionadas as variáveis observadas $\boldsymbol{y}$ e o matriz 
$D$ é

$$
\begin{aligned}
& \pi(\boldsymbol{\beta}, \boldsymbol{\Sigma}, \boldsymbol{\eta}, \boldsymbol{z}, \boldsymbol{w} \mid \boldsymbol{y}, \boldsymbol{D}) \propto \pi(\boldsymbol{\beta}) \pi(\boldsymbol{\Sigma}) \times|\boldsymbol{\Sigma}|^{-n / 2} \prod_{i=1}^{n}\left[\kappa\left(\eta_{i}\right)\right]^{-J} \\
& \quad \times \exp \left[-\frac{1}{2} \sum_{i=1}^{n} \frac{1}{\kappa\left(\eta_{i}\right)}\left(\boldsymbol{z}_{i}-\boldsymbol{X}_{i} \boldsymbol{\beta}-\boldsymbol{D} \boldsymbol{w}_{i}\right)^{\prime} \boldsymbol{\Sigma}^{-1}\left(\boldsymbol{z}_{i}-\boldsymbol{X}_{i} \boldsymbol{\beta}-\boldsymbol{D} \boldsymbol{w}_{i}\right)\right] \prod_{i=1}^{n} I\left(\boldsymbol{z}_{i} \in B_{i}\right) \\
& \quad \times \exp \left[-\frac{1}{2} \sum_{i=1}^{n} \frac{1}{\kappa\left(\eta_{i}\right)} \boldsymbol{w}_{i}^{\prime} \boldsymbol{w}_{i}\right] \prod_{i=1}^{n}\left(\prod_{j=1}^{J} I\left(w_{i j}>0\right)\right) \prod_{i=1}^{n} h\left(\eta_{i}\right) .
\end{aligned}
$$

Temos, devido a representação estocástica de Sahu, Dey e Branco (2003) e a abordagem de dados aumentados, que as distribuições condicionais completas das variáveis latentes $\boldsymbol{z}$ e $\boldsymbol{w}$ são conhecidas. Isto facilita muito a implementação do algoritmo amostrador de Gibbs. As distribuições condicionais completas de $\boldsymbol{\beta}, \boldsymbol{\Sigma}, \boldsymbol{\eta}, \boldsymbol{z}$ e $\boldsymbol{w}$ são dadas por

$$
\begin{aligned}
& {\left[\boldsymbol{Z}_{i} \mid \boldsymbol{w}, \boldsymbol{\beta}, \boldsymbol{D}, \boldsymbol{\Sigma}, \boldsymbol{\eta}, \boldsymbol{y}\right] \sim \mathcal{N}_{J}\left(\boldsymbol{X}_{i} \boldsymbol{\beta}-\boldsymbol{D} \boldsymbol{w}_{i}, \kappa\left(\eta_{i}\right) \boldsymbol{\Sigma}\right) I\left(\boldsymbol{Z}_{i} \in B_{i}\right),} \\
& {\left[\boldsymbol{W}_{i} \mid \boldsymbol{\beta}, \boldsymbol{D}, \boldsymbol{z}, \boldsymbol{\Sigma}, \boldsymbol{\eta}\right] \sim \mathcal{N}_{J}\left(\boldsymbol{\mu}_{\boldsymbol{z}_{i}}, \boldsymbol{\Sigma}_{\boldsymbol{z}_{i}}\right) \prod_{j=1}^{J} I\left(w_{i j}>0\right),} \\
& {\left[\eta_{i} \mid \boldsymbol{\beta}, \boldsymbol{D}, \boldsymbol{z}, \boldsymbol{w}, \boldsymbol{\Sigma}\right] \propto\left[\kappa\left(\eta_{i}\right)\right]^{-J / 2} \exp \left(-\frac{r^{2}}{2 \kappa\left(\eta_{i}\right)}\right) h\left(\eta_{i}\right),} \\
& {[\boldsymbol{\beta} \mid \boldsymbol{z}, \boldsymbol{w}, \boldsymbol{D}, \boldsymbol{\Sigma}, \boldsymbol{\eta}] \propto \exp \left[-\frac{1}{2}\left(\boldsymbol{\beta}-\boldsymbol{\beta}_{1}\right)^{\prime} \boldsymbol{B}_{1}^{-1}\left(\boldsymbol{\beta}+\boldsymbol{\beta}_{1}\right)\right] \pi(\boldsymbol{\beta}),} \\
& {[\boldsymbol{\Sigma} \mid \boldsymbol{\beta}, \boldsymbol{D}, \boldsymbol{z}, \boldsymbol{w}, \boldsymbol{\eta}] \propto|\boldsymbol{\Sigma}|^{-n / 2} \exp \left[-\frac{1}{2} \sum_{i=1}^{n} \frac{1}{\kappa\left(\eta_{i}\right)} r_{i}^{2}\right] \pi(\boldsymbol{\Sigma}),}
\end{aligned}
$$

em que

$$
\begin{aligned}
& r_{i}^{2}=\left(\boldsymbol{z}_{i}-\boldsymbol{X}_{i} \boldsymbol{\beta}-\boldsymbol{D} \boldsymbol{w}_{i}\right)^{\prime} \boldsymbol{\Sigma}^{-1}\left(\boldsymbol{z}_{i}-\boldsymbol{X}_{i} \boldsymbol{\beta}-\boldsymbol{D} \boldsymbol{w}_{i}\right), \\
& \boldsymbol{B}_{1}=\left[\sum_{i=1}^{n} \frac{1}{\kappa\left(\eta_{i}\right)} \boldsymbol{X}_{i}^{\top} \boldsymbol{\Sigma}^{-1} \boldsymbol{X}_{i}\right]^{-1}, \boldsymbol{\beta}_{1}=\boldsymbol{B}_{1} \sum_{i=1}^{n} \frac{1}{\kappa\left(\eta_{i}\right)} \boldsymbol{X}_{i}^{\top} \boldsymbol{\Sigma}^{-1}\left(\boldsymbol{z}_{i}-\boldsymbol{D} \boldsymbol{w}_{i}\right), \\
& \boldsymbol{\Sigma}_{\boldsymbol{w}_{i}}=\kappa\left(\eta_{i}\right)\left[\boldsymbol{D}^{\prime} \boldsymbol{\Sigma}^{-1} \boldsymbol{D}+\mathrm{I}\right]^{-1} \text { e } \boldsymbol{\mu}_{\boldsymbol{w}_{i}}=-\left[\kappa\left(\eta_{i}\right)\right]^{-1} \boldsymbol{\Sigma}_{\boldsymbol{w}_{i}} \boldsymbol{D}^{\prime} \boldsymbol{\Sigma}^{-1}\left(\boldsymbol{z}_{i}+\boldsymbol{X}_{i} \boldsymbol{\beta}\right) .
\end{aligned}
$$

Observe que as distribuições condicionais completas (3.13) e (3.14) são normais $J$-variadas truncadas. Por não ser trivial amostrar da distribuição normal multivariada truncada, nós utilizamos o amostrador de Gibbs novamente nesta etapa do processo de simulação. Temos, após algumas operações matriciais, que as distribuições condicionais completas de $\boldsymbol{z}_{i}$ são dadas por

$$
Z_{i j} \mid\left\{Z_{i k} ; k \neq j\right\}, \boldsymbol{y}, \boldsymbol{w}, \boldsymbol{\beta}, \boldsymbol{D}, \boldsymbol{\Sigma}, \eta \sim \mathcal{N}\left(m_{i j}, \frac{v_{j j}}{\kappa\left(\eta_{i}\right)}\right),
$$

em que

$$
\begin{aligned}
m_{i j} & =\boldsymbol{x}_{i j}^{\prime \top} \boldsymbol{\beta}+\delta_{j} w_{i j}+\frac{\kappa\left(\eta_{i}\right)}{v_{j j}} \sum_{l=1, l \neq j}^{n}\left[z_{i l}-\left(x_{i l}^{\top} \boldsymbol{\beta}_{l}+\delta_{l} w_{i l}\right)\right] \\
v_{j l} & =\left\{\boldsymbol{\Sigma}^{-1}\right\}_{j l},
\end{aligned}
$$


$\operatorname{com}\left\{\boldsymbol{\Sigma}^{-1}\right\}_{j l}$ denotando o $l$-ésimo elemento da $j$-ésima linha da matriz $\boldsymbol{\Sigma}^{-1}$. As distribuições $a$ priori de $\boldsymbol{\beta}$ e $\boldsymbol{\Sigma}$ são espeficadas nas próximas seções.

\subsection{Distribuição condicional completa dos coeficientes da regressão}

Desde que cada coeficiente da regressão apresenta variação em toda a reta dos números reais e a distribuição condicional de $\boldsymbol{\beta}$ dada em (3.16) é proporcional ao produto da distribuição a priori de $\boldsymbol{\beta}$ pelo núcleo de uma distribuição normal multivariada, temos que a distribuição condicional completa conjugada de $\boldsymbol{\beta}$ é uma distribuição normal multivariada quando se assume uma distribuição a priori normal multivariada para $\boldsymbol{\beta}$. A distribuição condicional completa de $\boldsymbol{\beta}$ é então dada por

$$
\boldsymbol{\beta} \mid \boldsymbol{z}, \boldsymbol{w}, \boldsymbol{D}, \boldsymbol{\Sigma}, \boldsymbol{\eta} \sim \mathcal{N}_{k}\left(\boldsymbol{\beta}_{1}, \boldsymbol{B}_{1}\right)
$$

em que a distribuição a priori dos coeficientes da regressão é normal multivariada com vetor de médias $\boldsymbol{\beta}_{0}$ e matriz de covariâncias $\boldsymbol{B}_{0}$. O vetor de médias e matriz de covariâncias da distribuição condicional completa de $\boldsymbol{\beta}$ são, respectivamente, dados por

$$
\boldsymbol{B}_{1}=\left[\boldsymbol{B}_{0}^{-1}+\sum_{i=1}^{n} \frac{1}{\kappa\left(\eta_{i}\right)} \boldsymbol{X}_{i}^{\top} \boldsymbol{\Sigma}^{-1} \boldsymbol{X}_{i}\right]^{-1} \text { e } \boldsymbol{\beta}_{1}=\boldsymbol{B}_{1}\left[\boldsymbol{B}_{0}^{-1} \boldsymbol{\beta}_{0}+\sum_{i=1}^{n} \frac{1}{\kappa\left(\eta_{i}\right)} \boldsymbol{X}_{i}^{\top} \boldsymbol{\Sigma}^{-1}\left(\boldsymbol{z}_{i}-\boldsymbol{D} \boldsymbol{w}_{i}\right)\right]
$$

Esta especificação de distribuição a priori normal para $\boldsymbol{\beta}$ fornece uma distribuição condicional completa conhecida e de fácil simulação.

\subsection{Distribuição condicional completa da matriz de dependência}

Pela Seção 3.1, sabemos que devemos considerar algumas restrições no espaço paramétrico do modelo MENA para garantir a identificabilidade do modelo. A restrição considerada aqui é que a matriz de dispersão da variável latente que induz a função de ligação MENA é da forma

$$
\boldsymbol{\Sigma}=\left(\begin{array}{ccccc}
1 & \sigma_{12} & \sigma_{13} & \cdots & \sigma_{1 J} \\
\sigma_{21} & 1 & \sigma_{23} & \cdots & \sigma_{2 J} \\
\vdots & \cdots & \ddots & \cdots & \vdots \\
\sigma_{J-1,1} & \cdots & \sigma_{J-1, J-2} & 1 & \sigma_{J-1, J} \\
\sigma_{J 1} & \cdots & \sigma_{J, J-2} & \sigma_{J, J-1} & 1
\end{array}\right)
$$

em que $\left|\sigma_{j j^{\prime}}\right| \leq 1\left(j \neq j^{\prime} ; j, j^{\prime}=1, \ldots, J\right)$. Esta é a mesma matriz de dispersão considerada por Chib e Greenberg (1998) e Chen e Dey (2003) no processo de estimação nos modelos multivariados com funções de ligação probito e MEN, respectivamente. Chib e Greenberg (1998) estimam os parâmetros de interesse do modelo multivariado probito através de máxima verossimilhança e inferência bayesiana, utilizando, respectivamente, o algoritmo EM e algoritmos de Monte Carlo via Cadeias de Markov. No entanto, Chen e Dey (1998) utilizam apenas inferência bayesiana na estimação dos parâmetros de interesse dos modelos de mistura de escala normal. No contexto bayesiano, os autores consideram distribuições a priori uniformes marginalmente independentes no intervalo $[-1 ; 1]$ para os parâmetros $\sigma_{j j^{\prime}}$ dados em (3.22). Estes autores utilizaram o algoritmo de Metropolis-Hastings 
com densidade proposta normal truncada no intervalo [-1;1]. Barnard et al. (2000) usa a decomposição $\boldsymbol{\Omega}=\boldsymbol{Q} \boldsymbol{\Sigma} \boldsymbol{Q}$, e assume distribuições a priori independentes para $\boldsymbol{\Sigma}$ e $\boldsymbol{Q}$. No entanto, Tabet (2007) mostra que estas abordagens não são eficientes em dimensões elevadas e propõe usar uma expansão paramétrica para dados aumentados (Liu e Wu, 1999).

\subsubsection{Expansão paramétrica para dados aumentados}

A expansão paramétrica para dados aumentados (PX-DA) foi introduzida por Liu e Wu (1999). O PX-DA é uma técnica utilizada, geralmente, para acelerar a convergência de cadeias de Markov. O método baseia-se em duas etapas:

- Etapa 1: define-se um parâmetro adicional (oculto), $\boldsymbol{\alpha}$, no modelo com os dados completos (dados aumentados), modelo denotado aqui por $\pi(\boldsymbol{y}, \boldsymbol{z} \mid \boldsymbol{\beta}, \boldsymbol{\Sigma}, \boldsymbol{D})$.

- Etapa 2: expande-se o espaço paramétrico do modelo com dados aumentados para um modelo maior (expandido), modelo denotado aqui por $\pi\left(\boldsymbol{y}, \boldsymbol{z}^{*} \mid \boldsymbol{\beta}, \boldsymbol{\Sigma}, \boldsymbol{D}, \boldsymbol{\alpha}\right)$. Este modelo expandido deve preservar a distribuição do modelo de dados observados. Isto é, a seguinte igualdade deve ser satisfeita:

$$
\int \pi\left(\boldsymbol{y}, \boldsymbol{z}^{*} \mid \boldsymbol{\beta}, \boldsymbol{\Sigma}, \boldsymbol{D}, \boldsymbol{\alpha}\right) d \boldsymbol{z}^{*}=\pi(\boldsymbol{y} \mid \boldsymbol{\beta}, \boldsymbol{\Sigma}, \boldsymbol{D}) .
$$

em que $\boldsymbol{\alpha}$ é um parâmetro associado a variável latente $\boldsymbol{z}^{*}$.

Além disto, para implementar a metodologia dos dados aumentados nesta configuração, a distribuição conjunta a priori do parâmetro $\boldsymbol{\alpha}$ e a distribuição conjunta a priori dos parâmetros originais de interesse, $(\boldsymbol{\beta}, \boldsymbol{\Sigma}, \boldsymbol{D})$, precisam ser especificadas de tal forma que a distribuição a priori de $\boldsymbol{\alpha}$ deve ser a mesma para o modelo original e para o modelo expandido. Isto é,

$$
\int \pi(\boldsymbol{\beta}, \boldsymbol{\Sigma}, \boldsymbol{D}, \boldsymbol{\alpha}) d \boldsymbol{\alpha}=\pi(\boldsymbol{\beta}, \boldsymbol{\Sigma}, \boldsymbol{D})
$$

Esta relação é garantida fazendo $\pi(\boldsymbol{\beta}, \boldsymbol{\Sigma}, \boldsymbol{D}, \boldsymbol{\alpha})=\pi(\boldsymbol{\beta}, \boldsymbol{\Sigma}, \boldsymbol{D}) \pi(\boldsymbol{\alpha})$. A seguir é apresenta as etapas do algoritmo PX-DA.

\section{Algoritmo PX-DA}

- Etapa 1: Gera-se o vetor $(\boldsymbol{\alpha}, \boldsymbol{z})$ conjuntamente amostrando de

$$
\begin{aligned}
\boldsymbol{\alpha} & \sim \pi(\boldsymbol{\alpha} \mid \boldsymbol{z}) \\
\boldsymbol{z} & \sim \pi(\boldsymbol{z} \mid \boldsymbol{\alpha}, \boldsymbol{\Sigma}, \boldsymbol{y}) \propto \pi(\boldsymbol{z}, \boldsymbol{y} \mid \boldsymbol{\alpha}, \boldsymbol{\Sigma})
\end{aligned}
$$

- Etapa 2: Gera-se $(\boldsymbol{\alpha}, \boldsymbol{\Sigma})$ conjuntamente amostrando de

$$
[\boldsymbol{\alpha}, \boldsymbol{\Sigma} \mid \boldsymbol{z}, \boldsymbol{y}] \sim \pi(\boldsymbol{z}, \boldsymbol{y} \mid \boldsymbol{\alpha}, \boldsymbol{\Sigma}) \pi(\boldsymbol{\alpha} \mid \boldsymbol{z}) \pi(\boldsymbol{\Sigma})
$$


As etapas para a construção de um algoritmo PX-DA para amostrar da distribuição condicional completa de $\boldsymbol{\Sigma}$ definida em (3.17) é como segue.

\section{Etapa 1: Definição do modelo expandido}

Da expressão (3.17), temos que o modelo com dados aumentados é dado por

$$
\pi(\boldsymbol{y}, \boldsymbol{z} \mid \boldsymbol{w}, \boldsymbol{\Sigma}, \boldsymbol{\beta}, \boldsymbol{\eta}, \boldsymbol{D}) \propto \prod_{i=1}^{n} \phi_{J}\left(\boldsymbol{z} \mid \boldsymbol{X}_{i} \boldsymbol{\beta}+\boldsymbol{D} \boldsymbol{w}_{i}, \kappa\left(\eta_{i}\right) \boldsymbol{\Sigma}\right) I\left(\boldsymbol{z}_{i} \in B_{i}\right),
$$

em que $B_{i}=B_{i 1} \times B_{i 2} \times \cdots B_{i J}$ é definido em (3.11) e $\boldsymbol{w}, \boldsymbol{\Sigma}, \boldsymbol{\beta}, \boldsymbol{\eta}_{i}$ e $\boldsymbol{D}$ são quantidades fixas.

\section{Etapa 2: Definição da expansão paramétrica e a da transformação de variáveis}

Liu e Wu (1999) argumenta que sob certas condições, o vetor de parâmetros ocultos $\boldsymbol{\alpha}$ pode ser induzido por uma transformação nos dados. Esta transformação, denotada aqui por $\boldsymbol{z}=T_{\boldsymbol{\alpha}}\left(\boldsymbol{z}^{*}\right)$, precisa ser definida de tal modo que para qualquer valor fixo de $\boldsymbol{\alpha}, T_{\boldsymbol{\alpha}}(\boldsymbol{z})$ deve ser uma função um a um e diferenciável entre $\boldsymbol{z}^{*}$ e $\boldsymbol{z}$. A transformação será definida por

$$
\pi\left(\boldsymbol{y}, \boldsymbol{z}^{*} \mid \boldsymbol{w}, \boldsymbol{\Sigma}, \boldsymbol{\beta}, \boldsymbol{\eta}, \boldsymbol{D}\right)=\pi\left(\boldsymbol{y}, T_{\boldsymbol{\alpha}}\left(\boldsymbol{z}^{*}\right) \mid \boldsymbol{w}, \boldsymbol{\Sigma}, \boldsymbol{\beta}, \boldsymbol{\eta}, \boldsymbol{D}\right)\left|J: \boldsymbol{z} \rightarrow \boldsymbol{z}^{*}\right|
$$

em que $\left|J: \boldsymbol{z} \rightarrow \boldsymbol{z}^{*}\right|$ denota o determinante do jacobiano da transformação $T_{\boldsymbol{\alpha}}$ avaliada em $\boldsymbol{z}^{*}$ e

$$
\boldsymbol{z}=T_{\boldsymbol{\alpha}}\left(\boldsymbol{z}^{*}\right)=\boldsymbol{Q}^{-1} \boldsymbol{z}^{*}
$$

em que $\boldsymbol{Q}$ é uma matriz diagonal com elementos positivos dados por $q_{j j}=\sqrt{\sigma_{j j}}$, para $j=1 \ldots, J$. É importante notar que a matriz de parâmetros $\boldsymbol{Q}$ é identificável somente para o modelo expandido. Assim como em Tabet (2007), nós iremos escolher $\boldsymbol{\alpha}=\left(\alpha_{1}, \alpha_{2}, \ldots, \alpha_{J}\right)^{\prime}$ uma função de $\boldsymbol{Q}$ tal que

$$
\alpha_{j}=\frac{\Sigma^{j j}}{2 q_{j j}^{2}}
$$

com $\boldsymbol{\Sigma}^{j j}$ denotando o $j$-ésimo elemento da diagonal da matriz $\boldsymbol{\Sigma}^{-1}$. Neste caso, para qualquer valor fixo do vetor $\boldsymbol{\alpha}$, a matriz $\boldsymbol{Q}$ é função um a um de $\boldsymbol{\alpha}$ e $T_{\boldsymbol{\alpha}}(\boldsymbol{z})$, diferenciável entre $\boldsymbol{z}$ e $\boldsymbol{z}^{*}$. A escolha do vetor $\boldsymbol{\alpha}$ na expressão (3.26) não foi arbitrária. Tabet (2007) mostra que esta escolha para $\boldsymbol{\alpha}$, combinada com a distribuição a priori para $\boldsymbol{\Sigma}$ proposta por Barnard et al. (2000), resulta em uma distribuição a posteriori fácil de amostrar.

\section{Etapa 3: O modelo expandido com dados transformados}

Considerando a transformação de variáveis em (3.25) no modelo expandido dado em (3.23), temos que o modelo expandido com os dados transformados é

$$
\pi\left(\boldsymbol{y}, \boldsymbol{z}^{*} \mid \boldsymbol{w}, \boldsymbol{\Sigma}, \boldsymbol{\beta}, \boldsymbol{\eta}, \boldsymbol{D}\right) \propto \prod_{i=1}^{n} \phi_{J}\left(\boldsymbol{Q}^{-1} \boldsymbol{z}_{i}^{*} \mid \boldsymbol{X}_{i} \boldsymbol{\beta}+\boldsymbol{D} \boldsymbol{w}_{i}, \kappa\left(\eta_{i}\right) \boldsymbol{\Sigma}\right) I\left(\boldsymbol{Q}^{-1} \boldsymbol{z}^{*} \in B_{i}\right)\left|J: \boldsymbol{z} \rightarrow \boldsymbol{z}_{i}^{*}\right|,
$$


em que

$$
\left|J: \boldsymbol{z} \rightarrow \boldsymbol{z}^{*}\right|=\left|\frac{d \boldsymbol{z}}{d \boldsymbol{z}^{*}}\right|=\left|\boldsymbol{I}_{n} \otimes \boldsymbol{Q}\right|=|\boldsymbol{Q}|^{-n},
$$

é o determinante do jacobiano da transformação (3.25) e $B_{i}=B_{i 1} \times B_{i 2} \times \cdots B_{i J}$ é definido em (3.11). Após realizar algumas operações matricias, temos que

$$
\begin{aligned}
\pi\left(\boldsymbol{y}, \boldsymbol{z}^{*}\right. & \mid \boldsymbol{w}, \boldsymbol{\beta}, \boldsymbol{\delta}, \boldsymbol{\Sigma}, \eta) \propto|\boldsymbol{Q} \boldsymbol{\Sigma} \boldsymbol{Q}|^{-n / 2} \prod_{i=1}^{n} I\left(\boldsymbol{z}_{i}^{*} \in B_{i}\right) \\
& \times \exp \left\{-\sum_{i=1}^{n} \frac{1}{2 \kappa\left(\eta_{i}\right)}\left[\boldsymbol{z}_{i}^{*}-\boldsymbol{Q}\left(\boldsymbol{X}_{i} \boldsymbol{\beta}+\boldsymbol{D} \boldsymbol{w}_{i}\right)\right]^{\prime}(\boldsymbol{Q} \boldsymbol{\Sigma} \boldsymbol{Q})^{-1}\left[\boldsymbol{z}_{i}^{*}-\boldsymbol{Q}\left(\boldsymbol{X}_{i} \boldsymbol{\beta}+\boldsymbol{D} \boldsymbol{w}_{i}\right)\right]\right\}
\end{aligned}
$$

e a verossimilhança do modelo expandido apresentado pode ser expressa como

$$
\pi\left(\boldsymbol{y}, \boldsymbol{z}^{*} \mid \boldsymbol{w}, \boldsymbol{\beta}, \delta, \boldsymbol{\Sigma}, \eta\right) \propto|\boldsymbol{\Sigma}| \exp \left(\operatorname{tr}\left\{\boldsymbol{\Sigma}^{-1} \boldsymbol{\epsilon}^{\prime} \boldsymbol{\epsilon}\right\}\right) \prod_{i=1}^{n} I\left(\boldsymbol{z}_{i}^{*} \in B_{i}\right)
$$

em que $\boldsymbol{W}=\left(\operatorname{diag}\left(w_{1}\right), \ldots, \operatorname{diag}\left(w_{n}\right)\right)^{\prime}, \boldsymbol{\delta}=\left(\delta_{1}, \ldots, \delta_{J}\right)$

$$
\boldsymbol{\Omega}=\boldsymbol{Q} \boldsymbol{\Sigma} \boldsymbol{Q} \text { е } \boldsymbol{\epsilon}=\frac{1}{\sqrt{\kappa(\eta)}} \boldsymbol{Q}(\boldsymbol{z}-\boldsymbol{X} \boldsymbol{\beta}+\boldsymbol{W} \boldsymbol{\delta})
$$

e $\operatorname{tr}(A)$ denota a função traço da matriz $A$.

\section{Etapa 4: A distribuição a priori}

Barnard et al. (2000) mostrou que sob a transformação $\boldsymbol{\Omega}=\boldsymbol{Q} \boldsymbol{\Sigma} \boldsymbol{Q}$, a distribuição de $\boldsymbol{\Omega}$ é uma distribuição Wishart inversa da seguinte forma:

$$
\pi(\boldsymbol{\Omega})=\pi(\boldsymbol{\alpha}, \boldsymbol{\Sigma})|J: \boldsymbol{\Omega} \rightarrow(\boldsymbol{Q}, \boldsymbol{\Sigma})|=\pi(\boldsymbol{\Sigma}) \pi(\boldsymbol{\alpha} \mid \boldsymbol{\Sigma})
$$

em que a distribuição a priori da matriz de correlações $\boldsymbol{\Sigma}$ é dada por

$$
\pi(\boldsymbol{\Sigma}) \propto|\boldsymbol{\Sigma}|^{\frac{J(J-1)}{2}-1}\left(\prod_{j=1}^{J}\left|\boldsymbol{\Sigma}_{j j}\right|\right)^{-(J+1) / 2},
$$

com $\boldsymbol{\Sigma}_{j j}$ denotando a principal submatriz de $\boldsymbol{\Sigma}$. Esta distribuição conjunta retorna distribuições marginalmente distribuídos com uma distribuição uniforme sobre o intervalo $[-1,1]$. No entanto, as distribuições marginais não são independentes. Tabet (2007) assume que as distribuições marginais de $\boldsymbol{\alpha} \mid \boldsymbol{\Sigma}$ são distribuições gamas independentes com parâmetro de forma $(J+1) / 2$ e parâmetro de escala 1 (um).

$$
\pi(\boldsymbol{\alpha} \mid \boldsymbol{\Sigma})=\pi(\boldsymbol{\alpha})=\prod_{j=1}^{J} \frac{1}{\Gamma((J-1) / 2)} \alpha_{j}^{-(J-1) / 2} e^{-\alpha_{j}} .
$$

Valores de $(\boldsymbol{\alpha}, \boldsymbol{\Sigma})$ podem ser gerados amostrando de $\boldsymbol{\Omega}$ da distribuição Wishart inversa e transformando os dados usando $\boldsymbol{\Omega}=\boldsymbol{Q} \boldsymbol{\Sigma} \boldsymbol{Q}$. Note que a distribuição a priori de $\boldsymbol{\Sigma}$ é a mesma no modelo expandido e no modelo com dados observados. 


\section{Etapa 5: A distribuição a posteriori}

A distribuição a posteriori conjunta de $(\boldsymbol{\alpha}, \boldsymbol{\Sigma})$ é dada pelo produto do modelo expandido (3.27) com as distribuições a priori de $\boldsymbol{\Sigma}$ e $\boldsymbol{\alpha}$ dadas, respectivamente, em (3.30) e (3.31).

$$
\begin{aligned}
& \pi\left(\boldsymbol{\Sigma}, \alpha \mid \boldsymbol{\beta}, \boldsymbol{\delta}, \boldsymbol{y}, \boldsymbol{z}^{*}, \boldsymbol{w}, \eta\right) \propto|\boldsymbol{\Sigma}| \exp \left(\operatorname{tr}\left\{\boldsymbol{\Sigma}^{-1} \boldsymbol{\epsilon}^{\prime} \boldsymbol{\epsilon}\right\}\right) \\
& \quad \times|\boldsymbol{\Sigma}|^{\frac{J(J-1)}{2}-1}\left(\prod_{j=1}^{J}\left|\boldsymbol{\Sigma}_{j j}\right|\right)^{-(J+1) / 2} \times \prod_{j=1}^{J} \alpha_{j}^{-(J-1) / 2} e^{-\alpha_{j}} .
\end{aligned}
$$

Escrevendo esta distribuição em função de $\boldsymbol{\Omega}=\boldsymbol{Q} \boldsymbol{\Sigma} \boldsymbol{Q}$, nós temos que

$$
\pi\left(\boldsymbol{\Sigma} \mid \boldsymbol{\beta}, \boldsymbol{\delta}, \boldsymbol{y}, \boldsymbol{z}^{*}, \boldsymbol{w}, \eta\right) \propto|\boldsymbol{\Omega}| \exp \left(\operatorname{tr}\left\{\boldsymbol{\Omega}^{-1}\left(\boldsymbol{\epsilon}^{*}\right)^{\prime} \boldsymbol{\epsilon}\right\}\right) .
$$

Esta é a distribuição Wishart inversa com $\nu=n+J+1$ graus de liberdade e parâmetro $S=\epsilon^{\prime} \epsilon$. O algoritmo para amostrar de $\boldsymbol{\Sigma}, \boldsymbol{\alpha} \mid \boldsymbol{\beta}, \boldsymbol{\delta}, \boldsymbol{z}^{*}, \boldsymbol{w}, \boldsymbol{y}$ é dado a seguir:

\section{Algoritmo 3.1 Amostrar de $\boldsymbol{\Sigma}, \boldsymbol{\alpha} \mid \boldsymbol{\beta}, \boldsymbol{\delta}, \boldsymbol{z}^{*}, \boldsymbol{w}, \boldsymbol{y}$}

1. Amostre $\alpha_{j} \mid \boldsymbol{\Sigma}$ da distribuição gama, $\operatorname{Gama}\left(\frac{J+1}{2}, 1\right)$, para $j=1, \ldots, J$;

2. Calcule a matriz diagonal $Q$, em que cada elemento da diagonal $q_{j}=\sqrt{\frac{\boldsymbol{\Sigma}^{j j}}{2 \alpha_{j}}}$ e $\boldsymbol{\Sigma}^{j j}$ é o $i$-ésimo elemento da diagonal de $\boldsymbol{\Sigma}^{-1}$;

3. Calcule $\boldsymbol{z}^{*}=t_{\boldsymbol{\alpha}}(\boldsymbol{z})=\boldsymbol{Q} \boldsymbol{z}$ ou equivalentemente $\epsilon=\frac{1}{\sqrt{\kappa(\eta)}} \boldsymbol{Q}(\boldsymbol{z}-\boldsymbol{X} \boldsymbol{\beta}+w \delta)$;

4. Amostre $\boldsymbol{\Omega} \mid \boldsymbol{\beta}, \boldsymbol{\delta}, \boldsymbol{z}^{*}, \boldsymbol{w}, \boldsymbol{y}$ da distribuição Wishart inversa, $\boldsymbol{\Omega} \sim I W\left(n+J+1, \boldsymbol{\epsilon}^{\prime} \boldsymbol{\epsilon}\right)$;

5. Calcule $\boldsymbol{\Sigma}=Q^{-1} \Omega Q^{-1}$.

Para gerar da distribuição apresentada em (3.15), nós precisamos conhecer a forma da distribuição de mistura $H$. Nós apresentamos nas próximas seções alguns algoritmos para gerar destas distribuições em alguns casos especiais dos modelos de mistura de escala normal assimétrica.

\subsection{Distribuição condicional completa da variável de mistura}

Nesta seção mostramos como gerar amostras das distribuições condicionais completas das variáveis de mistura de alguns casos especiais de distribuições representáveis através de mistura de escala da distribuição normal assimétrica (MENA). Iniciamos com o modelo multivariado $t$ assimétrico, pois não é necessário gerar da variável de mistura $\eta$ no modelo multivariado probito assimétrico (MMPA). 


\section{Distribuição condicional completa do parâmetro de mistura no MMTA}

Combinando a função densidade da distribuição de mistura (2.35) com a distribuição a posteriori (3.15), temos que a distribuição condicional completa da variável de mistura no modelo multivariado $t$ assimétrico (MMTA) é definida da seguinte forma.

$$
\eta \mid \boldsymbol{\beta}, \boldsymbol{D}, \boldsymbol{z}, \boldsymbol{w}, \boldsymbol{\Sigma} \sim \operatorname{Gama}\left(\frac{\nu+J}{2}, \frac{1}{2}\left[\nu+\left(\boldsymbol{z}_{i}-\boldsymbol{X}_{i} \boldsymbol{\beta}-\boldsymbol{D} \boldsymbol{w}_{i}\right)^{\prime} \boldsymbol{\Sigma}^{-1}\left(\boldsymbol{z}_{i}-\boldsymbol{X}_{i} \boldsymbol{\beta}-\boldsymbol{D} \boldsymbol{w}_{i}\right)\right]\right),
$$

em que $\operatorname{Gama}(a, b)$ representa uma distribuição gama com parâmetro de forma $a>0$, parâmetro de escala $b>0$ e função de densidade de probabilidade

$$
f(\eta \mid a, b)=\frac{b^{a}}{\Gamma(a)} \eta^{a-1} e^{-b \eta}
$$

$\operatorname{com} \Gamma(a)=\int_{0}^{\infty} t^{a-1} e^{-t} d t$ denotanto a função gama avaliada no ponto $a$.

\section{Distribuição condicional completa do parâmetro de mistura no MMLA}

A distribuição condicional completa da variável de mistura no modelo multivariado logístico assimétrico (MMLA) é obtida combinando a distribuição a posteriori definida em (3.15) com distribuição de mistura (2.36) apresentada na Seção 2.2.1. Neste caso, temos que a distribuição condicional completa de $\eta$ é proporcional a

$$
\frac{1}{\left(\eta^{2}\right)^{J / 2}} \exp \left(-\frac{r^{2}}{8} \frac{1}{\eta^{2}}\right) \sum_{k=1}^{\infty}(-1)^{k+1} k^{2} \eta \exp \left\{-2 k^{2} \eta^{2}\right\},
$$

em que $r^{2}$ é forma quadrática definida em (3.18). Note que a distribuição (3.33) não é uma função de densidade de probabilidade conhecida. Logo, métodos de simulação mais sofisticados devem ser empreendidos para gerar valores desta distribuição.

Aqui é apresentado um algoritmo de Metropolis-Hastings similar ao desenvolvido Chen e Dey (1998) para o modelo multivarido logístico simétrico (MML).

Algoritmo 3.2 Amostrar de $\eta \mid \boldsymbol{\beta}, \boldsymbol{D}, \boldsymbol{z}, \boldsymbol{w}, \boldsymbol{\Sigma}$ no MMLA

1. Inicialize o contador de iterações $t=0$ e especifique um valor inicial para $\eta^{(0)}$;

2. Gere um novo valor para a variável de mistura $\eta^{*}$ através da seguinte distribuição proposta

$$
\eta^{* 2} \sim \mathcal{G I}\left(\frac{J+\nu}{2}, \frac{1}{8}\left[\left(\boldsymbol{z}_{i}-\boldsymbol{X}_{i} \boldsymbol{\beta}-\boldsymbol{D} \boldsymbol{w}_{i}\right)^{\prime} \boldsymbol{\Sigma}^{-1}\left(\boldsymbol{z}_{i}-\boldsymbol{X}_{i} \boldsymbol{\beta}-\boldsymbol{D} \boldsymbol{w}_{i}\right)+\frac{\nu}{b^{2}}\right]\right)
$$

em que $\mathcal{G} \mathcal{I}(\eta \mid u, v)$ denota uma distribuição gama inversa com função de densidade de probabilidade $\frac{v^{u}}{\Gamma(u) \eta^{u+1}} e^{-v / \eta}, \eta>0$. 
3. Gere $u \sim U(0,1)$ e calcule a probabilidade de aceitação $\gamma$ da seguinte forma

$$
\gamma=\min \left\{\frac{h\left(\eta^{*}\right) / g_{L}\left(\eta^{*} \mid \nu, b\right)}{h(\eta) / g_{L}(\eta \mid \nu, b)}, 1\right\}
$$

em que as funções de densidades de probabilidade $h(\eta)$ e $g_{L}(\eta \mid \nu, b)$ são dados, respectivamente, em (2.36) e (3.34). Chen e Dey (1998) sugerem usar $\nu=5$ e $b=0,712$.

4. Se $u \leq \gamma$ então aceite o novo valor e faça $\eta^{(t+1)}=\eta^{*}$, caso contrário rejeite e faça $\eta^{(t+1)}=\eta$.

5. Incremente o contador de $t$ para $t+1$ e volte ao passo 2 .

Uma forma alternativa de gerar valores da distribuição condicional completa dada em (3.33) é apresentada em Holmes e Held (2006). Os autores usam o método da rejeição com distribuição proposta Gaussiana inversa generalizada em sua abordagem.

\section{Distribuição condicional completa do parâmetro de mistura no MMSA}

A distribuição condicional completa da variável de mistura no modelo multivariado stable assimétrico (MMSA) é definida da seguinte forma.

$$
\pi(\eta \mid \boldsymbol{\beta}, \boldsymbol{D}, \boldsymbol{z}, \boldsymbol{w}, \boldsymbol{\Sigma}) \propto(2 \eta)^{-J / 2} \exp \left(-\frac{r^{2}}{4 \eta}\right) S_{p}(\eta \mid \alpha, 1),
$$

em que $S_{p}(\eta \mid \alpha, 1)$ é a distribuição stable dada em (2.38). A seguir é apresentado um algoritmo de Metropolis-Hastings similar ao desenvolvido Chen e Dey (1998) para o modelo multivarido stable assimétrico (MMSA).

Algoritmo 3.3 Amostrar de $\eta \mid \boldsymbol{\beta}, \boldsymbol{D}, \boldsymbol{z}, \boldsymbol{w}, \boldsymbol{\Sigma}$ no MMSA

1. Inicialize o contador de iterações $t=0$ e especifique um valor inicial para $\eta^{(0)}$;

2. Gere um novo valor para a variável de mistura $\eta^{*}$ através da seguinte distribuição proposta

$$
\eta^{*} \sim \mathcal{G I}\left(\frac{J+1}{2}, \frac{1}{4}\left[\left(\boldsymbol{z}_{i}-\boldsymbol{X}_{i} \boldsymbol{\beta}-\boldsymbol{D} \boldsymbol{w}_{i}\right)^{\prime} \boldsymbol{\Sigma}^{-1}\left(\boldsymbol{z}_{i}-\boldsymbol{X}_{i} \boldsymbol{\beta}-\boldsymbol{D} \boldsymbol{w}_{i}\right)+1\right]\right),
$$

em que $\mathcal{G I}(\eta \mid u, v)$ denota uma distribuição gama inversa.

3. Gere $u \sim U(0,1)$ e calcule a probabilidade de aceitação $\gamma$ da seguinte forma

$$
\gamma=\min \left\{\frac{h\left(\eta^{*} \mid \alpha, 1\right) / \mathcal{G I}\left(\eta^{*} \mid 1 / 2,1 / 4\right)}{h(\eta \mid \alpha, 1) / \mathcal{G I}(\eta \mid 1 / 2,1 / 4)}, 1\right\}
$$

Chen e Dey (1998) sugerem usar $\alpha$ entre 0,50 e 0,75.

4. Se $u \leq \gamma$ então aceite o novo valor e faça $\eta^{(t+1)}=\eta^{*}$, caso contrário rejeite e faça $\eta^{(t+1)}=\eta$. 
5. Incremente o contador de $t$ para $t+1$ e volte ao passo 2 .

\section{Distribuição condicional completa do parâmetro de mistura no MMEPA}

Similarmente aos modelos multivariados stable assimétricos, nós podemos utilizar um algoritmo de Metropolis para gerar amostras da distribuição de mistura no modelo multivariado exponencial potência assimétrico (MMEPA). Chen e Dey (1998) sugerem o uso de uma distribuição normal inversa neste caso.

Algoritmo 3.4 Amostrar de $\eta \mid \boldsymbol{\beta}, \boldsymbol{D}, \boldsymbol{z}, \boldsymbol{w}, \boldsymbol{\Sigma}$ no MMEPA

1. Inicialize o contador de iterações $t=0$ e especifique um valor inicial para $\eta^{(0)}$;

2. Gere um novo valor para a variável de mistura $\eta^{*}$ através da seguinte distribuição proposta

$$
\eta^{*} \sim \mathcal{N} \mathcal{I}\left(\mu_{E P}^{*}, \sigma_{E P}^{*}\right)
$$

com

$$
\mu_{E P}^{*}=\left[4 c_{0}\left(\boldsymbol{z}_{i}-\boldsymbol{X}_{i} \boldsymbol{\beta}+\boldsymbol{D} \boldsymbol{w}_{i}\right)^{\prime} \boldsymbol{\Sigma}^{-1}\left(\boldsymbol{z}_{i}+\boldsymbol{X}_{i} \boldsymbol{\beta}+\boldsymbol{D} \boldsymbol{w}_{i}\right)\right]^{-1 / 2} \text { e } \sigma_{E P}^{*}=\frac{1}{2},
$$

em que $\mathcal{N} \mathcal{I}(\eta \mid u, v)$ denota uma distribuição normal inversa com função de densidade de probabilidade dada por $\sqrt{\frac{\sigma}{2 \pi \eta_{3}}} \exp \left\{-\frac{\sigma(\eta-\mu)^{2}}{2 \mu^{2} \eta}\right\}$.

3. Gere $u \sim U(0,1)$ e calcule a probabilidade de aceitação $\gamma$ da seguinte forma

$$
\gamma=\min \left\{\frac{h\left(\eta^{*} \mid \alpha, 1\right) / \mathcal{N} \mathcal{I}\left(\eta^{*} \mid 1 / 2,1 / 4\right)}{h(\eta \mid \alpha, 1) / \mathcal{N} \mathcal{I}(\eta \mid 1 / 2,1 / 4)}, 1\right\}
$$

4. Se $u \leq \gamma$ então aceite o novo valor e faça $\eta^{(t+1)}=\eta^{*}$, caso contrário rejeite e faça $\eta^{(t+1)}=\eta$.

5. Incremente o contador de $t$ para $t+1$ e volte ao passo 2 .

Um variável $\eta \sim \mathcal{N} \mathcal{I}(\boldsymbol{\mu}, \boldsymbol{\Sigma})$ pode ser gerada através dos seguimos os passos:

- Passo 1. Gere $Z \sim \mathcal{N}(0,1)$, faça $\xi=Z^{2}$, e calcule $\eta^{*}=\mu+\frac{\mu^{2} \xi}{2 \sigma}-\frac{\mu}{2 \sigma} \sqrt{4 \mu \sigma \xi+\mu^{2} \xi^{2}}$.

- Passo 2. Gere $U \sim U(0,1)$ e faça $\eta=\eta^{*}$ se $U \leq \frac{\mu}{\mu+\eta}$ e $\eta=\frac{\mu^{2}}{\eta^{*}}$ se caso contrário.

Chen e Dey (1998) notou que o cálculo da probabilidade de aceitação dado em (3) para o MMEPA é exatamente o mesmo que foi encontrado para o MMSA. 


\section{Capítulo 4}

\section{Estudos de Simulação}

É conduzido, neste capítulo, dois estudos de simulação para ilustrar o comportamento dos algoritmos de Monte Carlo via Cadeias de Markov (MCMC) apresentados no Capítulo 3. Os estudos consistem em estimar os parâmetros interesse em amostras geradas a partir de modelos multivariados probito assimétricos, com diferentes valores para os parâmetros de forma e tamanhos distintos de amostras. O estudo de simulação considera o caso bivariado.

As amostras foram obtidas através das seguintes etapas:

- Etapa 1: Escolhe-se alguns valores para os vetores de parâmetros fixos $\boldsymbol{\delta}$ e para o vetor de parâmetros de interesse $\boldsymbol{\theta}=(\boldsymbol{\beta}, \sigma)^{\prime}$, e define-se os tamanhos amostrais $n$ e o número de réplicas para cada configuração $R$.

- os valores escolhidos para o vetor de parâmetros de forma são $\boldsymbol{\delta}_{1}=(0,0)^{\prime}, \boldsymbol{\delta}_{2}=(3,3)^{\prime}$, $\boldsymbol{\delta}_{3}=(0,2)^{\prime}, \boldsymbol{\delta}_{4}=(1,3)^{\prime}$ e $\boldsymbol{\delta}_{5}=(1,-1)^{\prime}$. Estes valores foram escolhidos com o objetivo de obter modelos sem assimetria e com assimetria baixa, moderada, alta e contrária.

- os valores escolhidos para os coeficientes da regressão e para o parâmetro de dependência são, respectivamente, $\boldsymbol{\beta}=(16,3,8,4)^{\prime}$ e $\sigma=0,5$, em que $\sigma$ é o parâmetro de correlação da matriz

$$
\boldsymbol{\Sigma}=\left(\begin{array}{ll}
1 & \sigma \\
\sigma & 1
\end{array}\right)
$$

Nesta abordagem temos que $\boldsymbol{\Sigma}$ é a matriz de escala especificada pelo vetor aleatório latente que define a função de ligação bivariada. Estes valores serão chamados no decorrer do estudo de valores reais dos parâmetros.

- os tamanhos amostrais definidos são $n_{1}=100, n_{2}=500$ e $n_{3}=1.000$. O número de réplicas de Monte Carlo foi $R=1.000$. Estes tamanhos amostrais tem por fim verificar se ocorre melhora na estimação dos parâmetros de interesse com o aumento do tamanho da amostra.

- Etapa 2: Gera-se as variáveis explicativas

$$
\boldsymbol{X}_{i}=\left(\begin{array}{cc}
\boldsymbol{x}_{i 1}^{\prime} & \mathbf{0}^{\prime} \\
\mathbf{0}^{\prime} & \boldsymbol{x}_{i 2}^{\prime}
\end{array}\right), i=1, \ldots, n,
$$


em que $\boldsymbol{x}_{i 1}^{\prime}=\left(1, x_{i 12}\right)$ e $\boldsymbol{x}_{i 2}^{\prime}=\left(1, x_{i 22}\right)$, simulando valores das seguintes distribuições uniformes,

$$
x_{i 12} \sim U\left(a_{1}, b_{1}\right) \text { e } x_{i 22} \sim U\left(a_{2}, b_{2}\right),
$$

com $U(a, b)$ denotando a distribuição uniforme contínua no intervalo $(a, b)$. As quantidades $a_{1}, b_{1}, a_{2}$, e $b_{2}$ são dadas por

$$
a_{1}=\frac{\Phi(0,01)-\beta_{12}}{\beta_{12}}, b_{1}=\frac{\Phi(0,99)-\beta_{12}}{\beta_{12}}, a_{2}=\frac{\Phi(0,01)-\beta_{21}}{\beta_{22}} \text { e } b_{2}=\frac{\Phi(0,99)-\beta_{21}}{\beta_{22}},
$$

em que $\Phi(t)$ denota a função de distribuição acumulada da distribuição normal padrão avaliada no ponto $t$. As covariáveis $\boldsymbol{X}_{i}$ foram geradas desta forma para garantir que as probabilidades resultantes dos modelos multivariados probito assimétrico sejam distribuídas em todo o intervalo $[0,1]$. Ou seja, espera-se que as probabilidades apresentem valores próximos de 0 (zero) e de 1 (um).

- Passo 3: Gera-se os vetores de respostas para cada indivíduo, $\boldsymbol{Y}_{i}=\left(Y_{i 1}, Y_{i 2}\right)^{\prime}$, através das variáveis explicativas $\boldsymbol{X}_{i}$ para cada configuração dos parâmetros $\boldsymbol{\beta}, \boldsymbol{\Sigma}$ e $\boldsymbol{\delta}$, utilizando a representação estocástica da distribuição normal assimétrica definida em (2.29),da seguinte forma.

- Gera-se vetores aleatórios latentes $\boldsymbol{\epsilon}_{i}$ e $\boldsymbol{W}_{i}$ das distribuições

$$
\boldsymbol{\epsilon}_{i} \sim \mathcal{N}_{2}(\mathbf{0} ; \boldsymbol{\Sigma}) \text { e } \boldsymbol{W}_{i} \sim \mathcal{N}_{2}^{+}(\mathbf{0} ; \boldsymbol{I})
$$

em que $\mathcal{N}_{2}^{+}(\boldsymbol{\mu} ; \boldsymbol{\Sigma})$ denota a distribuição normal bivariada positiva com vetor de parâmetros de localização $\boldsymbol{\mu}$ e matriz de dispersão $\boldsymbol{\Sigma}$;

- obtem-se o vetor latente $\boldsymbol{Z}_{i}$ fazendo

$$
\boldsymbol{Z}_{i}=\boldsymbol{X}_{i} \boldsymbol{\beta}+\boldsymbol{D} \boldsymbol{W}_{i}+\boldsymbol{\epsilon}_{i}
$$

em que $\boldsymbol{D}=\operatorname{diag}(\boldsymbol{\delta})$

- obtem-se o vetor de respostas $\boldsymbol{Y}_{i}=\left(Y_{i 1}, Y_{i 2}\right)^{\prime}$ através dos valores gerados no passo anterior pelo vetor $\boldsymbol{Z}_{i}$ da seguinte forma:

$$
Y_{i j}= \begin{cases}1 & \text { se } Z_{i j}>0 \\ 0 & \text { se } Z_{i j} \leq 0\end{cases}
$$

para $j=1,2$ e $i=1, \ldots, n$. Cada amostra simulada por este processo é composta por vetores de variáveis respostas $\boldsymbol{Y}_{1}, \boldsymbol{Y}_{2}, \ldots, \boldsymbol{Y}_{n}$ e suas respectivas variáveis explicativas associadas $\boldsymbol{X}_{1}, \boldsymbol{X}_{2}, \ldots, \boldsymbol{X}_{n}$.

Ao fim do processo de simulação são geradas 18.000 amostras distintas, sendo 1.000 réplicas para cada um dos 18 modelos considerados, isto é, para cada combinação das quantidades $\left\{\left(\boldsymbol{\delta}_{l}, n_{t}\right) ; l=\right.$ $1, \ldots, 5, t=1,2,3\}$. Os coeficientes da regressão e o parâmetro de dependência foram considerados, respectivamente, iguais a $\boldsymbol{\beta}=(16,3,8,4)$ e $\sigma=0,5$ em todas as réplicas. 
Na próxima seção utilizamos os algoritmos MCMC apresentados no Capítulo 3 para estimar os parâmetros de interesse nos 18.000 conjuntos de dados gerados no processo de simulação apresentado anteriormente. O estudo de simulação é dividido em duas partes, as quais são discutidas em detalhes a seguir. Todas as simulações são realizadas usando a linguagem de programação matricial ox (Doornik, 2006).

\subsection{Estudo de simulação I}

A primeira parte concentra-se em verificar se, para alguns valores fixos dos parâmetros de forma $\boldsymbol{\delta}$, a amostra simulada da distribuição a posteriori do vetor de parâmetros de interesse $\boldsymbol{\theta}=(\boldsymbol{\beta}, \sigma)^{\prime}$ obtida via o algoritmo de Gibbs apresentado no Capítulo 3, fornece boas estimativas pontuais para o vetor de parâmetros reais $\boldsymbol{\beta}=(16,3,8,4)$ e $\sigma=0,5$. Para cada uma das 18.000 amostras, o algoritmo MCMC utilizado assumiu o vetor de parâmetros $\boldsymbol{\delta}$ conhecido e fixo no verdadeiro valor do parâmetro em que a amostra foi gerada.

As distribuições a priori dos parâmetros de interesse consideradas foram as mesmas apresentadas no Capítulo 3 para os coeficientes da regressão $\boldsymbol{\beta}$ e para a matriz de dispersão $\boldsymbol{\Sigma}$. A distribuição normal multivariada considerada para a distribuição a priori de $\boldsymbol{\beta}$ é dada por

$$
\boldsymbol{\beta} \sim \mathcal{N}_{2}\left(\mathbf{0} ; 1000 \boldsymbol{I}_{2}\right),
$$

e a distribuição a priori de $\boldsymbol{\Sigma}$ é dada em (3.30). A escolha destas distribuições a priori auxiliam na construção do algoritmo de Gibbs.

Para cada uma das 18.000 amostras, o vetor de parâmetros $\delta$ foi considerado conhecido e fixo no verdadeiro valor do parâmetro em que a amostra foi gerada durante o processo de estimação. De cada amostra, foram obtidas cadeias de Markov de tamanho 20.000. Destas, as 10.000 primeiras foram descartadas. Ou seja, o período de aquecimento (Burn In) foi de 10.000 iterações. Para verificar a convergência das cadeias de Markov, algumas cadeias de cada configuração foram selecionadas e seus gráficos de médias ergódicas monitorados. Também foram aplicados os testes de GelmanRubin e de Geweke nas 10.000 iterações após o período de aquecimento. O teste de Gelman-Rubin é baseado em análise de variâncias e estima o fator de redução de escala. Por outro lado, o teste de Geweke divide a cadeia em três partes e faz um teste de igualdade de médias entre as partes extremas através de técnicas de séries temporais. Estes métodos são descritos com detalhes em Paulino, Turkman e Murteira (2003). Os testes indicaram que os valores simulados dos parâmetros utilizando os métodos de simulação apresentados no Capítulo 3 podem ser considerados amostras das distribuições a posteriori dos parâmetros de interesse.

A estimativa pontual considerada para o vetor de parâmetros $\boldsymbol{\theta}=(\boldsymbol{\beta}, \sigma)^{\prime}$ para cada uma das configurações de $\left\{\left(\boldsymbol{\delta}_{l}, n_{t}\right) ; l=1, \ldots, 5, t=1,2,3\right\}$, é dada pela média das esperanças a posteriori estimadas nas 1.000 réplicas. Isto é, a estimativa pontual global para cada configuração é da seguinte 
forma.

$$
\overline{\boldsymbol{\theta}}=\frac{1}{R} \sum_{k=1}^{R} \boldsymbol{\theta}^{(k)},
$$

em que $\boldsymbol{\theta}^{(k)}=\left(\beta_{11}^{(k)}, \beta_{12}^{(k)}, \beta_{21}^{(k)}, \beta_{22}^{(k)}, \sigma^{(k)}\right)^{\prime}, k=1, \ldots, R$, denota o vetor de estimativas de $\mathbb{E}[\boldsymbol{\theta} \mid \boldsymbol{Y}]$ em cada amostra gerada de uma particular configuração de parâmetros de forma e tamanho de amostra.

A Tabela 4.1 apresenta as médias $\overline{\boldsymbol{\theta}}$ para cada configuração de parâmetro de forma e tamanho de amostra. Notamos por esta tabela que as médias a posteriori se aproximam dos valores reais a medida que o tamanho de amostra cresce. As Tabelas 4.2 e 4.3 listam, respectivamente, os viéses relativos $\left(\right.$ Viés $\left._{R}\right)$ e os erros quadráticos médios relativos $\left(\mathrm{EQM}_{R}\right)$ dos parâmetros estimados. Estas medidas são definidas, respectivamente, por

$$
\operatorname{Viés}_{R}(\bar{\theta})=\frac{\bar{\theta}-\theta}{\theta} \quad \text { e } \quad \operatorname{EQM}_{R}(\hat{\theta})=\frac{1}{R} \sum_{k=1}^{R}\left(\frac{\hat{\theta}^{(k)}-\theta}{\theta}\right)^{2} .
$$

Note, pela Tabela 4.2, que os viéses relativos dos coeficientes da regressão $\boldsymbol{\beta}$ diminuem com o aumento do tamanho da amostra. Isto também ocorre na Tabela 4.3 com $\mathrm{EQM}_{R}$. Estas medidas nos auxiliam a visualizar melhor a convergência das médias a posteriori para os verdadeiros valores dos parâmetros. Adicionalmente, as Figuras 4.1, 4.2, 4.3, 4.4 e 4.5 apresentam, respectivamente, para cada parâmetro $\beta_{11}, \beta_{12}, \beta_{21}, \beta_{22}$ e $\sigma$, os gráficos boxplots das 1.000 réplicas para cada combinação das quantidades $\left\{\left(\boldsymbol{\delta}_{l}, n_{t}\right) ; l=1,2, \ldots, 5, t=1,2,3\right\}$. A linha pontilhada denota o valor real do parâmetro. Estes resultados mostram que as variâncias das distribuições a posteriori dos parâmetros de interesse diminuem com o crescimento do tamanho da amostra. Além disto, mostram que as medianas também estão próximas dos valores reais dos parâmetros, convergindo para o valor real com acréscimo do tamanho amostral. Todos os algoritmos MCMC foram escritos usando a linguagem de programação matricial ox (Doornik, 2006). 
Tabela 4.1: Valores médios dos parâmetros estimados para cada configuração de parâmetro de forma e tamanho de amostra.

\begin{tabular}{rrrrrrr}
\hline$\left(\delta_{1}, \delta_{2}\right)$ & $n$ & $\beta_{11}$ & $\beta_{12}$ & $\beta_{21}$ & $\beta_{22}$ & $\sigma$ \\
\hline \multirow{4}{*}{$(0,0)$} & 100 & 17,5185 & 3,2867 & 8,7522 & 4,3744 & 0,6605 \\
& 500 & 16,2460 & 3,0458 & 8,1272 & 4,0651 & 0,5266 \\
& 1.000 & 16,0797 & 3,0147 & 8,0296 & 4,0140 & 0,5164 \\
\hline \multirow{3}{*}{$(3,3)$} & 100 & 19,7219 & 3,6528 & 9,8289 & 4,8040 & 0,3230 \\
& 500 & 16,9278 & 3,1659 & 8,4294 & 4,1899 & 0,6258 \\
& 1.000 & 16,5037 & 3,0898 & 8,2109 & 4,0949 & 0,6341 \\
\hline \multirow{3}{*}{$(0,2)$} & 100 & 18,0858 & 3,3914 & 9,3027 & 4,5911 & 0,6302 \\
& 500 & 16,3136 & 3,0584 & 8,1925 & 4,0898 & 0,5895 \\
& 1.000 & 16,1106 & 3,0209 & 8,0757 & 4,0339 & 0,5443 \\
\hline \multirow{3}{*}{$(1,3)$} & 100 & 18,4643 & 3,4428 & 9,7640 & 4,7919 & 0,5096 \\
& 500 & 16,4757 & 3,0854 & 8,2927 & 4,1345 & 0,6279 \\
& 1.000 & 16,1971 & 3,0349 & 8,1382 & 4,0603 & 0,5828 \\
\hline \multirow{3}{*}{$(1,-1)$} & 100 & 17,9842 & 3,3563 & 9,0082 & 4,5504 & 0,6418 \\
& 500 & 16,3419 & 3,0625 & 8,1698 & 4,0949 & 0,5780 \\
& 1.000 & 16,1296 & 3,0241 & 8,0575 & 4,0342 & 0,5203 \\
\hline
\end{tabular}

* $_{\text {os valores reais do parâmetros são } \boldsymbol{\beta}=(16,3,8,4)^{\prime} \text { e } \sigma=0,5}$

Tabela 4.2: Viéses relativos dos parâmetros estimados para cada configuração de parâmetro de forma e tamanho de amostra.

\begin{tabular}{rrrrrrr}
\hline$\left(\delta_{1}, \delta_{2}\right)$ & $n$ & $\beta_{11}$ & $\beta_{12}$ & $\beta_{21}$ & $\beta_{22}$ & $\sigma$ \\
\hline \multirow{3}{*}{$(0,0)$} & 100 & $9,49 \%$ & $9,56 \%$ & $9,40 \%$ & $9,36 \%$ & $32,10 \%$ \\
& 500 & $1,54 \%$ & $1,53 \%$ & $1,59 \%$ & $1,63 \%$ & $5,33 \%$ \\
& 1.000 & $0,50 \%$ & $0,49 \%$ & $0,37 \%$ & $0,35 \%$ & $3,29 \%$ \\
\hline \multirow{3}{*}{$(3,3)$} & 100 & $23,26 \%$ & $21,76 \%$ & $22,86 \%$ & $20,10 \%$ & $35,40 \%$ \\
& 500 & $5,80 \%$ & $5,53 \%$ & $5,37 \%$ & $4,75 \%$ & $25,17 \%$ \\
& 1.000 & $3,15 \%$ & $3,00 \%$ & $2,64 \%$ & $2,37 \%$ & $26,82 \%$ \\
\hline \multirow{2}{*}{$(0,2)$} & 100 & $13,04 \%$ & $13,05 \%$ & $16,28 \%$ & $14,78 \%$ & $26,05 \%$ \\
& 500 & $1,96 \%$ & $1,95 \%$ & $2,41 \%$ & $2,25 \%$ & $17,91 \%$ \\
& 1.000 & $0,69 \%$ & $0,70 \%$ & $0,95 \%$ & $0,85 \%$ & $8,87 \%$ \\
\hline \multirow{2}{*}{$(1,3)$} & 100 & $15,40 \%$ & $14,76 \%$ & $22,05 \%$ & $19,80 \%$ & $1,93 \%$ \\
& 500 & $2,97 \%$ & $2,85 \%$ & $3,66 \%$ & $3,36 \%$ & $25,59 \%$ \\
& 1.000 & $1,23 \%$ & $1,16 \%$ & $1,73 \%$ & $1,51 \%$ & $16,56 \%$ \\
\hline \multirow{3}{*}{$(1,-1)$} & 100 & $12,40 \%$ & $11,88 \%$ & $12,60 \%$ & $13,76 \%$ & $28,37 \%$ \\
& 500 & $2,14 \%$ & $2,08 \%$ & $2,12 \%$ & $2,37 \%$ & $15,61 \%$ \\
& 1.000 & $0,81 \%$ & $0,81 \%$ & $0,72 \%$ & $0,86 \%$ & $4,06 \%$ \\
\hline
\end{tabular}

${ }^{*}$ os valores reais do parâmetros são $\boldsymbol{\beta}=(16,3,8,4)^{\prime}$ e $\sigma=0,5$ 
Tabela 4.3: Erros quadráticos médios relativos $\left(E Q M_{R}\right)$ dos parâmetros estimados para cada configuração de parâmetro de forma e tamanho de amostra.

\begin{tabular}{rrrrrrr}
\hline$\left(\delta_{1}, \delta_{2}\right)$ & $n$ & $\beta_{11}$ & $\beta_{12}$ & $\beta_{21}$ & $\beta_{22}$ & $\sigma$ \\
\hline \multirow{3}{*}{$(0,0)$} & 100 & 4,8293 & 4,8670 & 5,7205 & 5,6552 & 39,9765 \\
& 500 & 0,5683 & 0,5728 & 0,6144 & 0,6021 & 5,5967 \\
& 1.000 & 0,2611 & 0,2590 & 0,2563 & 0,2520 & 2,5962 \\
\hline \multirow{3}{*}{$(3,3)$} & 100 & 21,5613 & 19,1133 & 21,9081 & 17,5359 & 153,4499 \\
& 500 & 2,3183 & 2,1200 & 2,4833 & 2,0670 & 54,8265 \\
& 1.000 & 0,9719 & 0,8905 & 0,9960 & 0,8397 & 30,3906 \\
\hline \multirow{3}{*}{$(0,2)$} & 100 & 5,9151 & 6,0016 & 12,0251 & 10,0305 & 72,5705 \\
& 500 & 0,6274 & 0,6277 & 1,2376 & 1,0772 & 18,1082 \\
& 1.000 & 0,2686 & 0,2649 & 0,5677 & 0,4840 & 8,1247 \\
\hline \multirow{3}{*}{$(1,3)$} & 100 & 8,3323 & 7,7222 & 21,4821 & 17,3076 & 117,5962 \\
& 500 & 0,8982 & 0,8354 & 2,0842 & 1,7446 & 29,6124 \\
& 1.000 & 0,3503 & 0,3255 & 0,9336 & 0,7951 & 15,8382 \\
\hline \multirow{3}{*}{$(1,-1)$} & 100 & 7,1617 & 6,6377 & 6,2822 & 7,2159 & 64,0304 \\
& 500 & 0,8003 & 0,7479 & 0,7262 & 0,8075 & 14,6338 \\
& 1.000 & 0,3795 & 0,3539 & 0,2944 & 0,3361 & 4,6773 \\
\hline
\end{tabular}

${ }^{*}$ os valores reais do parâmetros são $\boldsymbol{\beta}=(16,3,8,4)^{\prime}$ e $\sigma=0,5$

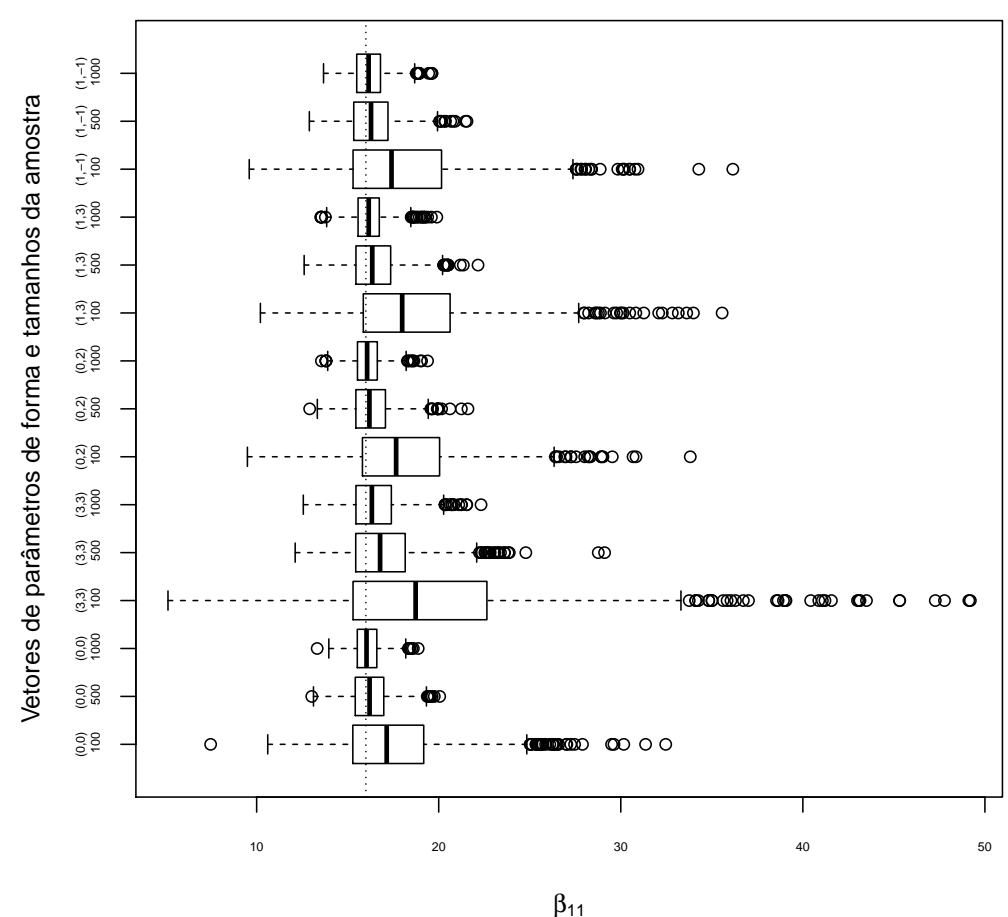

Figura 4.1: Boxplots das distribuições a posteriori de $\beta_{11}$ com $\boldsymbol{\delta}$ e $n$ variando. 


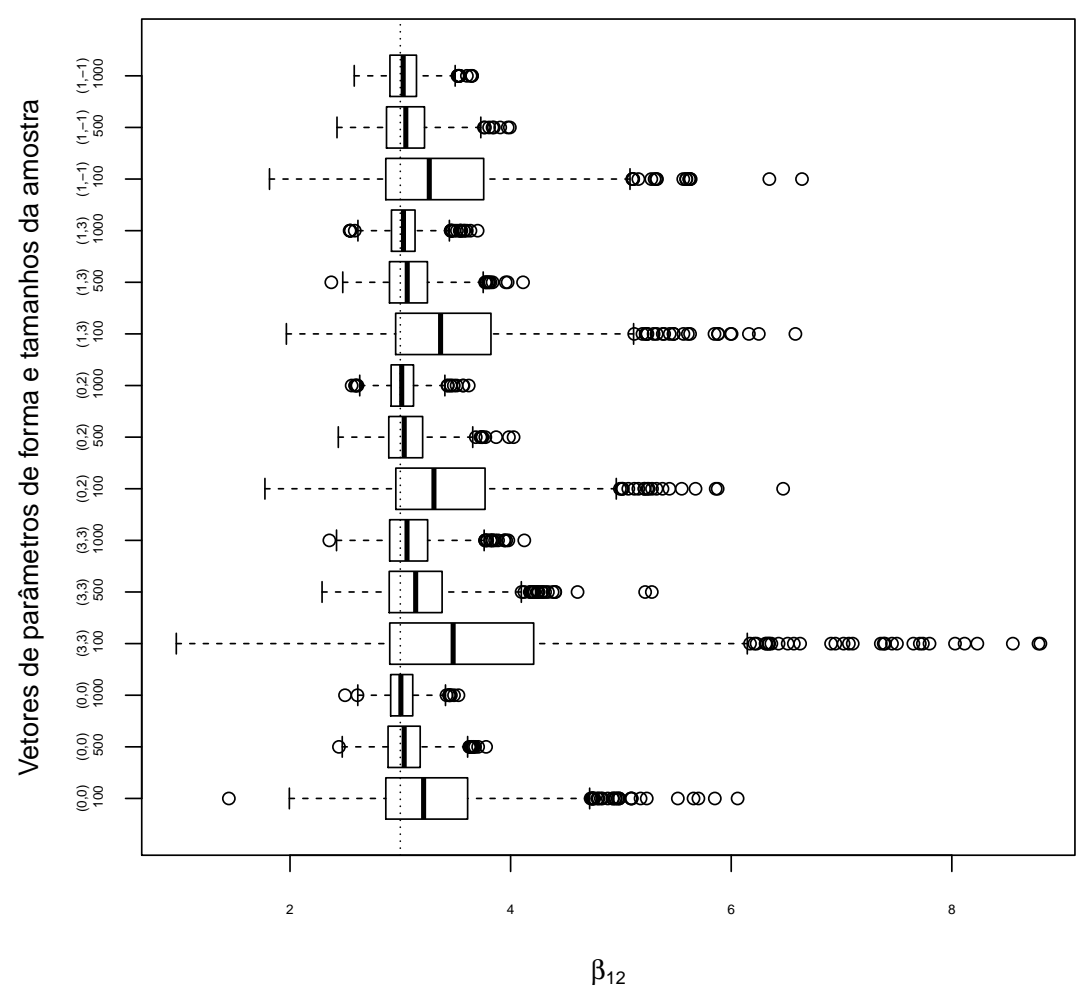

Figura 4.2: Boxplots das distribuições a posteriori de $\beta_{12}$ com $\boldsymbol{\delta}$ e $n$ variando.

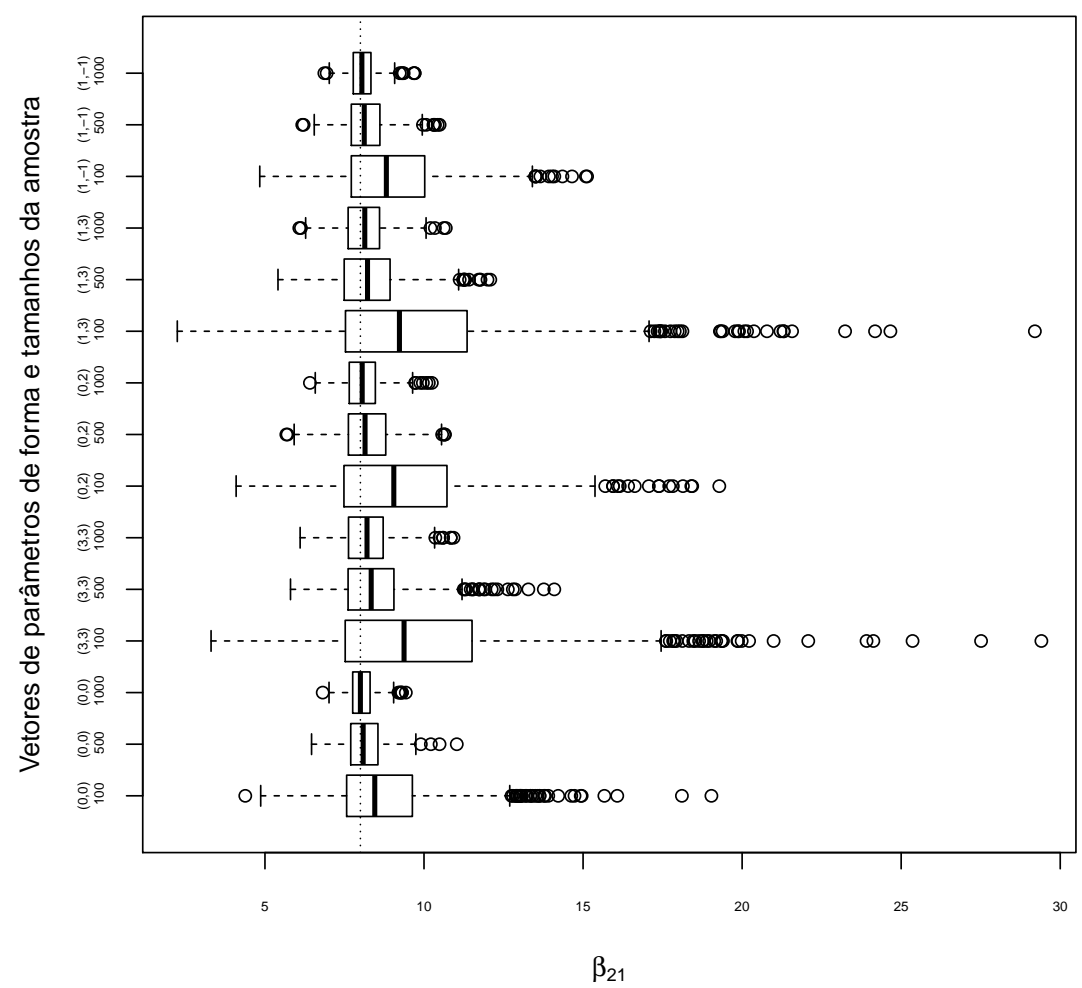

Figura 4.3: Boxplots das distribuições a posteriori de $\beta_{21}$ com $\boldsymbol{\delta}$ e $n$ variando. 


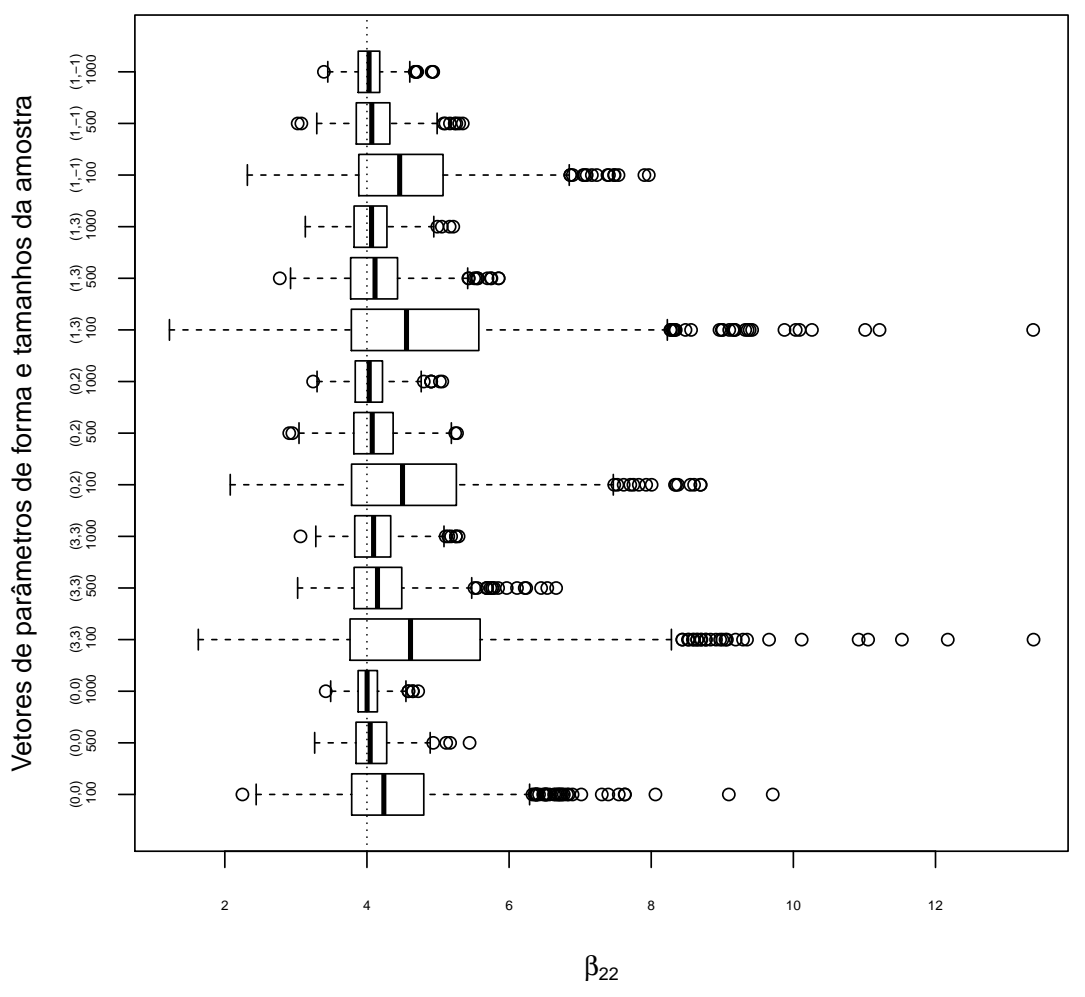

Figura 4.4: Boxplots das distribuições a posteriori de $\beta_{22}$ com $\boldsymbol{\delta}$ e n variando.

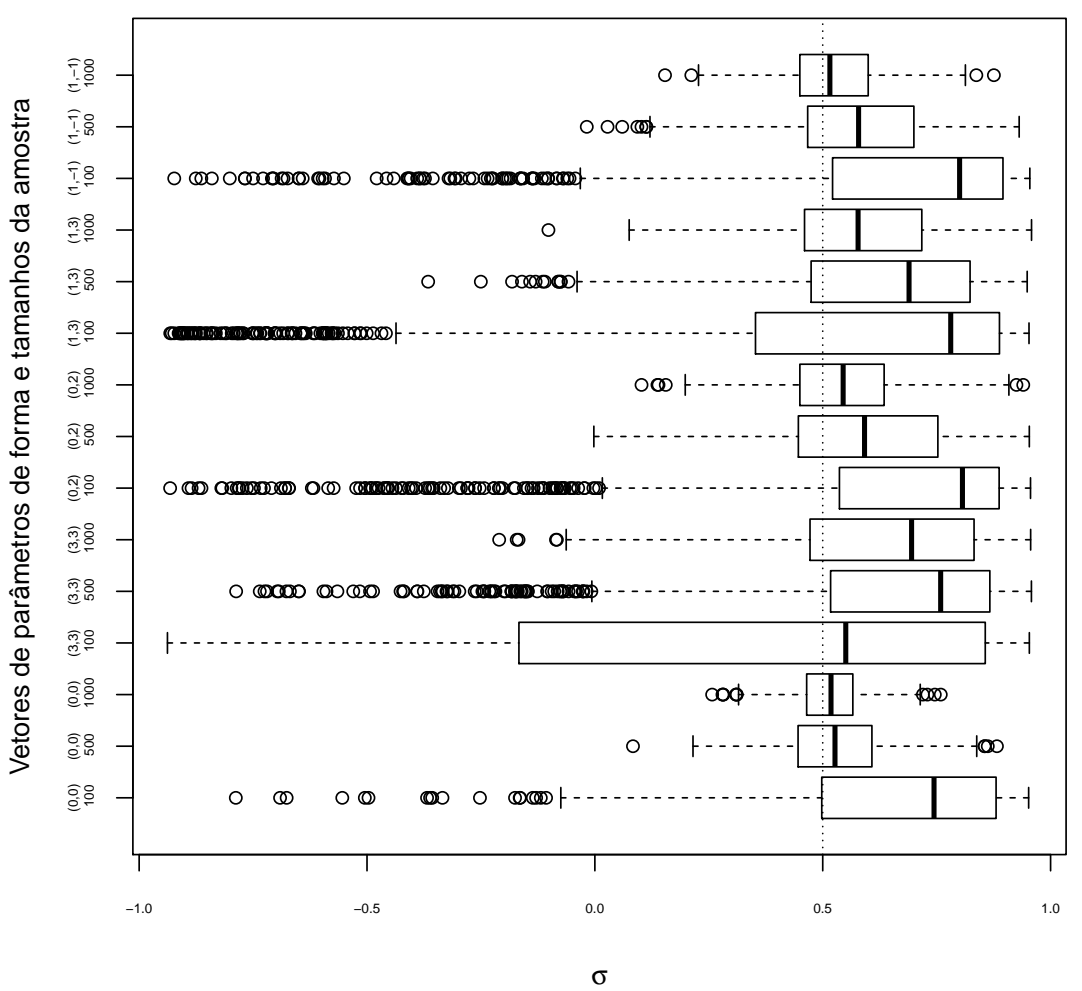

Figura 4.5: Boxplots das distribuições a posteriori de $\sigma_{12}$ com $\boldsymbol{\delta}$ e $n$ variando. 


\subsection{Estudo de simulação II}

O estudo de simulação I ilustrado na seção anterior mostrou que os algoritmos MCMC apresentados no Capítulo 3 são eficientes na estimação dos parâmetros de interesse do modelo multivariado probito assimétrico e que a qualidade das estimativas melhora com o aumento do tamanho amostral. No segundo caso, denominado de estudo de simulação II, o vetor de parâmetros de interesse $\boldsymbol{\theta}=(\boldsymbol{\beta}, \sigma)^{\prime}$ para cada umas das amostras geradas das seguintes configurações do parâmetro de forma $\boldsymbol{\delta}_{2}=(3,3)^{\prime}, \boldsymbol{\delta}_{3}=(0,2)^{\prime}, \boldsymbol{\delta}_{4}=(1,3)^{\prime}$ e $\boldsymbol{\delta}_{5}=(1,-1)^{\prime}$ e tamanhos de amostras $n_{1}=100, n_{2}=500$ e $n_{3}=1.000$, são estimados assumindo, erroneamente, que os conjuntos de dados foram gerados a partir do modelo multivarido probito simétrico, ou seja, é considerado erroneamente $\boldsymbol{\delta}=(0,0)$ no processo de estimação. Esta etapa do estudo tem por objetivo verificar o que ocorre com o ajuste do modelo quando a assimetria da função de ligação é desprezada. A Tabela 4.4 lista as médias das 1.000 réplicas da distribuição a posteriori dos parâmetros de interesse. As duas primeiras colunas da Tabela 4.4 listam, respectivamente, o vetor de parâmetros de forma real utilizado para a geração da amostra e o tamanho da amostra.

Os resultados apresentados na Tabela 4.4 não devem ser comparados com os parâmetros reais $\boldsymbol{\beta}=(16,3,8,4)^{\prime}$ e $\sigma=0,5$, pois a Tabela 4.4 apresenta estimativas pontuais de um modelo diferente daquele que gerou as amostras. No entanto, pode-se usar a soma das diferenças ao quadrado entre as probabilidades ajustadas pelo modelos e a probabilidade real como uma medida de qualidade de ajuste.

$$
\operatorname{EQM}(\hat{p})=\frac{1}{R} \sum_{k=1}^{R}\left[\frac{1}{n} \sum_{i=1}^{n}\left(\widehat{p}_{i}^{(k)}-p_{i}\right)^{2}\right]
$$

com

$$
\hat{p}_{i}{ }^{(k)}=P\left(Y_{i 1}=1, Y_{i 2}=1 \mid x_{i}, \boldsymbol{\beta}^{(k)} ; \rho^{(k)} ; \boldsymbol{\delta}_{C}\right) \quad \text { e } \quad p_{i}=P\left(Y_{i 1}=1, Y_{i 2}=1 \mid x_{i}, \boldsymbol{\beta} ; \rho ; \boldsymbol{\delta}_{R}\right),
$$

em que $\boldsymbol{\beta}=(16,3,8,4)^{\prime}, \sigma=0,5$ e $\boldsymbol{\delta}_{R}$ denotam os conjuntos de valores reais dos parâmetros. $\mathrm{O}$ vetor $\boldsymbol{\delta}_{C}$ representa o vetor de parâmetros de forma considerado no processo de estimação e $\boldsymbol{\beta}^{(k)}$ e $\sigma^{(k)} k=1, \ldots, R$, denotam, respectivamente, as médias das distribuição a posteriori de $\boldsymbol{\beta}$ e $\sigma$ obtidas em cada réplica quando o modelo ajustado.

A Tabela 4.6 lista os erros quadráticos médios das probabilidades obtidas, respectivamente, para os modelos ajustados no estudo de simulação I e no estudo de simulação II. Pode-se notar por esta tabela que o $\operatorname{EQM}(\hat{p})$ diminui quando o tamanho da amostra cresce nos ajuste obtidos no estudo de estimação I. Por outro lado, isto não ocorre no estudo de simulação II, pois o $\operatorname{EQM}(\hat{p})$ permanece constante com a variação do tamanho da amostra. Além disto, o erro quadrático médio obtido pelo processo I é menor que o obtido no estudo de simulação II para qualquer configuração dos parâmetros e tamanho de amostra. Estes resultados nos mostra que o estudo de simulação I, ou seja, fixar o parâmetro de assimetria no valor que gerou a amostra, estima melhor as probabilidades reais. $\mathrm{O}$ erro quadrático médio apresentado na expressão (4.3) nos informam o quão bem ajustadas estão as probabilidades. No entanto, é importante notar que o erro quadrático médio só pode ser calculado quando sabemos quais são os valores reais das probabilidades. Os critérios AIC e o BIC descritos no Apêndice A.1 podem ser utilizadas para comparar os modelos neste estudo de simulação. 
Tabela 4.4: Valores médios dos parâmetros estimados para cada configuração de parâmetro de forma e tamanho de amostra, considerado $\boldsymbol{\delta}=(0,0)$ no processo de estimação.

\begin{tabular}{ccccccc}
\hline$\left(\delta_{1}, \delta_{2}\right)$ & $n$ & $\beta_{11}$ & $\beta_{12}$ & $\beta_{21}$ & $\beta_{22}$ & $\sigma$ \\
\hline \multirow{3}{*}{$(3,3)$} & 100 & 13,2031 & 2,2057 & 7,3020 & 2,9343 & 0,1825 \\
& 500 & 11,9167 & 1,9858 & 6,5902 & 2,6322 & 0,1908 \\
& 1.000 & 11,7782 & 1,9615 & 6,5461 & 2,6134 & 0,1828 \\
\hline \multirow{3}{*}{$(0,2)$} & 100 & 17,5891 & 3,2994 & 7,6622 & 3,2422 & 0,4440 \\
& 500 & 16,2144 & 3,0407 & 6,9941 & 2,9517 & 0,3663 \\
& 1.000 & 16,0603 & 3,0123 & 6,9970 & 2,9535 & 0,3615 \\
\hline \multirow{3}{*}{$(1,3)$} & 100 & 16,2756 & 2,9089 & 7,3092 & 2,9415 & 0,3387 \\
& 500 & 14,8909 & 2,6609 & 6,5572 & 2,6173 & 0,2732 \\
& 1.000 & 14,7375 & 2,6331 & 6,5435 & 2,6109 & 0,2705 \\
\hline \multirow{3}{*}{$(1,-1)$} & 100 & 16,0964 & 2,8748 & 7,0056 & 3,8861 & 0,4957 \\
& 500 & 14,9191 & 2,6668 & 6,4272 & 3,5649 & 0,4010 \\
& 1.000 & 14,7688 & 2,6394 & 6,3323 & 3,5150 & 0,3787 \\
\hline
\end{tabular}

Analisando Tabela 4.6, pode-se notar que os critérios AIC e BIC obtidos no estudo simulação I são menores que as obtidas no estudo de simulação II em todos os casos. Este resultado mostra que estes critérios podem ser utilizados na seleção de modelos multivariados probito assimétrico. A Tabela 4.5 listam os erros qudráticos médios e os critérios AIC e BIC para o modelo multivariado probito simétrico.

Tabela 4.5: Erro quadrático médio, AIC e BIC para o modelo multivariado probito simétrico.

\begin{tabular}{cccc}
\hline $\mathrm{n}$ & $\mathrm{EQM}(p)$ & $\mathrm{AIC}$ & $\mathrm{BIC}$ \\
\hline 100 & 0,0012 & 1799,86 & 1822,89 \\
500 & 0,0002 & 5125,36 & 5156,43 \\
1.000 & 0,0002 & 5740,30 & 5774,85 \\
\hline
\end{tabular}

Concluímos nestes estudos que os algoritmos apresentados no Capítulo 3 fornecem bons resultados na estimação dos parâmetros de interesse, com os resultados melhorando a medida que o tamanho da amostra cresce. Além disto, notamos que a escolha da função de ligação em modelos multivariados binários é importante e, caso seja mal especificada, pode conduzir a modelos pobremente ajustados. Por fim, observamos que as critérios de comparação AIC e BIC podem ser utilizadas para selecionar modelos. 
Tabela 4.6: Erro quadrático médio, AIC e BIC para cada configuração de parâmetro de forma e tamanho de amostra para os estudos de simulação I e II

\begin{tabular}{|c|c|c|c|c|c|c|c|}
\hline \multirow[b]{2}{*}{$\left(\delta_{1}, \delta_{2}\right)$} & \multirow[b]{2}{*}{$n$} & \multicolumn{3}{|c|}{ Estudo de simulação I } & \multicolumn{3}{|c|}{ Estudo de simulação II } \\
\hline & & $\operatorname{EQM}(p)$ & $\mathrm{AIC}$ & BIC & $\operatorname{EQM}(p)$ & AIC & $\mathrm{BIC}$ \\
\hline \multirow{3}{*}{$(3,3)$} & 100 & 0,0184 & 463,89 & 476,92 & 0,3209 & 1460,32 & 1473,34 \\
\hline & 500 & 0,0021 & 2042,83 & 2063,90 & 0,3112 & 4879,80 & 4920,87 \\
\hline & 1.000 & 0,0009 & 4157,91 & 4182,45 & 0,3079 & 9532,33 & 9556,87 \\
\hline \multirow{3}{*}{$(0,2)$} & 100 & 0,0028 & 1013,35 & 1026,37 & 0,0414 & 1427,33 & 1440,36 \\
\hline & 500 & 0,0004 & 4048,07 & 4069,14 & 0,0377 & 4758,54 & 4779,61 \\
\hline & 1.000 & 0,0002 & 7684,62 & 7719,16 & 0,0368 & 9726,61 & 9751,15 \\
\hline \multirow{3}{*}{$(1,3)$} & 100 & 0,0089 & 672,83 & 685,86 & 0,1328 & 1299,97 & 1313,00 \\
\hline & 500 & 0,0011 & 2926,98 & 2948,06 & 0,1307 & 4601,97 & 4623,05 \\
\hline & 1.000 & 0,0005 & 5852,40 & 5876,93 & 0,1301 & 9113,45 & 9137,99 \\
\hline \multirow[b]{2}{*}{$(1,-1)$} & 100 & 0,0040 & 1068,47 & 1081,50 & 0,0451 & 1682,86 & 1695,88 \\
\hline & 500 & 0,0006 & 3948,97 & 3970,04 & 0,0415 & 5111,89 & 5132,96 \\
\hline
\end{tabular}




\section{Capítulo 5}

\section{Aplicação}

Greene (1997) e Chib e Greenberg (1998) analisaram um conjunto de dados referente a uma pesquisa sobre o comportamento dos votos de residentes da cidade de Troy, no estado de Michigan, Estados Unidos da América. Neste estudo 95 moradores foram questionados a responder duas perguntas: a primeira, se tem ou não, pelo menos um filho na escola pública $\left(Y_{1}\right)$; e a segunda, se vota a favor ou contra a criação de um fundo orçamentário para a escola $\left(Y_{2}\right)$. O objetivo desta pesquisa foi estudar o comportamento entre as duas variáveis respostas binárias e algumas variáveis explicativas.

Ambos os trabalhos consideraram as seguintes variáveis explicativas: renda familiar anual (INC); logaritmo natural do valor pago por ano em impostos sobre imóveis por cada família (TAX); número de anos que a família reside na cidade de Troy (YRS), e propuseram o seguinte modelo bivariado probito para o ajuste,

$$
P\left(Y_{i 1}=y_{i 1}, Y_{i 2}=y_{i 2} \mid \boldsymbol{X}_{i}, \boldsymbol{\beta}, \boldsymbol{\Sigma}\right)=\int_{B_{i 2}} \int_{B_{i 1}} \phi_{2}\left(\boldsymbol{z} \mid \boldsymbol{X}_{i} \boldsymbol{\beta}, \boldsymbol{\Sigma}\right) d \boldsymbol{z}, i=1, \ldots, n,
$$

com

$$
B_{i j}= \begin{cases}(0,+\infty) & \text { se } y_{i j}=1 \\ (-\infty, 0] & \text { se } y_{i j}=0\end{cases}
$$

em que $y_{i 1}$ e $y_{i 2}$ denotam, respectivamente, as respostas observadas do $i$-ésimo indivíduo para as questões $Y_{1}$ e $Y_{2}$,

$$
\boldsymbol{X}_{i}=\left(\begin{array}{cc}
\boldsymbol{x}_{i 1} & \mathbf{0}^{\prime} \\
\mathbf{0}^{\prime} & \boldsymbol{x}_{i 2}
\end{array}\right)=\left(\begin{array}{ccccccc}
1 & \mathrm{INC}_{i} & \mathrm{TAX}_{i} & 0 & 0 & 0 & 0 \\
0 & 0 & 0 & 1 & \mathrm{INC}_{i} & \mathrm{TAX}_{i} & \mathrm{YRS}_{i}
\end{array}\right)
$$

representa a matriz de variáveis explicativas associada ao $i$-ésimo indivíduo e

$$
\boldsymbol{\Sigma}=\left(\begin{array}{cc}
1 & \sigma_{12} \\
\sigma_{12} & 1
\end{array}\right)
$$

denota a matriz de dispersão do vetor de variáveis latentes, $\boldsymbol{Z}$, que induziu a função de ligação. No modelo probito (simétrico) o vetor de variáveis latentes é normalmente distribuído com matriz de 
dispersão igual à matriz de correlações $\left(\boldsymbol{Z}_{i} \sim \mathcal{N}_{J}\left(\boldsymbol{X}_{i} \boldsymbol{\beta}, \boldsymbol{\Sigma}\right)\right)$. O vetor de coeficientes da regressão é denotado por $\boldsymbol{\beta}=\left(\boldsymbol{\beta}_{1}^{\prime}, \boldsymbol{\beta}_{2}^{\prime}\right)^{\prime}$, em que $\boldsymbol{\beta}_{1}=\left(\beta_{11}, \beta_{12}, \beta_{13}\right)^{\prime}$ representa o vetor de coeficientes associados à variável resposta $Y_{1}$ e $\boldsymbol{\beta}_{2}=\left(\beta_{21}, \beta_{22}, \beta_{23}, \beta_{24}\right)^{\prime}$ representa o vetor de coeficientes associados à variável resposta $Y_{2}$.

A Tabela 5.1 apresenta algumas estatísticas a posteriori para o modelo bivariado probito definido em (5.1), considerando a priori distribuições normais independentes com médias zero e variâncias 1.000 para cada um dos coeficientes da regressão e a distribuição dada em (3.30) para a matriz de dispersão. Nós utilizamos o algoritmo de Gibbs apresentando no Capítulo 3 para a obtenção das amostras a posteriori dos parâmetros de interesse. No processo de simulação, as primeiras 10.000 iterações foram consideradas como iterações do período de aquecimento (Burn In) e descartadas da análise. Nós saltamos de 10 em 10 iterações após o período de aquecimento para obter cadeias menos correlacionadas. Todos os algoritmos MCMC foram escritos usando a linguagem de programação matricial Ox (Doornik, 2006). A convergência das cadeias geradas foram verificadas utilizando diversos procedimentos de diagnósticos incluídos no pacote CODA, por exemplo, o teste de Geweke e os gráficos do traço e das médias ergódicas (ver, Plummer et al., 2006). Ao final do processo a convergência foi alcançada e as inferências foram baseadas em amostras de tamanho 2.000 para cada parâmetro. A Figura 5.1 nos permite visualizar o comportamento destas cadeias para cada um dos parâmetros de interesse.

Tabela 5.1: Estatísticas a posteriori para o modelo bivariado probito ajustado ao conjunto de dados sobre o comportamento de votos na cidade de Troy.

\begin{tabular}{rrrrrrrrr}
\hline & & & & \multicolumn{2}{c}{ HPD de $90 \%$} & \multicolumn{2}{c}{ HPD de $95 \%$} \\
\multicolumn{2}{r}{ Parâmetro } & Média & D. P. & Mediana & Inferior & Superior & Inferior & Superior \\
\hline $\boldsymbol{\beta}_{1}$ & $\beta_{11}$ & $-4,7745$ & 3,8955 & $-4,74775$ & $-11,0486$ & 1,4712 & $-12,7536$ & 2,4472 \\
& $\beta_{12}$ & 0,1109 & 0,4425 & 0,10858 & $-0,6174$ & 0,8016 & $-0,7643$ & 0,9463 \\
& $\beta_{13}$ & 0,6790 & 0,5613 & 0,67657 & $-0,1600$ & 1,6537 & $-0,3976$ & 1,7877 \\
$\boldsymbol{\beta}_{2}$ & $\beta_{21}$ & $-0,3296$ & 3,9753 & $-0,32010$ & $-6,9984$ & 5,9458 & $-7,9692$ & 7,2561 \\
& $\beta_{22}$ & 1,0451 & 0,4470 & 1,02217 & 0,2865 & 1,7398 & 0,1809 & 1,9397 \\
& $\beta_{23}$ & $-1,3839$ & 0,5865 & $-1,38767$ & $-2,3268$ & $-0,4191$ & $-2,4757$ & $-0,2035$ \\
& $\beta_{24}$ & $-0,0176$ & 0,0144 & $-0,01727$ & $-0,0399$ & 0,0068 & $-0,0440$ & 0,0126 \\
$\sigma_{12}$ & & 0,3683 & 0,2066 & 0,37830 & 0,0392 & 0,7223 & $-0,0131$ & 0,7719 \\
\hline
\end{tabular}

Analisando a Tabela 5.1 observamos que as médias e medianas a posteriori estão próximas, indicando que as distribuições marginais a posteriori dos parâmetros de interesse são simétricas. Notamos também que somente os coeficientes da regressão $\beta_{22}$ e $\beta_{23}$ e o parâmetro de dependência $\sigma_{12}$ não incluem o zero em seus respectivos intervalos HPD de $90 \%$ de credibilidade. O mesmo não ocorre quando a credibilidade do intervalo é aumentada para $95 \%$, neste caso, apenas os coeficientes da regressão $\beta_{22}$ e $\beta_{23}$ não incluem o zero em seus intervalos. Estes resultados poderiam nos levar a discutir se os coeficientes da regressão cujos seus respectivos intervalos HPD incluem o zero devem ou não serem considerados nulos. No entanto, assim como nas análises empreendidas por Greene (1997) e Chib e Greenberg (1998), está discusão não faz parte do escopo deste trabalho.

No caso do parâmetro de dependência, é importante notar que o parâmetro $\sigma_{12}$ não representa 


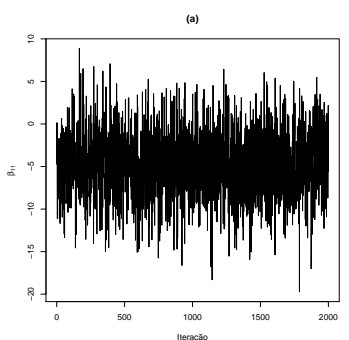

(a)

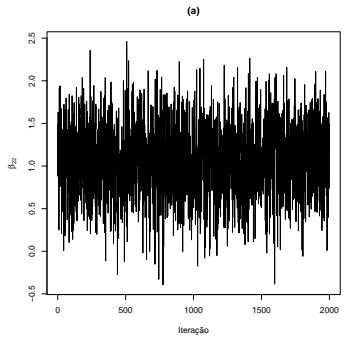

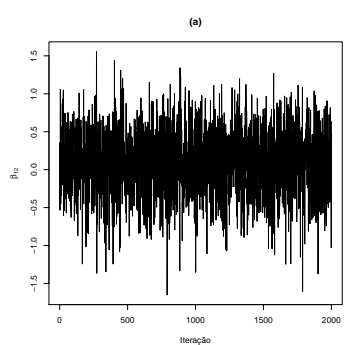

(a)

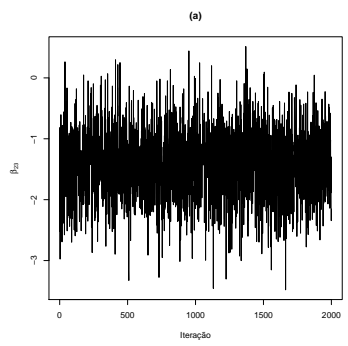

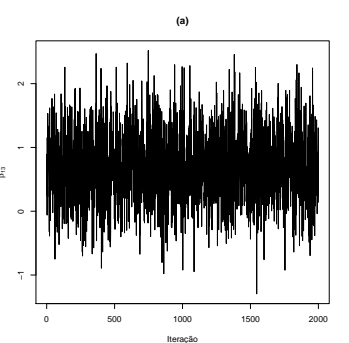
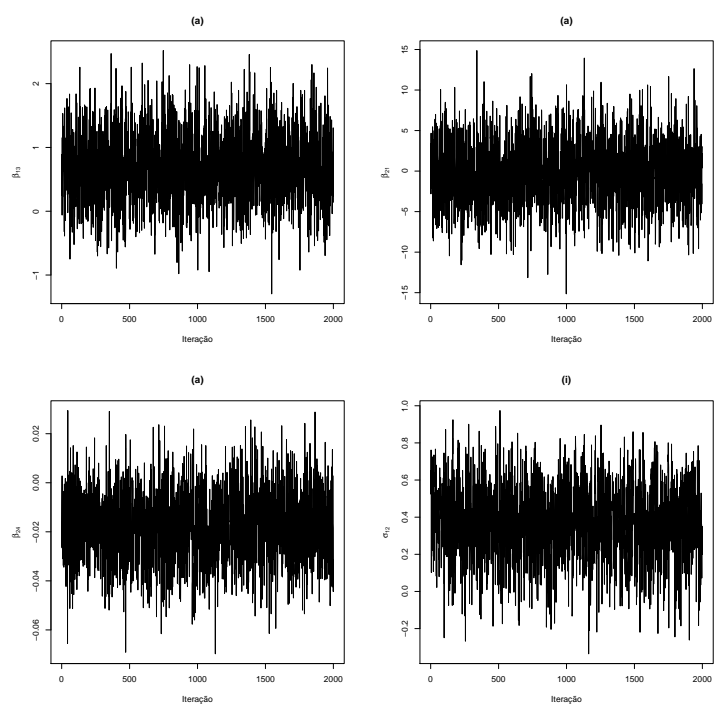

Figura 5.1: Cadeias de Markov das distribuições a posteriori dos parâmetros de interesse.

a correlação entre as variáveis $Y_{i 1}$ e $Y_{i 2}$, a qual é definida no modelo multivariado binário como

$$
\rho\left(Y_{i 1}, Y_{i 2}\right)=\frac{\mathbb{E}\left(Y_{i 1} Y_{i 2} \mid \boldsymbol{X}_{i}, \boldsymbol{\beta}, \boldsymbol{\Sigma}\right)-\mathbb{E}\left(Y_{i 1} \mid \boldsymbol{X}_{i}, \boldsymbol{\beta}, \boldsymbol{\Sigma}\right) \mathbb{E}\left(Y_{i 2} \mid \boldsymbol{X}_{i}, \boldsymbol{\beta}, \boldsymbol{\Sigma}\right)}{\sqrt{\operatorname{Var}\left(Y_{i 1} \mid \boldsymbol{X}_{i}, \boldsymbol{\beta}, \boldsymbol{\Sigma}\right) \operatorname{Var}\left(Y_{i 2} \mid \boldsymbol{X}_{i}, \boldsymbol{\beta}, \boldsymbol{\Sigma}\right)}} .
$$

em que

$$
\begin{aligned}
\mathbb{E}\left(Y_{i 1} Y_{i 2} \mid \boldsymbol{X}_{i}, \boldsymbol{\beta}, \boldsymbol{\Sigma}\right) & =P\left(Y_{i 1}=1, Y_{i 2}=1 \mid \boldsymbol{X}_{i}, \boldsymbol{\beta}, \boldsymbol{\Sigma}\right) \\
\mathbb{E}\left(Y_{i j} \mid \boldsymbol{X}_{i}, \boldsymbol{\beta}, \boldsymbol{\Sigma}\right) & =P\left(Y_{i j}=1 \mid \boldsymbol{x}_{i}, \boldsymbol{\beta}_{j}\right), j=1,2 \\
\operatorname{Var}\left(Y_{i j} \mid \boldsymbol{X}_{i}, \boldsymbol{\beta}, \boldsymbol{\Sigma}\right) & =P\left(Y_{i j}=1 \mid \boldsymbol{x}_{i}, \boldsymbol{\beta}_{j}\right)\left[1-P\left(Y_{i j}=1 \mid \boldsymbol{x}_{i}, \boldsymbol{\beta}_{j}\right)\right], j=1,2 .
\end{aligned}
$$

Pode-se observar na expressão (5.2) que a correlação entre as componentes do vetor $\boldsymbol{Y}_{i}=$ $\left(Y_{i 1}, Y_{i 2}\right)^{\prime}$ pode ser diferente da correlação entre as componentes do vetor $\boldsymbol{Y}_{i^{\prime}}=\left(Y_{i^{\prime} 1}, Y_{i^{\prime} 2}\right)^{\prime}$.

Temos, da expressão (5.2), que a correlação entre as variáveis $Y_{i 1}$ e $Y_{i 2}$ no modelo bivariado probito definido em (5.1) é dada por

$$
\rho\left(Y_{i 1}, Y_{i 2} \mid \boldsymbol{X}_{i}, \boldsymbol{\beta}, \boldsymbol{\Sigma}\right)=\frac{\Phi_{2}\left(\boldsymbol{X}_{i} \boldsymbol{\beta} \mid \mathbf{0}, \boldsymbol{\Sigma}\right)-\Phi\left(\boldsymbol{x}_{i 1} \boldsymbol{\beta}_{1}\right) \Phi\left(\boldsymbol{x}_{i 2} \boldsymbol{\beta}_{2}\right)}{\sqrt{\Phi\left(\boldsymbol{x}_{i 1} \boldsymbol{\beta}_{1}\right)\left[1-\Phi\left(\boldsymbol{x}_{i 1} \boldsymbol{\beta}_{1}\right)\right] \Phi\left(\boldsymbol{x}_{i 2} \boldsymbol{\beta}_{2}\right)\left[1-\Phi\left(\boldsymbol{x}_{i 2} \boldsymbol{\beta}_{2}\right)\right]}},
$$

em que $\Phi(t)$ denota a função de distribuição acumulada (fdp) da distribuição normal padrão univariada avaliada em $t$ e

$$
\Phi_{2}(\boldsymbol{t} \mid \mathbf{0}, \boldsymbol{\Sigma})=\int_{-\infty}^{t_{2}} \int_{-\infty}^{t_{2}} \phi_{2}(\boldsymbol{s} \mid \mathbf{0}, \boldsymbol{\Sigma}) d \boldsymbol{s}
$$

representa a fdp da distribuição normal bivariada com vetor de médias $\mathbf{0}$ e matriz de variânciascovariâncias $\boldsymbol{\Sigma}$ avaliada no vetor $\boldsymbol{t}=\left(t_{1}, t_{2}\right)$. Da expressão (5.3) podemos observar que as correlações $\rho\left(Y_{i 1}, Y_{i 2}\right)$ e $\rho\left(Y_{i^{\prime} 1}, Y_{i^{\prime} 2}\right)$ serão iguais somente no caso $\boldsymbol{X}_{i} \boldsymbol{\beta}=\boldsymbol{X}_{i^{\prime}} \boldsymbol{\beta}$. Este resultado pode ser estendido para todos os modelos com funções de ligação elíptica assimétrica.

A Figura 5.2 (a) apresenta as estimativas pontuais das correlações $\rho\left(Y_{i 1}, Y_{i 2}\right)$ para cada indivíduo pertencente ao conjunto de dados. A linha tracejada no ponto 0,1910 indica a média das 
(a)

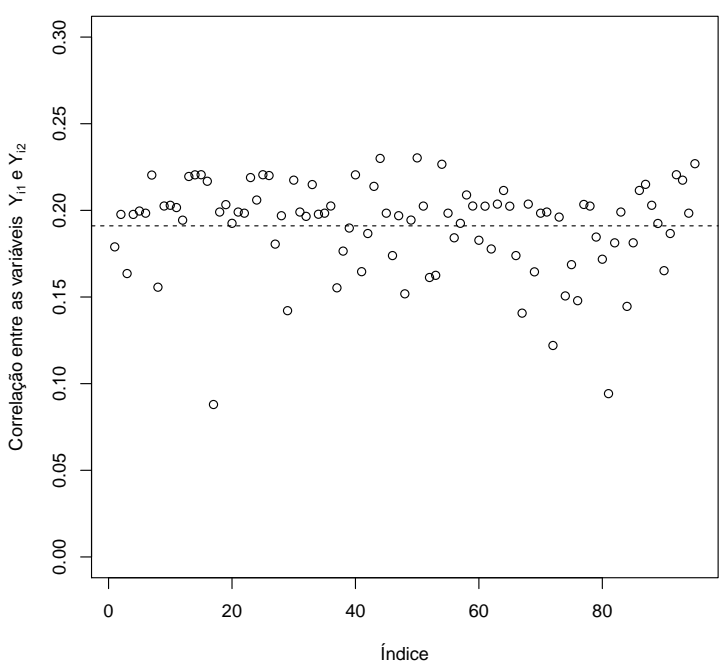

(b)

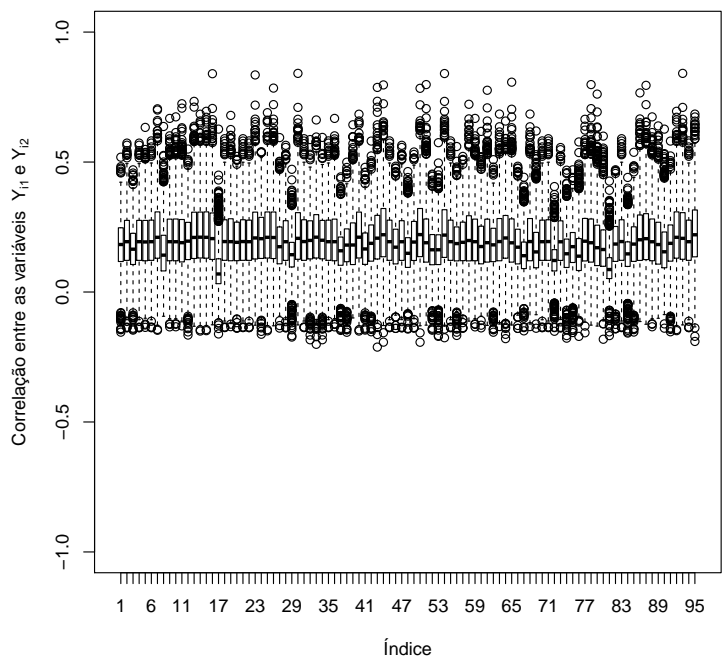

Figura 5.2: Estimativas pontuais das correlações (a) e gráficos boxplot das distribuições a posteriori das correlações (b).

correlações. A Figura 5.2 (b) apresenta uma análise mais rica das correlações de cada indivíduos, apresentando os gráficos boxplots das distribuições a posteriori das correlações para cada indivíduo. A correlação média é 0,1910 e o desvio-padrão médio é 0,1139. Estes resultados indicam que existe uma correlação de aproximadamente 0,2 entre as variáveis $Y_{1}$ e $Y_{2}, i=1, \ldots, n$.

Os resultados do modelo bivariado probito ajustado nesta aplicação é similar aos resultados obtidos por Greene (1997) e Chib e Greenberg (1998). No entanto, diferentemente do que apresentamos aqui, ambos os trabalhos anteriormente citados discutiram a correlação entre as variáveis $Y_{1}$ e $Y_{2}$ somente no nível da variável latente que induziu a estrutura de dependência. Como o modelo probito simétrico é um caso particular do modelo probito assimétrico, consideraremos na próxima seção a possibilidade do modelo bivariado probito assimétrico fornecer um melhor ajuste ao conjunto dados que o modelo bivariado probito.

\subsection{Comparação de modelos}

Na presente seção aplicamos as propostas de comparação de modelos discutidas no Apêndice A.1 em 40 (quarenta) modelos concorrentes ajustados ao conjunto de dados ilustrado no seção anterior. Todos estes modelos concorrentes pertencem à classe de modelos multivariados probito assimétrico.

Seja $\mathcal{M}_{1}, \mathcal{M}_{2}, \ldots, \mathcal{M}_{40}$ modelos concorrentes pertencentes à classe de modelos multivariados probito assimétrico e considere que a função de verossimilhança para o modelo $\mathcal{M}_{k}$, denotada por $L\left(\boldsymbol{\beta}, \boldsymbol{\Sigma} ; \boldsymbol{y}, \mathcal{M}_{k}\right)$, é definida da seguinte forma.

$$
L\left(\boldsymbol{\beta}, \boldsymbol{\Sigma} ; \boldsymbol{y}, \mathcal{M}_{k}\right)=\prod_{i=1}^{n} P\left(\boldsymbol{Y}_{i}=\boldsymbol{y}_{i} \mid \boldsymbol{\beta}, \boldsymbol{\Gamma}, \mathcal{M}_{k}\right)
$$


em que

$$
P\left(\boldsymbol{Y}_{i}=\boldsymbol{y}_{i} \mid \boldsymbol{\beta}, \boldsymbol{\Sigma}, \mathcal{M}_{k}\right)=\int_{B_{i 2}} \int_{B_{i 1}}\left[\int_{0}^{\infty} \int_{0}^{\infty} \phi_{2}\left(\boldsymbol{z} \mid \boldsymbol{x}_{i} \boldsymbol{\beta}+\boldsymbol{D}_{k} \boldsymbol{w}, \boldsymbol{\Sigma}\right) \phi_{2}(\boldsymbol{w} \mid \mathbf{0}, \boldsymbol{I}) d \boldsymbol{w}\right] d \boldsymbol{z}
$$

$\operatorname{com} \boldsymbol{D}_{k}=\operatorname{diag}\left(\delta_{k 1}, \delta_{k 2}\right)$ denotando uma matriz diagonal $2 \times 2$ conhecida e fixa associada ao modelo $\mathcal{M}_{k}$. A escolha do vetor $\boldsymbol{\delta}_{k}=\left(\delta_{k 1}, \delta_{k 2}\right)^{\prime}$ define o grau de assimetria considerado para o modelo $\mathcal{M}_{k}$. Nós consideramos neste estudo que o modelo $\mathcal{M}_{1}$ está associado ao vetor $\boldsymbol{\delta}_{1}=(0,0)^{\prime}$, ou seja, o modelo $\mathcal{M}_{1}$ representa o modelo probito definido na seção anterior. A Tabela 5.2 apresenta os vetores de forma $\boldsymbol{\delta}$ para todos os 40 modelos concorrentes .

Tabela 5.2: Configurações do vetores $\boldsymbol{\delta}_{k}=\left(\delta_{k 1}, \delta_{k 2}\right)^{\prime}$ para cada um modelos concorrentes $\mathcal{M}_{k}, k=1, \ldots, 40$.

\begin{tabular}{|r|rr|r|rr|r|rr|r|rr|}
\hline$k$ & $\delta_{k 1}$ & $\delta_{k 2}$ & $k$ & $\delta_{k 1}$ & $\delta_{k 2}$ & $k$ & $\delta_{k 1}$ & $\delta_{k 2}$ & $k$ & $\delta_{1}$ & $\delta_{2}$ \\
\hline 1 & 0,00 & 0,00 & 11 & 0,20 & 0,00 & 21 & 0,00 & $-0,30$ & 31 & 0,50 & 0,00 \\
2 & 0,10 & 0,00 & 12 & 0,00 & $-0,20$ & 22 & $-0,30$ & 0,00 & 32 & 0,00 & 0,50 \\
3 & 0,00 & 0,10 & 13 & 0,00 & 0,20 & 23 & $-0,30$ & $-0,30$ & 33 & 0,00 & $-0,50$ \\
4 & $-0,10$ & 0,00 & 14 & 0,10 & $-0,20$ & 24 & 0,30 & 0,30 & 34 & $-0,50$ & 0,00 \\
5 & 0,00 & $-0,10$ & 15 & 0,20 & $-0,10$ & 25 & 0,30 & $-0,30$ & 35 & $-0,50$ & $-0,50$ \\
6 & $-0,10$ & $-0,10$ & 16 & $-0,10$ & 0,20 & 26 & $-0,30$ & 0,30 & 36 & 0,50 & 0,50 \\
7 & 0,10 & 0,10 & 17 & $-0,20$ & 0,10 & 27 & 0,20 & $-0,20$ & 37 & 0,00 & 1,00 \\
8 & 0,10 & $-0,10$ & 18 & $-0,20$ & $-0,20$ & 28 & $-0,20$ & 0,20 & 38 & 1,00 & 0,00 \\
9 & $-0,10$ & 0,10 & 19 & 0,20 & 0,20 & 29 & 0,30 & 0,00 & 39 & 1,00 & 1,00 \\
10 & $-0,20$ & 0,00 & 20 & 0,25 & 0,25 & 30 & 0,00 & 0,30 & 40 & $-1,00$ & $-1,00$ \\
\hline
\end{tabular}

Nós consideramos aqui que as matrizes de variáveis explicativas $\boldsymbol{X}_{i}$, o vetor de coeficientes $\boldsymbol{\beta}$ e a matriz de dispersão $\boldsymbol{\Sigma}$, para o modelo $\mathcal{M}_{k}$, são as mesmas definidas anteriormente para o modelo bivariado probito apresentado na expressão (5.1). No entanto, a matriz de escala $\boldsymbol{\Sigma}$ somente será igual à matriz de variâncias-covariâncias da distribuição da variável latente que induziu a função de ligação no modelo bivariado probito. A matriz de variâncias-covariâncias da distribuição que induz a função de ligação probito assimétrica é dada por

$$
\boldsymbol{\Gamma}=\boldsymbol{\Sigma}+\boldsymbol{D}_{k}^{2}=\left(\begin{array}{cc}
1+\delta_{k 1}^{2} & \sigma_{12} \\
\sigma_{12} & 1+\delta_{k 2}^{2}
\end{array}\right)
$$

Como foi dito anteriormente, observe que a matriz $\boldsymbol{\Gamma}$ será igual a matriz $\boldsymbol{\Sigma}$ somente no caso em que todos os parâmetros de forma são nulos. Sahu, Dey e Branco (2003) argumentam que, dado que $\boldsymbol{D}_{k}$ é uma matriz diagonal, a introdução de assimetria no modelo não afeta a estrutura de correlação. O vetor de variáveis latente $\boldsymbol{Z}$ no modelo $\mathcal{M}_{k}$ segue uma distribuição bivariada normal assimétrica com matriz de correlações da seguinte forma

$$
\boldsymbol{R}=\left(\begin{array}{cc}
1 & \frac{\sigma_{12}}{\sqrt{\left(1+\delta_{k 1}^{2}\right)\left(1+\delta_{12}^{2}\right)}} \\
\frac{\sigma_{12}}{\sqrt{\left(1+\delta_{k 1}^{2}\right)\left(1+\delta_{k 2}^{2}\right)}} & 1
\end{array}\right) .
$$

É importante notar que as matrizes $\boldsymbol{\Gamma}$ e $\boldsymbol{R}$ definidas, respectivamente, em (5.5) e (5.6), não 
representam a estrutura de correlação entre as variáveis $Y_{i 1}$ e $Y_{i 2}$, a qual é obtida resolvendo a expressão (5.2) para o modelo probito assimétrico.

As distribuições a priori para os parâmetros de interesse consideradas para cada um dos modelos foram as mesmas apresentadas na seção anterior:

$$
\boldsymbol{\beta} \sim \mathcal{N}_{2}\left(\mathbf{0} ; 1000 \boldsymbol{I}_{7}\right),
$$

e a distribuição a priori em (3.30) para $\boldsymbol{\Sigma}$. A escolha destas distribuições a priori nos fornece distribuições a posteriori que podem ser simuladas através do algoritmo de Gibbs apresentado no Capítulo 3.

Durante o processo de simulação das amostras a posteriori dos parâmetros de interesse de cada um dos modelos, as primeiras 10.000 iterações são descartadas e saltamos de 10 em 10 iterações após o período de aquecimento para obter cadeias menos correlacionadas. Todos os algoritmos MCMC foram escritos usando a linguagem de programação matricial ox (Doornik, 2006). As convergências das cadeias geradas foram verificadas utilizando diversos procedimentos de diagnósticos incluídos no pacote CODA, por exemplo, o teste de Geweke e os gráficos do traço e das médias ergódicas (ver, Plummer et al., 2006). Ao final do processo a convergência foi alcançada e as inferências foram baseadas em amostras tamanho 2.000 para cada parâmetro.

A Tabela 5.3 apresenta as medidas de comparação de modelos descritas no Apêndice A.1 obtidas para cada um dos 40 modelos concorrentes. Note que o modelo bivariado probito $\left(\mathcal{M}_{1}\right)$ apresenta os menores valores de AIC, BIC, $\bar{D}$ e DIC. Além disto, observe que os valores apresentados pelos modelos com vetor de parâmetros de forma próximos do ponto $\boldsymbol{\delta}_{1}=(0,0)^{\prime}$ não estão muito distantes dos valores obtidos pelo modelo simétrico $\left(\mathcal{M}_{1}\right)$. A última coluna da A Tabela 5.3 apresenta o PsBF em favor do modelo $\mathcal{M}_{1}$ em relação aos demais modelos. Valores maiores que um indicam que o modelo $\mathcal{M}_{1}$ é preferível. Também pela Tabela 5.3, temos que os critérios LPML e PsBF indicam o modelo $\mathcal{M}_{2}\left(\boldsymbol{\delta}_{2}=(0,1 ; 0,0)^{\prime}\right)$ como melhor o ajuste. No entanto, Spiegelhalter et al. (2002) argumenta que uma diferença menor que 5 no DIC não faz, necessariamente, com que o modelo que apresenta menor DIC deve ser o escolhido. Outros critérios de seleção de modelos devem ser considerados nesta situação.

A Tabela 5.3 nos mostra que os modelos com parâmetros de assimetria distantes do ponto $(0,0)$ apresentam um ajuste mais pobre que os modelos próximos do ponto $(0,0)$. No entanto, estas medidas não mostram indícios suficientes para escolher entre os modelos com valores baixos para os parâmetros de assimetria.

A próxima seção apresenta uma análise de diagnóstico de observações influentes que é particularmente útil nos casos em que as medidas de ajuste não apresentam informações suficientes para selecionar um entre os modelos concorrentes. 
Tabela 5.3: Critérios de seleção $A I C, B I C, \bar{D}, p_{D}$, DIC, LPML e PsBF para os 40 modelos concorrentes aplicados no conjunto de dados sobre o comportamento de votos na cidade de Troy.

\begin{tabular}{|c|c|c|c|c|c|c|c|c|c|}
\hline $\mathcal{M}$ & $\delta_{1}$ & $\delta_{2}$ & $\mathrm{AIC}$ & $\mathrm{BIC}$ & $\bar{D}$ & $p_{D}$ & DIC & LPML & $\mathrm{PsBF}$ \\
\hline 1 & 0,00 & 0,00 & 210,94 & 231,37 & 194,94 & 8,41 & 211,76 & $-107,81$ & 1,00 \\
\hline 2 & 0,10 & 0,00 & 211,80 & 232,23 & 195,80 & 8,57 & 212,95 & $-107,39$ & 0,66 \\
\hline 3 & 0,00 & 0,10 & 212,40 & 232,83 & 196,40 & 8,38 & 213,17 & $-107,78$ & 0,98 \\
\hline 4 & $-0,10$ & 0,00 & 212,11 & 232,54 & 196,11 & 8,34 & 212,80 & $-108,20$ & 1,48 \\
\hline 5 & 0,00 & $-0,10$ & 212,32 & 232,75 & 196,32 & 8,55 & 213,44 & $-107,80$ & 0,99 \\
\hline 6 & $-0,10$ & $-0,10$ & 213,14 & 233,57 & 197,14 & 8,18 & 213,50 & $-108,10$ & 1,34 \\
\hline 7 & 0,10 & 0,10 & 212,83 & 233,26 & 196,83 & 8,48 & 213,79 & $-107,74$ & 0,94 \\
\hline 8 & 0,10 & $-0,10$ & 213,76 & 234,19 & 197,76 & 8,65 & 215,07 & $-108,50$ & 1,99 \\
\hline 9 & $-0,10$ & 0,10 & 214,17 & 234,60 & 198,17 & 8,52 & 215,23 & $-109,62$ & 6,10 \\
\hline 10 & 0,20 & 0,00 & 215,12 & 235,55 & 199,12 & 8,89 & 216,90 & $-109,19$ & 3,97 \\
\hline 11 & $-0,20$ & 0,00 & 215,35 & 235,78 & 199,35 & 8,23 & 215,83 & $-109,99$ & 8,89 \\
\hline 12 & 0,00 & $-0,20$ & 216,67 & 237,10 & 200,67 & 8,50 & 217,67 & $-110,42$ & 13,65 \\
\hline 13 & 0,00 & 0,20 & 216,75 & 237,18 & 200,75 & 8,75 & 218,26 & $-110,69$ & 17,78 \\
\hline 14 & 0,10 & $-0,20$ & 218,42 & 238,85 & 202,42 & 8,67 & 219,77 & $-111,07$ & 26,02 \\
\hline 15 & 0,20 & $-0,10$ & 217,60 & 238,03 & 201,60 & 9,05 & 219,71 & $-110,95$ & 23,11 \\
\hline 16 & $-0,10$ & 0,20 & 218,93 & 239,36 & 202,93 & 8,83 & 220,60 & $-113,39$ & $>99,99$ \\
\hline 17 & $-0,20$ & 0,10 & 217,65 & 238,08 & 201,65 & 8,69 & 219,04 & $-137,32$ & $>99,99$ \\
\hline 18 & $-0,20$ & $-0,20$ & 218,94 & 239,37 & 202,94 & 8,16 & 219,27 & $-111,41$ & 36,79 \\
\hline 19 & 0,20 & 0,20 & 218,76 & 239,19 & 202,76 & 9,12 & 221,02 & $-111,47$ & 38,76 \\
\hline 21 & 0,00 & $-0,30$ & 215,81 & 236,24 & 199,81 & 8,12 & 216,07 & $-109,03$ & 6,84 \\
\hline 22 & $-0,30$ & 0,00 & 215,49 & 235,93 & 199,49 & 8,37 & 216,25 & $-110,01$ & 9,10 \\
\hline 23 & $-0,30$ & $-0,30$ & 228,57 & 249,00 & 212,57 & 8,06 & 228,71 & $-117,29$ & $>99,99$ \\
\hline 24 & 0,30 & 0,30 & 228,50 & 248,93 & 212,50 & 9,40 & 231,30 & $-116,88$ & $>99,99$ \\
\hline 25 & 0,30 & $-0,30$ & 237,72 & 258,15 & 221,72 & 10,54 & 242,81 & $-143,97$ & $>99,99$ \\
\hline 26 & $-0,30$ & 0,30 & 236,50 & 256,93 & 220,50 & 10,14 & 240,79 & $-175,22$ & $>99,99$ \\
\hline 27 & 0,20 & $-0,20$ & 222,42 & 242,85 & 206,42 & 9,33 & 225,08 & $-114,36$ & $>99,99$ \\
\hline 28 & $-0,20$ & 0,20 & 222,49 & 242,92 & 206,49 & 9,29 & 225,07 & $-134,74$ & $>99,99$ \\
\hline 29 & 0,30 & 0,00 & 220,25 & 240,68 & 196,11 & 9,36 & 222,97 & $-112,62$ & $>99,99$ \\
\hline 30 & 0,00 & 0,30 & 223,69 & 244,12 & 196,32 & 9,19 & 226,07 & $-117,96$ & $>99,99$ \\
\hline 31 & 0,50 & 0,00 & 237,64 & 258,07 & 199,12 & 10,14 & 241,91 & $-125,69$ & $>99,99$ \\
\hline 32 & 0,00 & 0,50 & 222,08 & 242,51 & 195,80 & 8,99 & 224,06 & $-113,01$ & $>99,99$ \\
\hline 33 & 0,00 & $-0,50$ & 223,96 & 244,29 & 207,96 & 8,35 & 224,67 & $-115,08$ & $>99,99$ \\
\hline 34 & $-0,50$ & 0,00 & 222,78 & 243,21 & 206,78 & 8,49 & 223,78 & $-114,03$ & $>99,99$ \\
\hline 35 & $-0,50$ & $-0,50$ & 256,10 & 276,53 & 240,10 & 8,69 & 257,49 & $-133,48$ & $>99,99$ \\
\hline 36 & 0,50 & 0,50 & 258,40 & 278,83 & 242,40 & 10,61 & 263,63 & $-134,23$ & $>99,99$ \\
\hline 37 & 0,00 & 1,00 & 249,53 & 269,96 & 196,40 & 10,18 & 253,88 & $-128,27$ & $>99,99$ \\
\hline 38 & 1,00 & 0,00 & 307,76 & 328,19 & 200,75 & 16,70 & 325,17 & $-227,17$ & $>99,99$ \\
\hline 39 & 1,00 & 1,00 & 354,04 & 374,47 & 338,04 & 11,24 & 360,52 & $-195,17$ & $>99,99$ \\
\hline 40 & $-1,00$ & $-1,00$ & 363,64 & 384,07 & 347,64 & 13,41 & 374,46 & $-193,85$ & $>99,99$ \\
\hline
\end{tabular}

\subsection{Diagnóstico de influência}

Ao se ajustar um modelo a um conjunto de dados, é imprescindível que os resultados obtidos a partir do modelo proposto sejam resistentes a pequenas perturbações, tanto no modelo como nos dados. Caso isto não ocorra, o modelo pode conduzir a inferências errôneas. Logo, é importante a realização de um estudo de robustez dos modelos a perturbações nos dados. A análise de diagnóstico 
de exclusão de casos pode ser utilizada com este fim.

Nesta seção são discutidos e aplicados os procedimentos de diagnóstico de influência descritos no Apêndice A.2 em 12 modelos selecionados entre os 40 comparados na seção anterior. Foram selecionados os 11 modelos que obtiveram os melhores ajustes e o modelo que obteve o pior ajuste (ver Tabela 5.3). O modelo que obteve o pior ajuste foi selecionado com o objetivo de verificar o comportamento destas medidas em modelos pobremente ajustados. Os modelos que apresentaram os melhores ajuste foram os modelos $\mathcal{M}_{1}, \mathcal{M}_{2}, \mathcal{M}_{3}, \mathcal{M}_{4}, \mathcal{M}_{5}, \mathcal{M}_{6}, \mathcal{M}_{7}, \mathcal{M}_{8}, \mathcal{M}_{9}, \mathcal{M}_{10}$ e $\mathcal{M}_{21}$. O modelo que apresentou o pior ajuste foi o modelo $\mathcal{M}_{40}$.

Não existe na literatura sugestões de pontos de corte para identificação de observações influentes através das medidas CPO e divergência K-L (ver Apêndice A.2). Diferentes pontos de corte foram considerados por Chen et al. (2008), Cancho, Ortega e Paula (2010) e Farias e Lemonte (2011) em suas aplicações. Aqui nós consideraremos como pontos influentes aquelas observações que apresentarem valores destas medidas significativamente maiores que os valores apresentados pelas demais observações do conjunto de dados.

Os gráficos do índice das observações contra os valores do inverso da $\mathrm{CPO}\left(\mathrm{CPO}^{-1}\right)$ para os 12 modelos analisados são mostrados nas Figuras 5.4 e 5.5. Uma linha tracejada é desenhada no valor 15 para auxiliar na visualização de diferenças entre os valores observados desta medida. Observações com $\mathrm{CPO}^{-1}$ maiores que 15 são marcados neste gráfico e considerados influentes neste estudo. Note que as observações \#36, \#59, \#61, \#63, \#72 e \#95 apresentam valores altos para a maioria dos modelos analisados, em particular, para o modelo que apresentou o pior ajuste (ver Figura 5.5 (f)), neste caso, vários valores chegam a ser maiores que 1.000. A Figura 5.4 (a) mostra que estas mesmas observações também são marcadas como influentes no modelo $\mathcal{M}_{1}(\boldsymbol{\delta}=(0,0 ; 0,0))$. A Figura 5.5 (c) mostra que o modelo $\mathcal{M}_{10}(\boldsymbol{\delta}=(0,0 ; 0,2))$ foi o único modelo que não apresentou observações influentes.

As Figuras 5.6 e 5.7 apresentam os gráficos do índice das observações contra os valores da divergência K-L para todos os modelos analisados. Uma linha tracejada é desenhada no valor 0,3 para auxiliar na visualização de diferenças entre os valores obtidos. Casos com a divergência K-L maiores que 0,3 são marcados neste gráfico e considerados influentes. Analisando a Figura 5.6 (a) observamos que os casos \#36, \#43, \#59, \#74 e \#95 são considerados influentes para o modelo bivariado probito $(\boldsymbol{\delta}=(0,0 ; 0,0))$ de acordo com a divergência K-L. Destas, as observações \#43 e \#74 são consideradas influentes na maioria dos modelos analisados, incluindo o modelo $\mathcal{M}_{10}$ $(\boldsymbol{\delta}=(0,0 ; 0,2))$, apesar de não apresentar um valor tão alto da divergência K-L como nos outros modelos. O modelo $\mathcal{M}_{10}(\boldsymbol{\delta}=(0,0 ; 0,2))$ é o modelo que apresenta menos observações influentes de acordo com as medidas $\mathrm{CPO}^{-1}$ e divergência K-L. Por este motivo, focaremos daqui em diante o estudo na comparação entre os modelos $\mathcal{M}_{1}$ e $\mathcal{M}_{10}$.

A Figura 5.8 apresentam os gráficos do índice das observações contra os valores da calibração da divergência K-L $\left(p_{i}\right)$ para os modelos $\mathcal{M}_{1}$ e $\mathcal{M}_{10}$. Uma linha tracejada é desenhada no valor 0,85 . Observações com $p_{i}$ maiores que 0,85 são marcados e considerados influentes. 
A Tabela 5.4 lista as observações marcadas como influentes em alguns dos critérios definidos anteriormente $\left(\mathrm{CPO}^{-1}>15, K L>0,3\right.$ ou $\left.p_{i}>0,85\right)$ no modelo $\mathcal{M}_{1}$. Para realização de uma comparação entre modelos, esta tabela também apresenta os valores destas medidas para o modelo $\mathcal{M}_{10}$. Analisando esta tabela notamos que as observações listadas são bem mais influentes no modelo $\mathcal{M}_{1}$ que no modelo $\mathcal{M}_{10}$. Mostrando que o modelo $\mathcal{M}_{10}$ é menos sensível (mais robusto) à mudanças no conjunto de dados .

Tabela 5.4: Valores das medidas de influência das observações influentes para o modelo $\mathcal{M}_{1}$ comparada com as valores obtidos pelo modelo $\mathcal{M}_{10}$ (c indica que a observação é influente segundo a CPO e $k$ indica que a observação é influente de acordo com a divergência $K-L$ ).

\begin{tabular}{lccccccc}
\hline & \multicolumn{3}{c}{$\mathcal{M}_{1}$} & & \multicolumn{3}{c}{$\mathcal{M}_{11}$} \\
\cline { 2 - 3 } \cline { 6 - 7 } obs & $C P O_{i}^{-1}$ & $K\left(\pi, \pi_{(-i)}\right)$ & $p_{i}$ & & $C P O_{i}^{-1}$ & $K\left(\pi, \pi_{(-i)}\right)$ & $p_{i}$ \\
\hline $36^{c, k}$ & 32,95 & 0,54 & 0,91 & & 11,53 & 0,09 & 0,71 \\
$43^{k}$ & 8,62 & 0,35 & 0,85 & & 8,62 & 0,33 & 0,84 \\
$59^{c, k}$ & 32,95 & 0,54 & 0,91 & & 11,53 & 0,09 & 0,71 \\
$61^{c}$ & 23,33 & 0,19 & 0,78 & & 14,81 & 0,14 & 0,75 \\
$65^{c}$ & 18,80 & 0,17 & 0,77 & & 9,92 & 0,11 & 0,72 \\
$72^{c, k}$ & 22,29 & 0,24 & 0,81 & & 12,08 & 0,17 & 0,77 \\
$74^{k}$ & 11,03 & 0,35 & 0,85 & & 7,46 & 0,32 & 0,84 \\
$95^{c, k}$ & 15,10 & 0,59 & 0,92 & & 7,60 & 0,30 & 0,83 \\
\hline
\end{tabular}

Analisando a Tabela 5.5 notamos que a maioria das observações marcadas como influentes de acordo com a CPO (\#36, \#59, \#65 e \#95) são referentes a indivíduos que não tem filhos na escola pública, mas votaram a favor do fundo orçamentário. Pela sexta e a sétima coluna desta tabela, observamos que o modelo $\mathcal{M}_{1}$ fornece uma probabilidade ajustada para este evento menor do que a probabilidade ajustada apresentada pelo modelo $\mathcal{M}_{10}$. Isto indica que pessoas que não tem crianças na escola pública são mais propensas a votar no fundo orçamentário que o esperando quando o modelo assimétrico é considerado. As probabilidades ajustadas chegam próximas a duplicar o valor no modelo $\mathcal{M}_{10}$ na maioria dos casos influentes. Estas observações, com exceção do caso \#65, também foram identificadas pela divergência K-L e calibradas com valores maiores que 0,90 no modelo $\mathcal{M}_{1}$, que é bem maior que 0,5 .

Tabela 5.5: Observações influentes para o modelo $\mathcal{M}_{1}$ e suas respectivas probabilidades ajustadas para os modelos $\mathcal{M}_{1}$ e $\mathcal{M}_{10}$ (c indica que a observação é influente segundo a $\mathrm{CPO}^{-1}$ e $k$ indica que a observação é influente de acordo com a divergência $K-L$ ).

\begin{tabular}{lccccccc}
\hline & $y_{1}$ & $y_{2}$ & INC & TAX & YRS & $P\left(\boldsymbol{Y}_{i}=\boldsymbol{y}_{i} \mid \mathcal{M}_{1}\right)$ & $P\left(\boldsymbol{Y}_{i}=\boldsymbol{y}_{i} \mid \mathcal{M}_{10}\right)$ \\
\hline $36^{c, k}$ & 0 & 1 & 10,22 & 7,05 & 5 & 0,0592 & 0,1034 \\
$43^{k}$ & 0 & 0 & 8,92 & 5,99 & 6 & 0,1649 & 0,2000 \\
$59^{c, k}$ & 0 & 1 & 10,22 & 7,05 & 5 & 0,0592 & 0,1034 \\
$61^{c}$ & 0 & 0 & 10,46 & 7,05 & 7 & 0,0602 & 0,0875 \\
$65^{c}$ & 0 & 1 & 10,46 & 7,05 & 4 & 0,0724 & 0,1233 \\
$72^{c, k}$ & 0 & 0 & 10,22 & 7,05 & 18 & 0,0688 & 0,1134 \\
$74^{k}$ & 1 & 1 & 9,43 & 6,75 & 49 & 0,2470 & 0,2247 \\
$95^{c, k}$ & 0 & 1 & 8,92 & 5,99 & 35 & 0,1591 & 0,2153 \\
\hline
\end{tabular}


Estes resultados mostraram que o modelo bivariado é bem mais sensível a observações influentes que o modelo bivariado probito assimétrico com parâmetro de forma $\boldsymbol{\delta}=(0,0 ; 0,2)^{\prime}$. A Tabela 5.6 apresenta as estatísticas a posteriori do ajuste do modelo probito assimétrico com vetor de parâmetros de forma $\boldsymbol{\delta}=(0,0 ; 0,2)$.

As estimativas pontuais das correlações $\rho\left(Y_{i 1}, Y_{i 2}\right)$ para cada indivíduo pertencente ao conjunto de dados ao se considerar o modelo probito assimétrico com vetor de parâmetros de forma $\boldsymbol{\delta}=$ $(0,0 ; 0,2)$ são apresentadas na Figura 5.3 (a). A Figura 5.3 (b) apresenta os gráficos boxplots das distribuições a posteriori das correlações para cada indivíduo. Uma linha tracejada é desenhada em ambos os graficos na média das correlações das variáveis resposta. A correlação média é igual 0,2108 e o desvio-padrão médio é dado por 0,1278 . Estes resultados indicam que existe uma correlação de aproximadamente 0,21 entre as variáveis $Y_{1}$ e $Y_{2}, i=1, \ldots, n$.

Tabela 5.6: Estatísticas a posteriori para o modelo bivariado probito assimétrico com vetor de parâmetros de forma $\boldsymbol{\delta}=(0,0 ; 0,2)^{\prime}$ ajustado ao conjunto de dados sobre o comportamento de votos na cidade de Troy.

\begin{tabular}{rrrrrrrrr}
\hline & & & & & \multicolumn{2}{c}{ HPD de 90\% } & \multicolumn{2}{c}{ HPD de 95\% } \\
\multicolumn{2}{r}{ Parâmetro } & Média & D. P. & Mediana & Inferior & Superior & Inferior & Superior \\
\hline $\boldsymbol{\beta}_{1}$ & $\beta_{11}$ & $-4,9414$ & 3,9512 & $-4,97147$ & $-11,1833$ & 1,7562 & $-13,0750$ & 2,2026 \\
& $\beta_{12}$ & 0,0974 & 0,4532 & 0,10361 & $-0,6403$ & 0,8457 & $-0,8209$ & 0,9660 \\
& $\beta_{13}$ & 0,6998 & 0,5752 & 0,69377 & $-0,3208$ & 1,5775 & $-0,3633$ & 1,8244 \\
$\boldsymbol{\beta}_{2}$ & $\beta_{21}$ & $-0,2770$ & 4,0643 & $-0,21890$ & $-7,1979$ & 6,1091 & $-7,8126$ & 7,8337 \\
& $\beta_{22}$ & 1,0268 & 0,4428 & 1,01513 & 0,2401 & 1,6880 & 0,1641 & 1,8944 \\
& $\beta_{23}$ & $-1,3649$ & 0,5815 & $-1,34837$ & $-2,2515$ & $-0,3877$ & $-2,4351$ & $-0,2609$ \\
& $\beta_{24}$ & $-0,0178$ & 0,0148 & $-0,01813$ & $-0,0414$ & 0,0070 & $-0,0453$ & 0,0111 \\
$\sigma_{12}$ & & 0,3744 & 0,2131 & 0,39088 & 0,0333 & 0,7236 & $-0,0394$ & 0,7553 \\
\hline
\end{tabular}

(a)

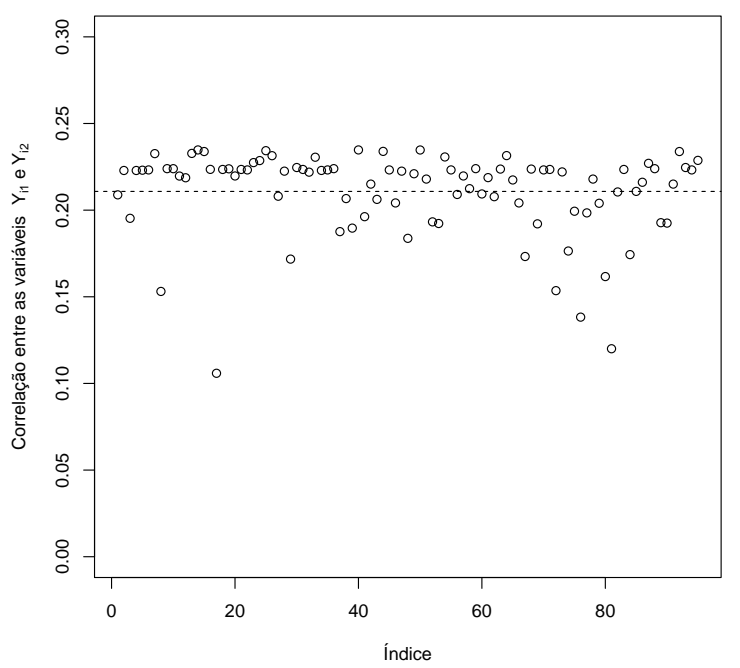

(b)

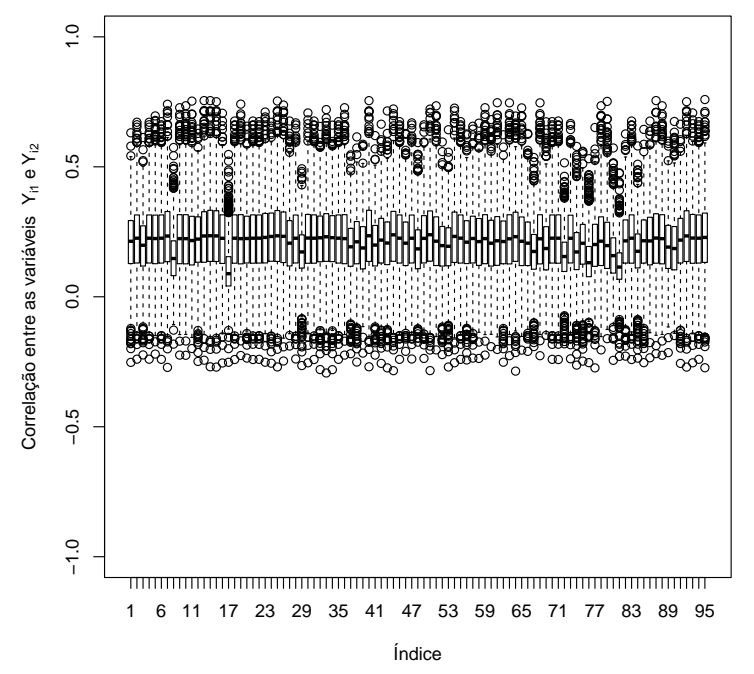

Figura 5.3: Estimativas pontuais das correlações (a) e gráficos boxplot das distribuições a posteriori das correlações (b) considerando o modelo probito assimétrico. 


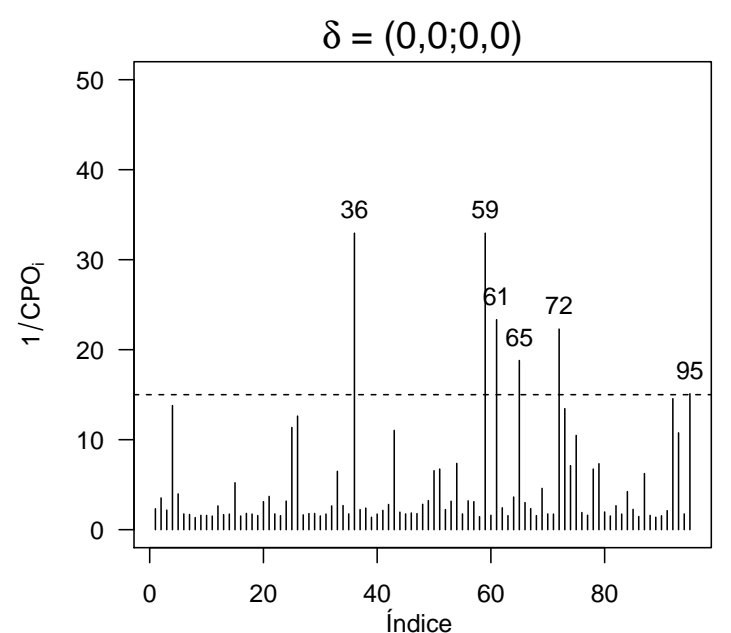

(a)

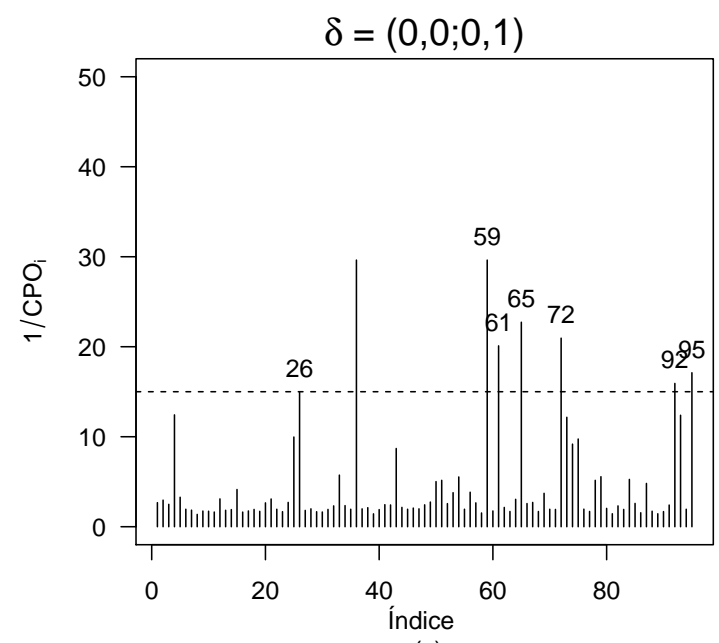

(c)

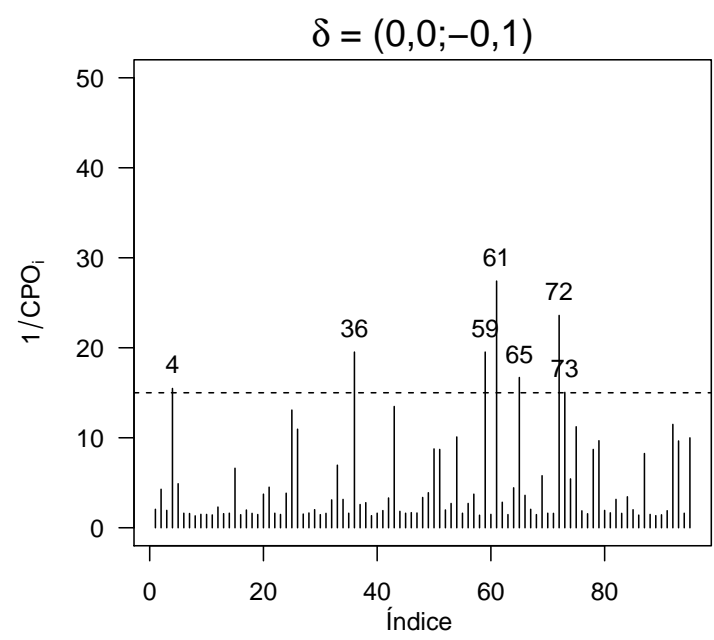

(e)

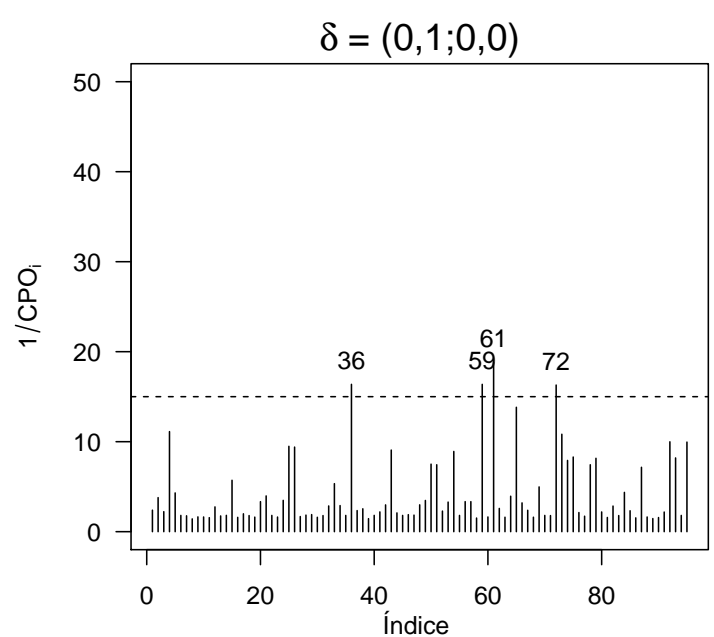

(b)

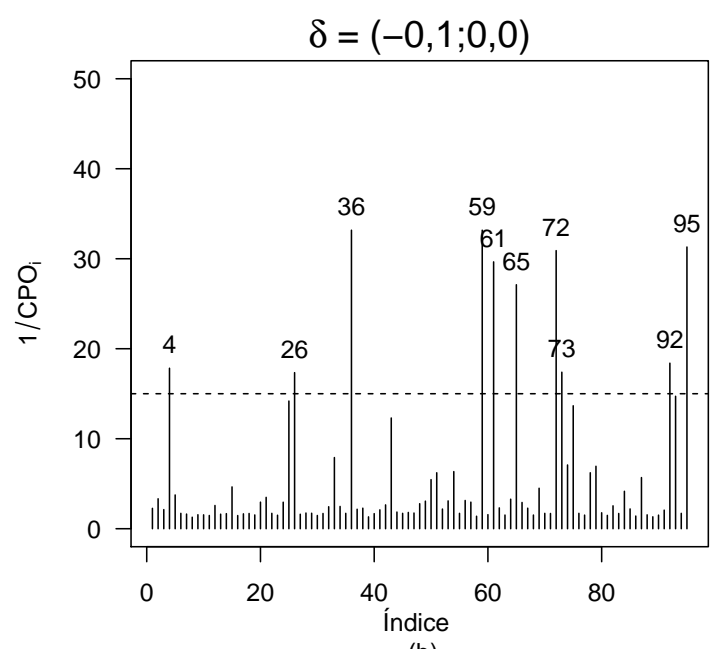

(b)

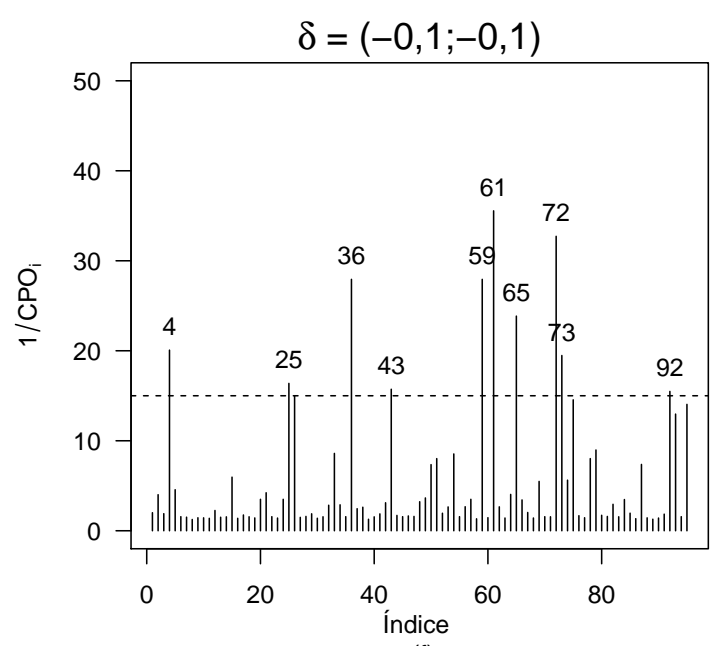

(f)

Figura 5.4: $O$ inverso do CPO aplicado ao conjunto de dados sobre o comportamento de votos na cidade de Troy para os modelos $\mathcal{M}_{1}, \mathcal{M}_{2}, \mathcal{M}_{3}, \mathcal{M}_{4}, \mathcal{M}_{5}$ e $\mathcal{M}_{6}$. 


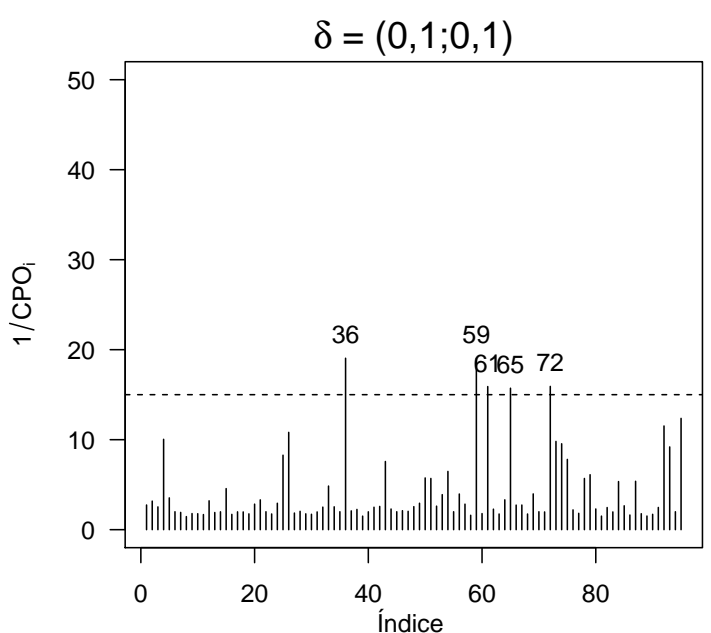

(a)

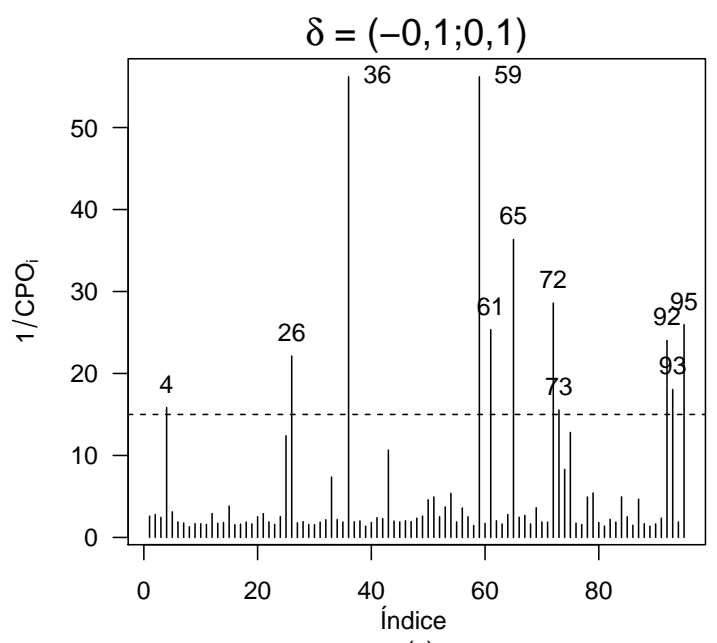

(c)

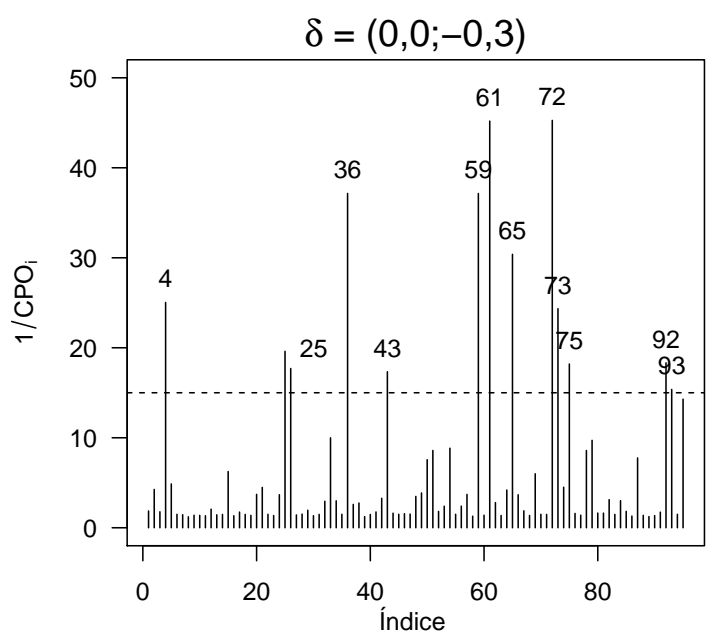

(e)

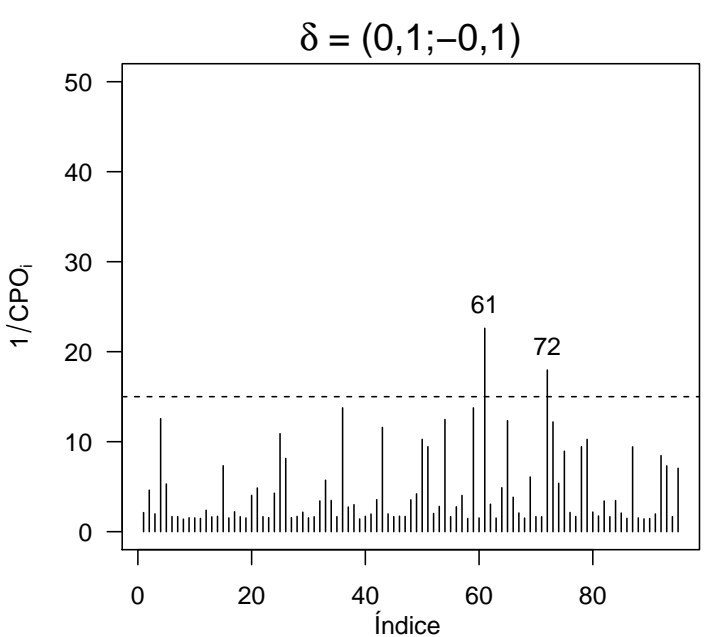

(d)

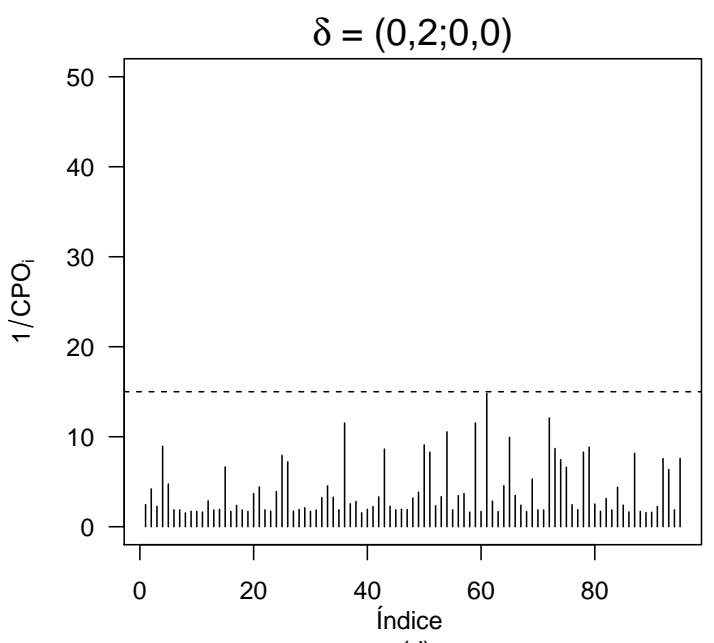

(d)

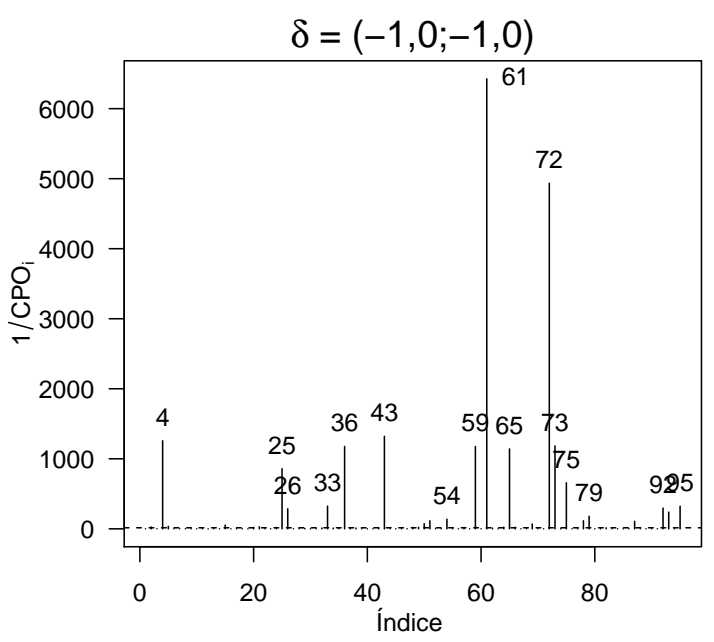

(f)

Figura 5.5: $O$ inverso do CPO aplicado ao conjunto de dados sobre o comportamento de votos na cidade de Troy para os modelos $\mathcal{M}_{7}, \mathcal{M}_{8}, \mathcal{M}_{9}, \mathcal{M}_{10}, \mathcal{M}_{21}$ e $\mathcal{M}_{40}$. 


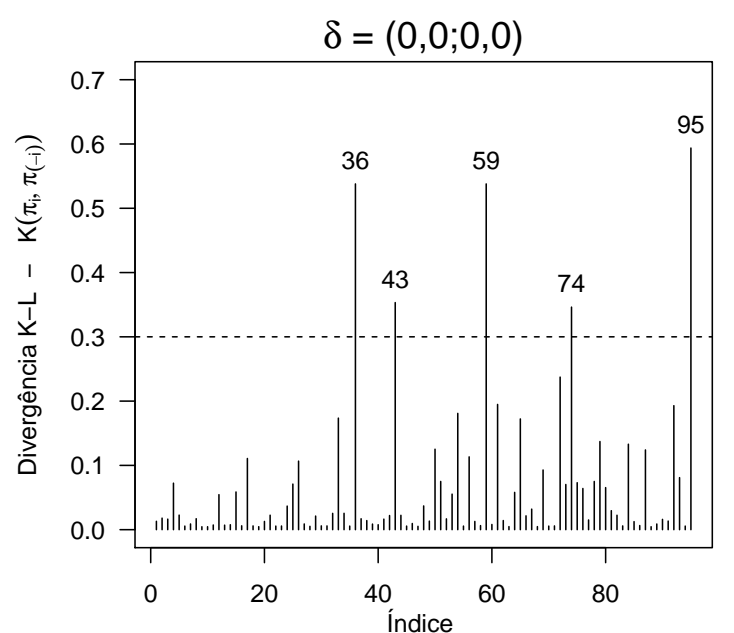

(a)

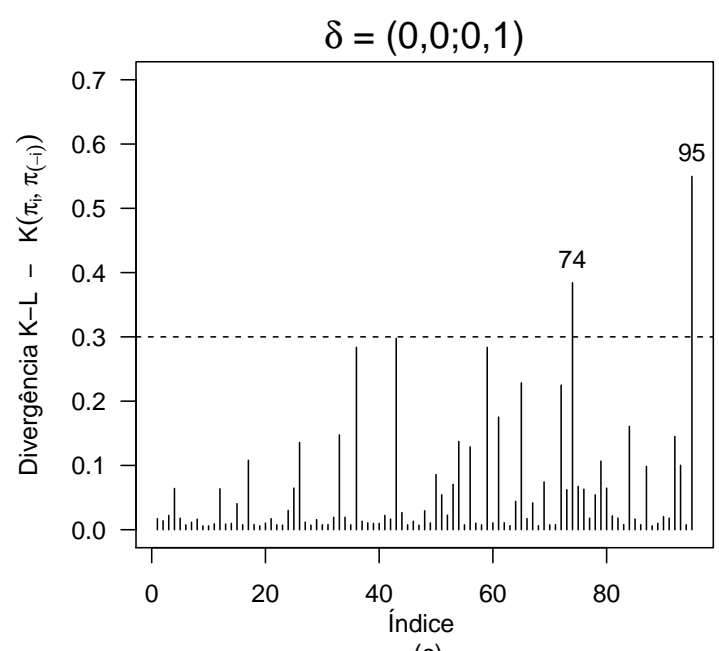

(c)

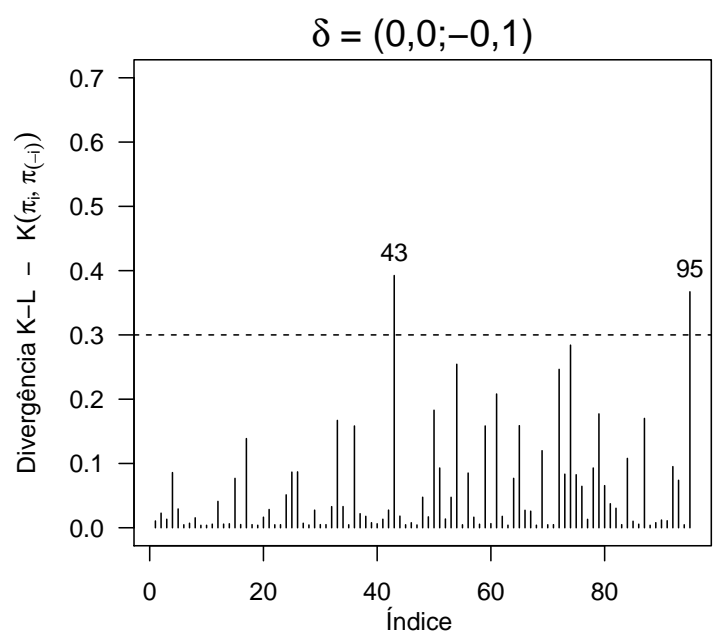

(e)

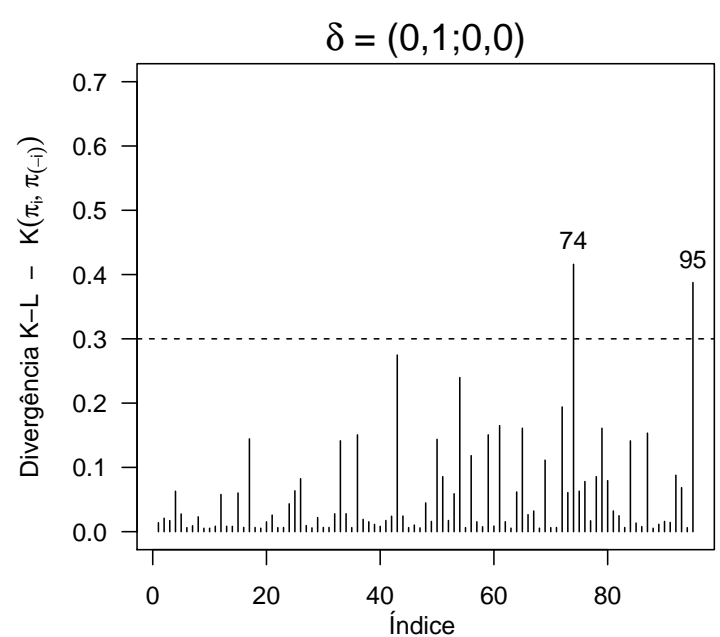

(b)

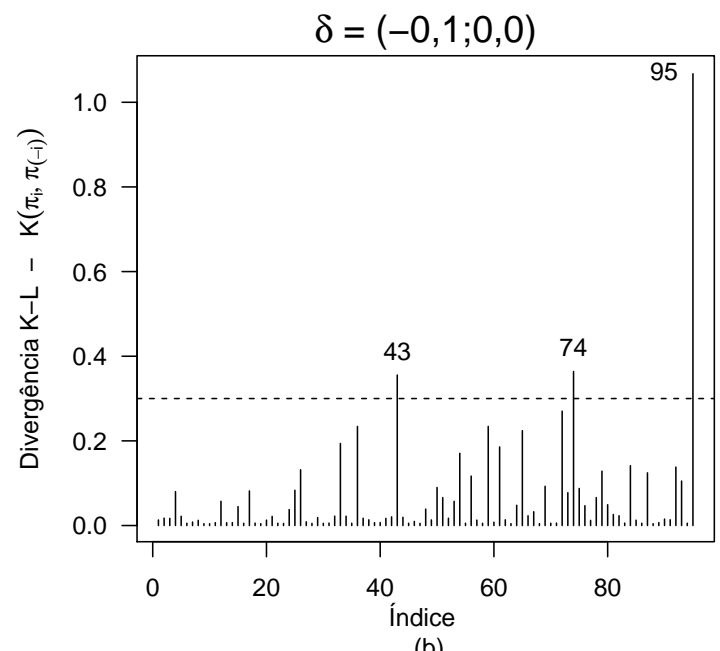

(b)

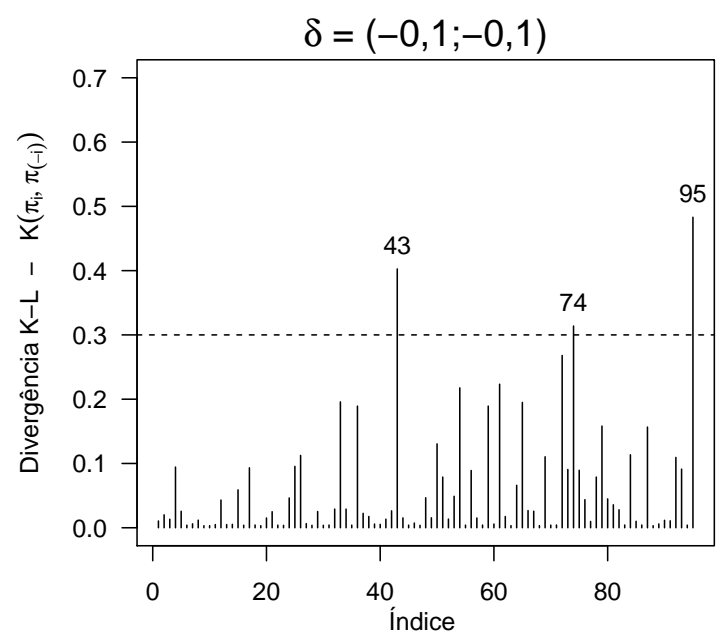

(f)

Figura 5.6: A divergência $K-L$ aplicada ao conjunto de dados sobre o comportamento de votos na cidade de Troy para os modelos $\mathcal{M}_{1}, \mathcal{M}_{2}, \mathcal{M}_{3}, \mathcal{M}_{4}, \mathcal{M}_{5}$ e $\mathcal{M}_{6}$. 


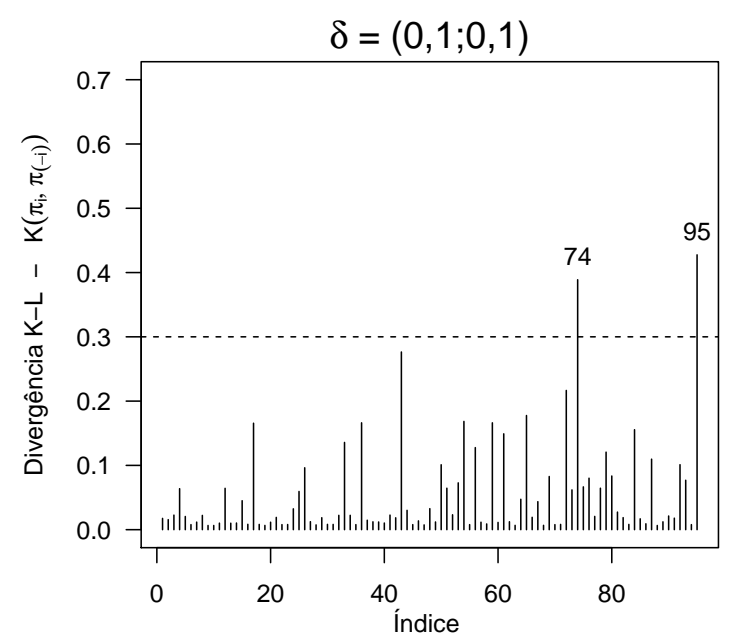

(a)

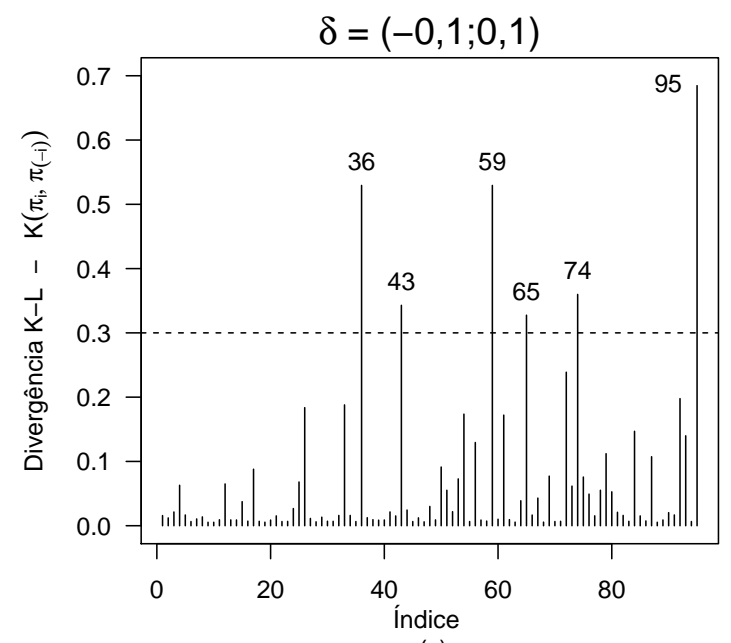

(c)

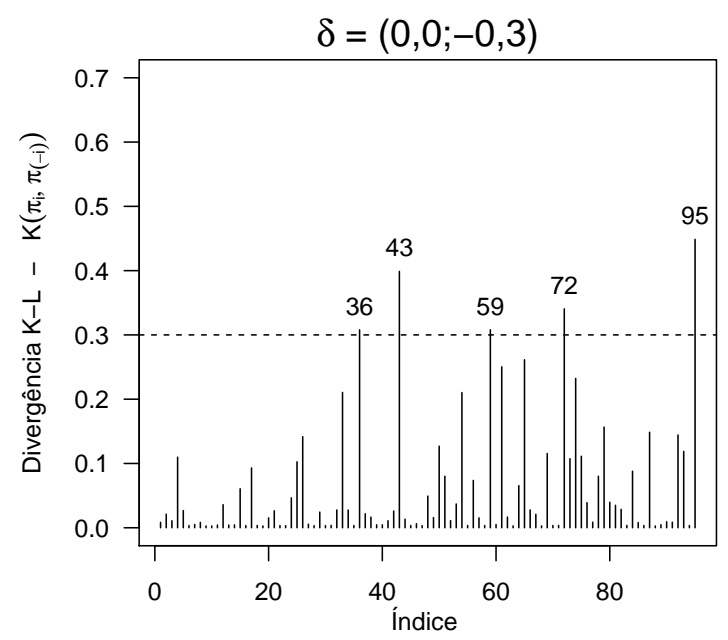

(e)

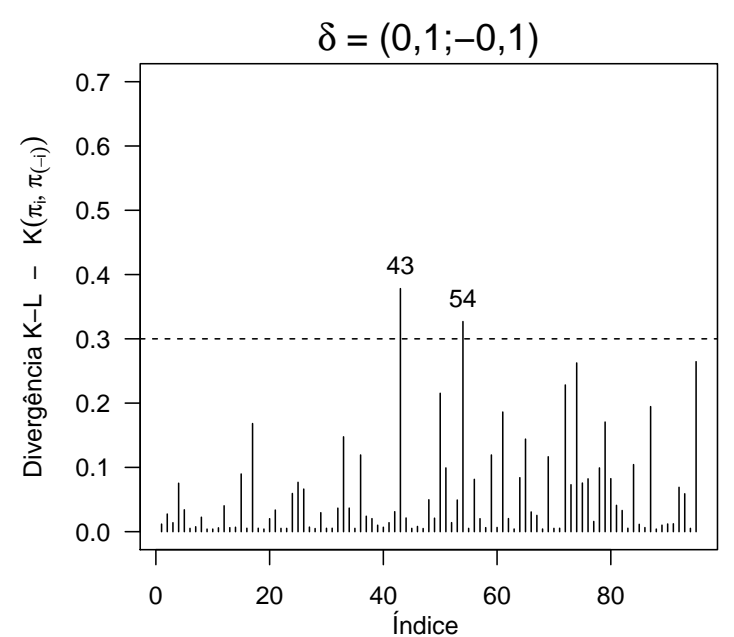

(d)

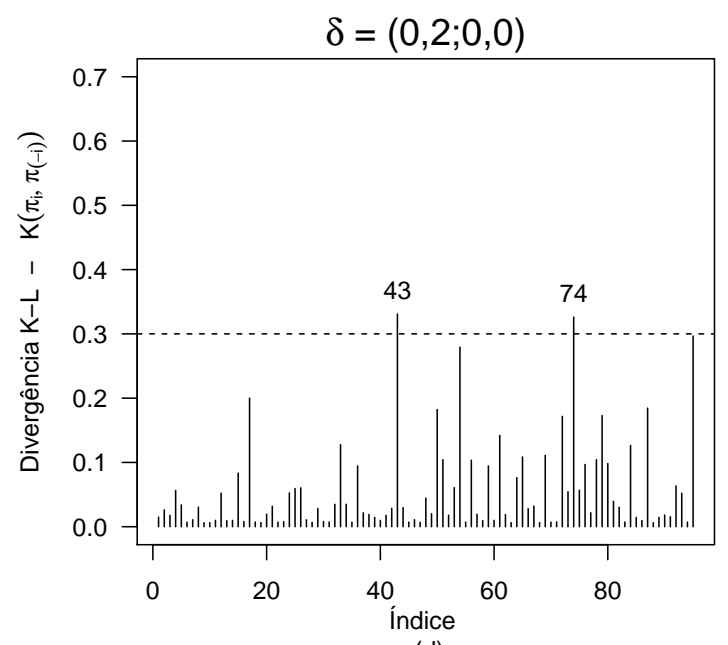

(d)

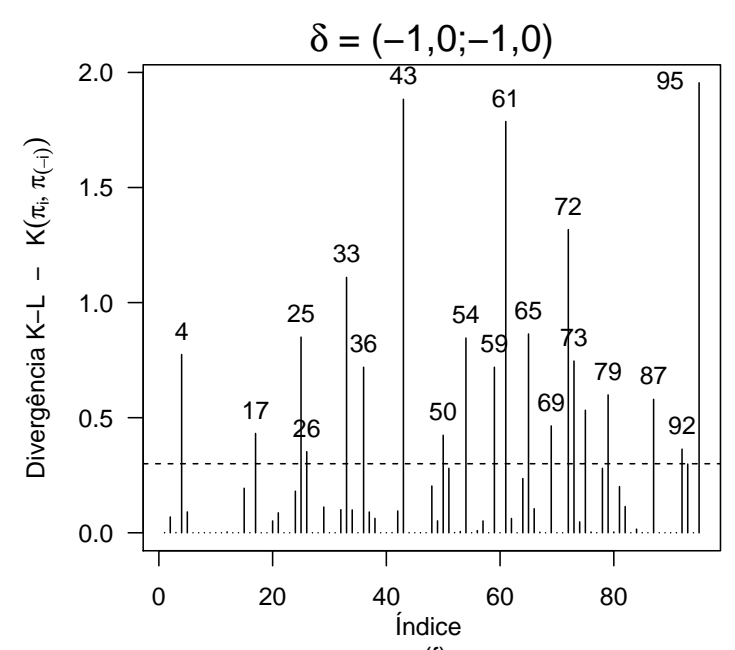

(f)

Figura 5.7: A divergência $K-L$ aplicada ao conjunto de dados sobre o comportamento de votos na cidade de Troy para os modelos $\mathcal{M}_{7}, \mathcal{M}_{8}, \mathcal{M}_{9}, \mathcal{M}_{10}, \mathcal{M}_{21}$ e $\mathcal{M}_{40}$. 


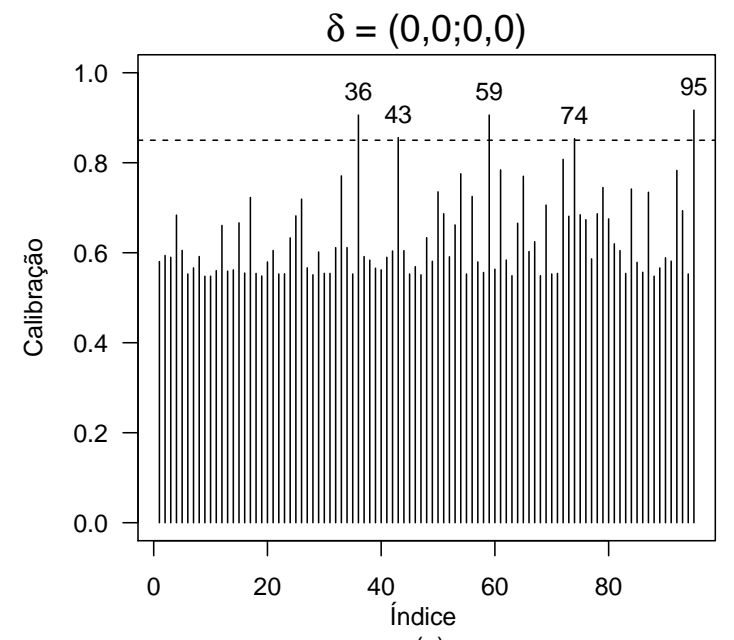

(a)

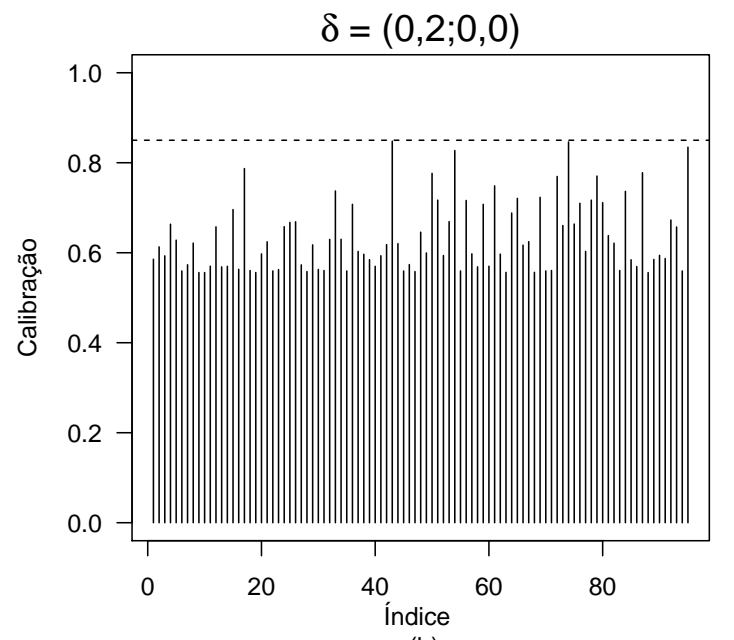

(b)

Figura 5.8: A calibração da divergência $K$-L aplicada ao ao conjunto de dados sobre o comportamento de votos na cidade de Troy para os modelos $\mathcal{M}_{1}$ e $\mathcal{M}_{11}$. 


\section{Capítulo 6}

\section{Comentários Finais}

Nesta tese introduzimos uma classe de modelos de regressão multivariada com função de ligação assimétrica para o ajuste de conjuntos de dados com respostas multivariadas binárias. Esta classe de modelos é obtida considerando que as funções de ligação do modelo de regressão para resposta binária pertence à classe de distribuições elípticas assimétricas proposta por Sahu, Dey e Branco (2003). Esta classe de modelos é bastante rica, contemplando, além dos modelos multivariados probito (Chib e Greenberg, 1998) e logito (Chen e Dey, 2000), os modelos com funções de ligação elípticas, em particular os representáveis através de mistura no parâmetro de escala de distribuições normais multivariadas (Chen e Dey, 1998), como casos particulares.

Nós mostramos no Capítulo 3 que o modelo multivariado com função de ligação pertencente à classe de distribuições representáveis através de mistura no parâmetro de escala de distribuições multivariadas normais assimétricas (MENA) é não identificável quando nenhuma restrição é imposta para o espaço paramétrico do modelo. Para evitar este problema de falta de identificabilidade, nós sugerimos assumir que todos os elementos da diagonal principal da matriz de dispersão são iguais a 1 (um) em modelos simétricos e, além disto, sugeremos fixar os parâmetros de forma nos modelos assimétricos. É importante notar que estes resultados sobre falta de identificabilidade são úteis tanto na abordagem frequentísta quanto na abordagem bayesiana.

Para realizar inferências nesta classe de modelos utilizamos a abordagem bayesiana e desenvolvemos vários algoritmos de Monte Carlo via Cadeias de Markov (MCMC), em particular o algoritmo de Gibbs, para simular valores a posteriori dos parâmetros de interesse. Dois estudos de simulação foram desenvolvidos no Capítulo 4. No primeiro estudo verificamos que a estatística média a posteriori, obtida através das cadeias simuladas pelos algoritmos MCMC desenvolvidos no Capítulo 3, fornece boas estimativas pontuais para os parâmetros de interesse do modelo, com sua precisão aumentando com o aumento da amostra. No segundo estudo notamos que a escolha da função de ligação é importante e, caso seja mal especificada, pode fornecer ajustes pobres.

No Capítulo 5 ajustamos diversos modelos pertencentes à classe MENA em um conjunto de dados reais. Comparamos a qualidade dos ajustes utilizando as medidas de comparações de modelos descritas no Apêndice A.1 e realizamos uma análise de diagnóstico de influencia baseada nas medidas de influência descritas no Apêndice A.2. Por fim, verificamos que o modelo bivariado probito assimétrico foi o modelo selecionado, pois apresentou um bom ajuste e foi o modelo mais robusto 
a observações influentes.

As convergências das cadeias geradas pelos algoritmos MCMC foram verificadas utilizando diversos procedimentos de diagnósticos incluídos no pacote CODA (Plummer et al., 2006) e a linguagem de programação $\mathrm{R}$ foi utilizada para o cálculo das medidas de qualidade de ajuste e de diagnóstico de influência descritas no Apêndice A. É importante destacar que o comando pmnorm () incluído no pacote mnormt (Genz e Azzalini, 2011) foi utilizado para o calculo das funções de distribuições acumuladas das distribuições normais multivariadas necessárias. Os pacotes CODA e mnormt estão disponível para instalação no software estatístico R (R Development Core Team, 2012). Todos os códigos poderiam ter sido escritos somente na linguagem de programação R. No entanto, verificouse que os estudos de simulação descritos no Capítulo 4 tornavam-se inviáveis de serem realizados neste software devido sua demora para processar os algoritmos MCMC apresentados no Capítulo 3. Por esta razão e por apresentar um eficiente suporte estatístico o software Ox foi escolhido neste caso. Logo, os códigos dos algoritmos MCMC utilizados nos estudos de simulação do Capítulo 4 e na aplicação do Capítulo 5 foram escritos na linguagem de programação matricial ox (Doornik, 2006). Os programas e o banco de dados utilizados nesta tese podem ser solicitados via e-mail: rfarias@ime.usp.br

Este trabalho oferece várias possibilidades para futuras pesquisas, entre estas podemos destacar:

- Resolver o problema de falta de identificabilidade considerando outras restrições para o espaço paramétrico além das consideradas nesta tese.

- Desenvolver metodologias inferenciais frequentístas para os modelos multivariados com funções de ligação elípticas assimétricas.

- Desenvolver modelos multivariados com funções de ligação elípticas assimétricas para o ajuste de conjuntos de dados com respostas correlacionadas ordinais ou categóricas. 


\section{Apêndice A}

\section{Seleção de Modelos}

No contexto Bayesiano há vários critérios de seleção de modelos que podem ser utilizados para verificar, entre diversos modelos competidores, qual fornece o melhor ajuste aos dados. Neste apêndice apresentamos algumas medidas e discutiremos como elas podem ser utilizadas para este fim. As medidas consideradas são: o critério de informação de Akaike (AIC); o critério de informação de Bayesiano (BIC); o critério de informação da deviance (DIC); o logaritmo da pseudo-verossimilhança marginal (LPML); e o pseudo-Fator de Bayes (PsBF).

Adicionalmente, nós também apresentamos aqui algumas medidas que podem ser utilizadas para realização de análise de diagnóstico de observações influentes nos modelos. As medidas consideradas são: a ordenada preditiva condicional (CPO); a divergência de Kullback-Leibler (K-L divergência); e a divergência de Kullback-Leibler calibrada.

As medidas de seleção de modelos e de análise de diagnóstico de influência descritos neste apêndice para o modelo multivariado binário com função de ligação pertencente à classe de distribuições de mistura de escala normal assimétrica (MENA) podem ser obtidas através das amostras a posteriori dos parâmetros de interesse geradas pelos algoritmos de Monte Carlo via Cadeias de Markov (MCMC) apresentados no Capítulo 3.

\section{A.1 Medidas de comparação de modelos}

A função deviance tem um importante papel em comparação de modelos estatísticos devido sua conexão com a medida de Informação de Kullback-Leibler (Gelman et al., 2003). Isto faz com que ela seja utilizada em diversas medidas de seleção de modelos. A função deviance para o modelo de regressão binária com função de ligação pertencente à família de distribuições de mistura de escala normal assimétrica (MENA) é definida como,

$$
D(\boldsymbol{\theta} ; \boldsymbol{y})=-2 \log [L(\boldsymbol{\theta} ; \boldsymbol{y})]
$$

em que $\boldsymbol{\theta}=(\boldsymbol{\beta}, \boldsymbol{\sigma}, \boldsymbol{\delta})$ é o vetor de parâmetros de interesse e

$$
L(\boldsymbol{\theta} ; \boldsymbol{y})=\prod_{i=1}^{n} P\left(\boldsymbol{Y}_{i}=\boldsymbol{y}_{i} \mid \boldsymbol{\theta}\right)
$$


denota a função de verossimilhança do modelo proposto. Além disto, temos que cada uma das probabilidades $P\left(\boldsymbol{Y}_{i}=\boldsymbol{y}_{i} \mid \boldsymbol{\theta}\right)(i=1, \ldots, n)$ é dada pela seguinte integral múltipla

$$
\int_{A_{i J}} \cdots \int_{A_{i 1}}\left[\int_{0}^{\infty}\left(\int_{\mathbb{R}_{+}^{J}} \phi_{J}\left(\boldsymbol{z} \mid \boldsymbol{X}_{i} \boldsymbol{\beta}+\boldsymbol{D} \boldsymbol{w}, \kappa(\eta) \boldsymbol{\Sigma}\right) \phi_{J}^{+}(\boldsymbol{w} \mid 0, \kappa(\eta) \boldsymbol{I})\right) d H(\eta)\right] d \boldsymbol{z},
$$

em que

$$
B_{i j}= \begin{cases}(0,+\infty) & \text { se } y_{i j}=1 \\ (-\infty, 0] & \text { se } y_{i j}=0\end{cases}
$$

A seguir são apresentadas algumas medidas de comparação de modelos derivadas da função deviance.

\section{Critério de informação de Akaike (AIC) e critério de informação Bayesiano (BIC)}

O critério de informação de Akaike (AIC) e critério de informação Bayesiano (BIC) são medidas de comparação de modelos utilizadas tanto no contexto frequentísta quanto no Bayesiano. Estas medidas são baseadas na função deviance e definidas, respectivamente, por

$$
\mathrm{AIC}=\hat{D}+2 k \text { e } \quad \mathrm{BIC}=\hat{D}+k \log (n)
$$

em que $n$ denota o tamanho da amostra, $k$ denota o número de parâmetros desconhecidos do modelo e $\hat{D}=D(\mathbb{E}[\boldsymbol{\theta} \mid \boldsymbol{y}] ; \boldsymbol{y})$ denota a função deviance avaliada na esperança a posteriori dos parâmetros de interesse. Valores menores de AIC e BIC indicam modelos com melhores ajustes.

\section{Critério de informação da deviance (DIC)}

O critério de informação da deviance (DIC) proposto Spiegelhalter et al. (2002) é o critério mais utilizado de seleção de modelos no contexto Bayesiano. O DIC generaliza os critérios AIC (Akaike, 1973) e BIC (Schwarz, 1978), e é particularmente útil quando as amostras da distribuição a posteriori dos parâmetros de interesse são obtidas através de algoritmos de simulação, por exemplo, o algoritmo de Gibbs. O DIC é definido da seguinte forma

$$
\mathrm{DIC}=2 \hat{D}-\bar{D}
$$

em que $\bar{D}=\mathbb{E}[D(\boldsymbol{\theta} ; \boldsymbol{y}) \mid \boldsymbol{y}]$ denota a esperança a posteriori da função deviance e $\hat{D}$ é mesma quantidade definida em (A.3). Alternativamente, o DIC pode ser reescrito como

$$
\mathrm{DIC}=\hat{D}+2 p_{D}
$$

em que as quantidades $\hat{D}$ e $p_{D}=\bar{D}-\hat{D}$, representam, respectivamente, a qualidade do ajuste do modelo aos dados $(\hat{D})$, e a redução na deviance esperada ao se estimar o vetor de parâmetro de interesse $\boldsymbol{\theta}$. A médida $p_{D}$ também pode ser utilizada como uma estimativa do número efetivo de parâmetros do modelo. Através dos algoritmos MCMC, as quantidades $\bar{D}$ e $\hat{D}$ podem ser estimadas, 
respectivamente, por

$$
\frac{1}{M} \sum_{k=1}^{M} D\left(\boldsymbol{\theta}^{(m)} ; \boldsymbol{y}\right) \text { e } D\left(\frac{1}{M} \sum_{m=1}^{M} \boldsymbol{\theta}^{(m)} ; \boldsymbol{y}\right)
$$

em que $\left\{\boldsymbol{\theta}^{(m)} ; m \leq M\right\}$ representa uma amostra a posteriori do vetor de parâmetros de interesse. O Capítulo 3 apresenta alguns algoritmos MCMC que podem ser utilizados para obtenção desta amostra no modelo proposto.

Valores menores de DIC indicam melhor ajuste. No entanto, Spiegelhalter et al. (2002) argumenta que uma diferença menor que 5 não faz, necessariamente, com que o modelo que apresenta menor DIC deve ser o escolhido. Outros critérios de seleção de modelos devem ser consideradas nesta situação.

O logaritmo da Pseudo-Verossimilhança Marginal (LPML) e o Pseudo-Fator de Bayes (PsBF) são critérios de seleção de modelos computacionalmente atrativos quando a Ordenada Preditiva Condicional (CPO) para cada observação pode ser obtida durante o procedimento de simulação da amostra a posteriori dos parâmetros de interesse via algoritmos de simulação, em particular, os algoritmos MCMC.

\section{Ordenada preditiva condicional (CPO)}

A ordenada preditiva condicional (CPO) (Gelfand e Dey, 1994) é definida como a função de densidade preditiva para a $i$-ésima observação dado o conjunto de dados completo exceto pela pelas variáveis referentes ao $i$-ésimo caso. A estatística CPO para o modelo de regressão multivariado com função de ligação MENA é definido como

$$
P\left(\boldsymbol{Y}_{i}=\boldsymbol{y}_{i} \mid \boldsymbol{y}_{(-i)}\right)=\int P\left(\boldsymbol{Y}_{i}=\boldsymbol{y}_{i} \mid \boldsymbol{\theta}\right) \pi\left(\boldsymbol{\theta} \mid \boldsymbol{y}_{(-i)}\right) d \boldsymbol{\theta}
$$

em que $P\left(\boldsymbol{Y}_{i}=\boldsymbol{y}_{i} \mid \boldsymbol{\theta}\right)$ é definida em (A.2) e $\pi\left(\boldsymbol{\theta} \mid \boldsymbol{y}_{(-i)}\right)$ representa a função de densidade da distribuição a posteriori dos parâmetros de interesse dada todas as observações exceto pelo vetor $\boldsymbol{y}_{i}$. A CPO referente a $i$-ésima observação pode ser escrita como a seguinte esperança harmônica (Chen, Shao e Ibrahim, 2000)

$$
\frac{1}{C P O_{i}}=\int \frac{1}{P\left(\boldsymbol{Y}_{i}=\boldsymbol{y}_{i} \mid \boldsymbol{\theta}\right)} \pi(\boldsymbol{\theta} \mid \boldsymbol{y}) d \boldsymbol{\theta}, i=1, \ldots, n .
$$

Note que a CPO não apresenta uma expressão fácil de ser calculada para o modelo proposto. No entanto, a expressão (A.7) nos permite estimar a CPO através das amostras simuladas

$$
\widehat{C P O}_{i}=\left\{\frac{1}{M} \sum_{m=1}^{M} \frac{1}{P\left(\boldsymbol{Y}_{i}=\boldsymbol{y}_{i} \mid \boldsymbol{\theta}^{(m)}\right)}\right\}^{-1}
$$

em que $\left\{\boldsymbol{\theta}^{(m)} ; m \leq M\right\}$ representada seguinte forma uma amostra a posteriori do vetor de parâmetros de interesse. O Capítulo 3 apresenta alguns algoritmos MCMC que podem ser utilizados para obtenção desta amostra no modelo proposto. 


\section{Logarítmo da pseudo-verossimilhança marginal (LPML)}

Chen, Shao e Ibrahim (2000) sugerem o uso do logaritmo da pseudo-verossimilhança marginal (LPML) como uma medida resumo da CPO sob todas as observações.

$$
L P M L=\sum_{i=1}^{n} \log \left(C P O_{i}\right) .
$$

Neste caso, valores maiores de LPML indicam melhor ajuste.

\section{Pseudo-Fator de Bayes (PsBF)}

O pseudo-Fator de Bayes (PsBF) é uma alternativa ao Fator de Bayes na comparação de modelos quando o cálculo do Fator de Bayes para o modelo proposto é inviável ou quando as CPOs de cada observações já foram calculadas. Para a definição do PsBF, considere $\mathcal{M}_{1}$ e $\mathcal{M}_{2}$ fois modelos concorrentes, o PsBF em favor do modelo $\mathcal{M}_{1}$ em relação ao modelo $\mathcal{M}_{2}$ é definido como

$$
\operatorname{PsBF}\left(\mathcal{M}_{1}, \mathcal{M}_{2}\right)=\frac{\prod_{i=1}^{n} C P O_{i}\left(\mathcal{M}_{1}\right)}{\prod_{i=1}^{n} C P O_{i}\left(\mathcal{M}_{2}\right)}
$$

A interpretação do PsBF como critério de seleção de modelos é a mesma do Fator de Bayes, em que valores de $\operatorname{PsBF}\left(\mathcal{M}_{1}, \mathcal{M}_{2}\right)$ maiores que 1 (um) indicam que o modelo $\mathcal{M}_{1}$ é preferível em relação ao modelo $\mathcal{M}_{2}$.

\section{A.2 Análise de observações influentes}

A análise de diagnóstico é uma etapa muito importante no processo de modelagem. Nesta etapa verifica-se a coerência das suposições consideradas para o modelo proposto e avalia-se a qualidade do ajuste. O diagnóstico de influência é uma das ferramentas mais utilizadas para a verificação da qualidade do ajuste do modelo proposto aos dados. Estes métodos visam a identificação de observações ou conjuntos de observações que apresentam influência desproporcional nos resultados inferenciais, e por isto, merecem maior atenção e consideração. A forma mais simples de detectar tais observações é o de exclusão de casos (Cook, 1977). Este método, chamado de influência global, consiste em excluir um subconjunto de observações, reajustar o modelo e verificar as variações nas estimativas do parâmetros. O afastamento da verossimilhança (LD) é a medida de influência mais conhecida para avaliar o impacto de observações influentes em um modelo estatístico. O LD também pode ser utilizado para verificar o impacto de outras perturbações além da exclusão de casos (Cook, 1986). No entanto, nós apresentamos a seguir o afastamento da verossimilhança (LD) definido apenas para caso em que a $i$-ésima observação é excluída,

$$
L D\left(\boldsymbol{y}_{i}\right)=2\left\{\log [L(\boldsymbol{\theta} ; \boldsymbol{y})]-\log \left[L\left(\hat{\boldsymbol{\theta}}_{(-i)} ; \boldsymbol{y}_{(-i)}\right)\right]\right\}
$$

em que $\log [L(\boldsymbol{\theta} ; \boldsymbol{y})]$ denota o logaritmo da verossimilhança do modelo proposto avaliada no estimador de máxima verossimilhança $\hat{\boldsymbol{\theta}}$ e $\log \left[L\left(\hat{\boldsymbol{\theta}}_{(-i)} ; \boldsymbol{y}_{(-i)}\right)\right]$ denota o o logaritmo da verossimilhança 
avaliada sob o modelo em que $\boldsymbol{\theta}_{(-i)}$ é o estimador de máxima verossimilhança de $\boldsymbol{\theta}$ com a exclusão do $i$-ésimo caso.

\section{O inverso da CPO}

No contexto Bayesiano, o inverso da estatística $\mathrm{CPO}_{i}$ apresentada na expressão (A.7) pode ser utilizada para verificar se a $i$-ésima observação de um particular conjunto de dados pode ser considerada uma observação atípica no modelo proposto. Por construção, temos que valores altos para a $\mathrm{CPO}_{i}$, e consequentemente valores baixos para o $\mathrm{CPO}_{i}^{-1}$, implica maior concordância entre a $i$-ésima observação e o resto dos dados, e portanto um melhor ajuste. $\mathrm{O}^{\mathrm{CPO}_{i}^{-1}}$ pode ser estimado da seguinte forma.

$$
\widehat{C P O}_{i}^{-1}=\frac{1}{M} \sum_{m=1}^{M} \frac{1}{P\left(\boldsymbol{Y}_{i}=\boldsymbol{y}_{i} \mid \boldsymbol{\theta}^{(m)}\right)},
$$

em que $\left\{\boldsymbol{\theta}^{(m)} ; m \leq M\right\}$ representa uma amostra a posteriori do vetor de parâmetros de interesse. O Capítulo 3 apresenta alguns algoritmos MCMC que podem ser utilizados para obtenção desta amostra no modelo proposto.

\section{A divergência de Kullback-Leibler (KL)}

Cho et al. (2009) cita diversos autores que utilizaram a informação (divergência) de KullbackLeibler (divergência K-L) como uma medida de discrepância entre distribuições a posteriori para realização de diagnóstico de influência na situação de deleção de casos (observações). A seguinte divergência K-L quantifica a discrepância entre as distribuições a posteriori $\pi(\theta \mid \boldsymbol{Y})$ e $\pi\left(\theta \mid \boldsymbol{Y}_{i}\right)$

$$
K\left[\pi, \pi_{(-i)}\right]=\int \log \left\{\frac{\pi(\boldsymbol{\theta} \mid \boldsymbol{y})}{\pi\left(\boldsymbol{\theta} \mid \boldsymbol{y}_{(-i)}\right)}\right\} \pi(\boldsymbol{\theta} \mid \boldsymbol{y}) d \boldsymbol{\theta},
$$

em que $\pi=\pi(\theta \mid \boldsymbol{y})$ denota a distribuição a posteriori do vetor de parâmetros $\boldsymbol{\theta}$ para todos os dados e $\pi_{(-i)}=\pi\left(\theta \mid \boldsymbol{y}_{(-i)}\right)$ denota a distribuição a posteriori do vetor de parâmetros $\boldsymbol{\theta}$ para todos os dados exceto pela $i$-ésima observação. A divergência $K\left[\pi, \pi_{(-i)}\right]$ representa o impacto que ocorre ao deletar a $i$-ésima observação do conjunto de dados na distribuição a posteriori do vetor de parâmetros $\boldsymbol{\theta}$. A divergência $K\left[\pi, \pi_{(-i)}\right]$ pode ser vista como uma analogia bayesiana do afastamento da verossimilhança apresentado na expressão (A.11). Ao longo do texto os termos divergência e discrepância são utilizados para definir a medida $K\left[\pi, \pi_{(-i)}\right]$. Isto ocorre porque a medida de $\mathrm{K}-\mathrm{L}$ não é uma medida de distância, pois $K\left[\pi, \pi_{(-i)}\right]$ pode ser diferente de $K\left[\pi_{(-i)}, \pi\right]$.

Cho et al. (2009) apresentam uma expressão simplificada para calcular a divergência K-L em função da estatística CPO,

$$
K\left[\pi, \pi_{(-i)}\right]=\mathbb{E}_{\boldsymbol{\theta}}\left\{\log \left[P\left(\boldsymbol{Y}_{i}=\boldsymbol{y}_{i} \mid \boldsymbol{\theta}\right)\right]\right\}-\log \left(C P O_{i}\right),
$$

em que $\mathbb{E}_{\boldsymbol{\theta}}\left\{\log \left[P\left(\boldsymbol{Y}_{i}=\boldsymbol{y}_{i} \mid \boldsymbol{\theta}\right)\right]\right\}$ representa a esperança a posteriori da função $\log \left[P\left(\boldsymbol{Y}_{i}=\boldsymbol{y}_{i} \mid \boldsymbol{\theta}\right)\right]$. A expressão (A.13) nos permite estimar a divergência $K\left[P, P_{(-i)}\right]$ através das amostras dos parâmetros 
de interesse obtidas no processo de simulação das amostras a posteriori da seguinte forma.

$$
\widehat{K}\left[\pi, \pi_{(-i)}\right]=\frac{1}{M} \sum_{m=1}^{n} \log \left[P\left(\boldsymbol{Y}_{i}=\boldsymbol{y}_{i} \mid \boldsymbol{\theta}^{(m)}\right)\right]-\log \left(\widehat{C P O}_{i}\right), i=1, \ldots, n,
$$

em que $\left\{\boldsymbol{\theta}^{(m)} ; m \leq M\right\}$ representa uma amostra a posteriori do vetor de parâmetros de interesse. O Capítulo 3 apresenta alguns algoritmos MCMC que podem ser utilizados para obtenção desta amostra no modelo proposto.

\section{Calibração da divergência de Kullback-Leibler}

Cho et al. (2009) também apresentam uma forma de calibrar a divergência $K\left(\pi, \pi_{(-i)}\right)$. Esta calibração é obtida resolvendo para $p_{i}$ a seguinte expressão

$$
K\left[\pi, \pi_{(-i)}\right]=K\left[\operatorname{Ber}(1 / 2), \operatorname{Ber}\left(p_{i}\right)\right]=\frac{1}{2} \log \left[4 p_{i}\left(1-p_{i}\right)\right],
$$

em que $\operatorname{Ber}(p)$ denota a distribuição de Bernoulli com probabilidade de sucesso $p$.

Note que considerar a expressão A.15 verdadeira implica assumir que as distribuições a posteriori $\pi(\boldsymbol{\theta} \mid \boldsymbol{y})$ e $\pi\left(\boldsymbol{\theta} \mid \boldsymbol{y}_{(-i)}\right)$ estão tão próximas quanto as distribuições $\operatorname{Ber}(1 / 2)$ e $\operatorname{Ber}(p)$. Portanto, uma observação que faz a diferença entre as distribuições a posteriori $\pi\left(\boldsymbol{\theta} \mid \boldsymbol{y}_{(-i)}\right)$ e $\pi(\boldsymbol{\theta} \mid \boldsymbol{y})$ ser grande vai ser refletida na probabilidade de sucesso $p_{i}$, fazendo com que $p_{i}$ seja igual a 1 (um) quando a diferença entre as distribuições a posteriori for máxima. A calibração é dada por

$$
p_{i}=\frac{1}{2}\left[1+\sqrt{1-\exp \left\{-2 K\left[\pi, \pi_{(-i)}\right]\right\}}\right] .
$$

Esta expressão implica que $0,5 \leq p_{i} \leq 1$. Os autores sugerem considerar a $i$-ésima observação influente quando $p_{i}$ for muito maior que $0,5, i=1, \ldots, n$, pois deletar a $i$-ésima observação provoca uma mudança na distribuição a posteriori tão grande quanto considerar um evento tem probabilidade $p_{i}$ quando a probabilidade correta é 0,5 . 


\section{Referências Bibliográficas}

Akaike, H. (1973). Information theory and an extension of the maximum likelihood principle. In: Petrov, B; Csaki, F. (Org.). International Symposium on Information Theory. Budapest Kiado, p.267-281. 68

Albert, J. H. e Chib, S. (1993). Bayesian analysis of binary and polychotomous response data. Journal of the American Statistical Association, 88, 669-979. 2, 8, 12, 17, 27

Andrews, D. F. e Mallows, C. L. (1974). Scale mixtures of normal distributions. J. Roy. Statist. Soc. Ser. B, $\mathbf{3 6}, 99-102.2,13,20$

Arellano-Valle, R. (1994). Distribuções elípticas: propriedades, inferência e aplicações a modelos de regressão, PhD Thesis, IME-USP, São Paulo. 10

Arslan, O. (2008). An alternative multivariate skew-slash distribution. Statistics and Probability Letters, 78, 27f6-2761. 19

Azzalini, A. (1985). A class of distributions which includes the normal ones. Scand .J.Statist. 12, $171-17814$

Azzalini, A. e Dalla-Valle, A. (1996). The multivariate skew-normal distribution. Biometrika, 83, $715-72614$

Barnard, J., McCulloch, R. e X. Meng, X. 2000. Modeling covariance matrices in terms of standard deviations and correlations, with application to shrinkage. Statistica Sinica, 10, 1281-1311. 30, 31,32

Bazán, J. L., Branco, M. D. e Bolfarine, H. (2005). A skew item response model. Bayesian Analysis, 1, 861-892. 3

Bazán, J. L., Bolfarine, H. e Branco, M. D. (2010), A framework for skew-probit links in binary regression, Communication in Statistics - Theory and Methods. 39, 678-697 . 3

Branco, M. D. e Dey, D. K.(2001). A class of multivariate skew-elliptical distributions, Journal of Multivariate Analysis, 79, 99-113. 14, 15

Bliss, C. I. (1935). The calculation of the dose-mortality curve, Annals Applied Biology. 22, 134-167. 3

Carlin, B. P, e Louis, T. A. (1996). Bayes and Empirical Bayes Methods for Data Analysis. London: Chapman \& Hall. 26 
Cancho, V. G., Ortega, E. M. M. e Paula, G. A. (2010). On estimation and influence diagnostics for log-Birnbaum-Saunders regression models. Journal of Statistical Planning and Inference 140, 2486-2496 56

Carey, V., Zeger, S. L. e Diggle, P. (1993). Modeling multivariate binary data with alternating logistic regressions.Biometrika. 80, 517-26. 2

le Cessie, S. e van Houwelingen, J.C. (1994). Logistic Regression for Correlated Binary Data. Applied Statistics 43, 95-108. 2

Chen, M-H. (2004). Skewed link models for categorical response data. In Skew-Elliptical Distributions and Their Applications: A Journey Beyond Normality. Genton, M. G., Ed., Chapman and Hall / CRC, Boca Raton, FL, pp. 131-151. 3

Chen, M-H. e Dey, D. K. (1998). Bayesian modeling of correlated binary response via scale mixture of multivariate normal link functions. Sankhyã 60, 322-343. 2, 13, 22, 25, 29, 34, 35, 36, 65

Chen, M-H. e Dey, D. K. (2000). Bayesian Analysis for correlated ordinal data model. In Generalized Linear Models: A Bayesian Perspective. Dey, D.K., Gosh, S.K. e Malick, B. K. (eds). New York: Marcel Dekker. 13, 65

Chen, M. H., Dey, D. K., e Shao, Q-M. (1999). A new skewed link model for dichotomous quantal response data. Journal of the American Statistical Association, 94, 448, 1172-1186. 2, 3

Chen, M-H. e Dey, D. K. (2003). Variable selection for multivariate logistic regression models. Journal of Statistical Planning and Inference, 111, 37-55. 2, 4, 8, 27, 29

Chen, M-H., Huang, L., Ibrahim, J. G.e Kim, S (2008). Bayesian Variable Selection and Computation for Generalized Linear Models with Conjugate Priors. Bayesian Analysis, 3, 585-614.

Chen, M., Shao, Q. e Ibrahim, J.(2000). Monte Carlo Methods in Bayesian Computation. SpringerVerlag. NY 56

Chib, S. (1999). Bayesian Methods for Correlated Binary Data. In Generalized Linear Models: A Bayesian Perspective. Dey, D.K., Gosh, S.K. e Malick, B. K. (eds). New York: Marcel Dekker, pp. 113-131. 69, 70

Chib, S. e Carlin, B. P. (1999). On MCMC sampling in hierarchical longitudinal models. Statistics and Computing, 9, 17-26. 10

Chib, S. e Greenberg, E. (1998). Analysis of multivariate probit models. Biometrika, 85, 347-361.

Cho, H., Ibrahim, J. G., Sinha, D.e Zhu, H. (2009). Bayesian Case Influence Diagnostics for Survival Models, Biometrics, 65, 116-124. 2, 4, 8, 10, 22, 25, 27, 29, 49, 50, 52, 65

Choy, S. T. B. (1995). Robust Bayesian analysis using scale mixture of normals distributions. Ph.D. Dissertation,. Department of Mathematics. Imperial College 71, 72

Cook, R. D. (1977). Detection of influential observations in linear regression. Technometrics, 19, 15-18. 19, 20 
Cook, R. D. (1986). Assessment of local influence. Journal of the Royal Statistical Society, Series $B, 48,133-169.70$

Cowles, M. K. (1996). Accelerating Monte Carlo Markov Chain convergence for cumulative-link generalized linear models. Statistics and Computing, 6, 101-111. 70

2

Czado, C. e Santner, T. J. (1992). The effect of link misspecification on binary regression inference, Journal of Statistical Planning and Inference, 33, 213-231. 3

Dempster, A. P., Laird, N. M. e Rubin, D. B. (1977). Maximum likelihood from incomplete data via the EM algorithm. Journal of Royal Statistical Society. Series B 39, 1-38 8

Doornik, J. A. (2006). Ox: An Object-Oriented Matrix Language. Timberlake Consultants Press, London. 5 th ed. $39,40,50,54,66$

Fang, K. e Anderson, T. (1990). Statistical Inference in Elliptically Contoured and Related Distributions, Allerton Press, New York. 10

Fang, K. T., Kotz, S. e Ng, K. W. (1990). Symmetric Multivariate and Related Distributions, Chapman and Hall. 10

Farias, R. B. A. Branco, M. D. (2011). Efficient Algorithms for Bayesian Binary Regression Model with Skew-Probit Link. In: Manish Bhattacharjee; Sunil K Dhar; Sundarraman Subramanian. (Org.). Recent Advances in Biostatistics. : World Scientific Publishing, v.4, p.143-168. 2

Farias, R. B. A. Branco, M. D. (2012). Latent residual analysis in binary regression with skewed link. Revista Brasileira de Probabilidade e Estatistica. A aparecer. 2

Farias, R. B. A. e Lemonte, A. J. (2011). Bayesian inference for the Birnbaum Saunders nonlinear regression model. Statistical Methods and Applications, 20, p. 423-438. 56

Gamerman, D. e Lopes, H. (2006). Monte Carlo Markov Chain: Stochastic Simulation for Bayesian Inference. Chapman and Hall.

Gelfand, A. E. e Dey, D. K. (1994). Bayesian Model Choice: Asymptotics and Exact Calculations.Journal of the Royal Statistical Society, Series B, 56. 501-514. 69

Gelfand, A. E. e Smith, A. F. M. (1990). Sampling-based approaches to calculating marginal densities. Journal of the American Statistical Association, 85, 398-409. 2

Gelman, A., Carlin, J. B., Stern, H. S. e Rubin, D. B. (2003). Bayesian Data Analysis, 2th ed. Chapman \& Hall/CRC, Boca Raton, FL. 67

Genton, M. G. (2004),Skew-Elliptical Distributions and Their Applications: A Journey Beyond Normality Edited Volume, Chapman \& Hall/CRC, Boca Raton, FL. 14

Genz, A e Azzalini, A. (2011). mnormt: The multivariate normal and t distributions. R package version 1.4-0. 66 
Glonek, G. F. V. e McCullagh, P. (1995). Multivariate Logistic Models. J. Roy. Statist. Soc. Ser. $B, 57,533-546.2$

Greene, W. H. (1997). Econometric Analysis, 3th ed., New Jersey: Prentice Hall. 1, 49, 50, 52

Holmes, C. C., e Held, L. (2006). Bayesian auxiliary variable models for binary and multinomial regression, Bayesian Analysis, 1, 145-168. 2, 35

Johnson, W. O., Gastwirth, J. L. and Pearson, L. M. (2001). Screening without a "gold-standard": The Hui-Walter paradigm revisited. American J. Epidemiology, 153, 921-924. 26

Kim, H-M (2008). On note on scale mixtures of skew normal distribution, Statistics and Probability Letters, 78, 1694-1701. 15

Liang, K. Y. e Zeger, S. L. (1986). Longitudinal data analysis using generalized linear models, Biometrika, 73(1), 13-22. 2

Liu, J. The collapsed Gibbs sampler in Bayesian computations with applications to a gene regulation problem, Journal of the American Statistical Association, 89, 978-966. 2

Liu, J. e Wu, Y. (1999). Parameter expansion for data augmentation, Journal of the American Statistical Association, 94, 1264-1274. 30, 31

McCulloch, R e Rossi, P. (1994). An exact likelihood analysis of the multinomial probit model, Journal of Econometrics, 64(1-2), 207-240. 25

McCulloch, R., Polson, N. e Rossi, P. (2000). A Bayesian analysis of the multinomial probit model with fully identified parameters, Journal of Econometrics, 99(1), 173-193. 25

Tierney, L. (1994) Markov chains for exploring posterior distributions (with discussion). Annals of Statistics, 22, 1701-1762

Nandram, B. e Chen M. H.(1996). Reparameterizing the generalized linear model to accelerate Gibbs sampler convergence. Journal of Statistical Computation and Simulation. 54, 129-144. 2

Nobile, A. (2000). Comment: Bayesian multinomial probit models with a normalization constraint, Journal of Econometrics, 99(2), 335-345. 25

Paulino, C. D. Turkman, M. A. e Murteira, B. (2003). Estatística Bayesiana. Fundação Calouste Gulbenkian, Lisboa. 39

Plummer, M., Best, N., Cowles, K., Vines, K. (2006). CODA: Convergence diagnosis and output analysis for MCMC. $R$ News 6, 7-11. 50, 54, 66

Prentice, R. L.(1976). A generalization of the probit and logit methods for dose-response curves, Biometrics, 32, 761-768. 3

Prentice, R. L.(1988). Correlated binary regression with covariates specific to each binary observation, Biometrics, 44, 1033-48 2

R Development Core Team (2009). R: A Language and Environment for Statistical Computing. Vienna, Austria. 66 
Rannala, B. (2002). Identifiability of parameters in MCMC Bayesian inference of phylogeny. Systematic Biology, 51, 754-760 26

Sahu, S. K., Dey, D. K. e Branco, M. D.(2003). A new class of multivariate skew distributions with applications to Bayesian regression Models, The Canadian Journal of Statistics, 31, 129-150. 14, $15,16,18,27,28,53,65$

Schwarz, G. (1978). Estimating the Dimension of a Model. The Annuals of Statistical, 6, 461-464. 68

Shariati, M. M., Korsgaard, I. R, e Sorensen, D. (2009) Identifiability of parameters and behaviour of MCMC chains: a case study using the reaction norm model. Journal of animal breeding and genetics Zeitschrift fur Tierzuchtung und Zuchtungsbiologie. 126, 92-102. 26

Spiegelhalter, D. J., Best, N. G., Carlin, B. P. e van der Linde, A. (2002). Bayesian measures of model complexity and fit. Journal of the Royal Statistical Society, Series B, 64, 583`640. 54, 68, 69

Stukel, T.(1988). Generalized logistic models, Journal of the American Statistical Association, 83, 426-431. 3

Swartz, T. B., Haitovsky, Y., Vexler, A. e Yang, T. Y. (2004). Bayesian identifiability and misclassification in multinomial data. Canadian Journal of Statistics. 32, 285-302. 26

Tabet, A. (2007). Bayesian inference in the multivariate probit model. Master Thesis, The University Of British Columbia, Columbia. 30, 31, 32

Vernic, R (2005). On the multivariate Skew-Normal distribution and its scale mixtures. An. Stiint. Univ. "Ovidius"Constanta, Ser. Mat. 13, 2, 83-96. 15

Wang, J e Genton, M. G. (2006). The Multivariate skew-slash distribution. Journal of Statistical Planning and Inference, 136, 209-220. 19

Wang, X e Dey, D. K. (2010). Generalized extreme value regression for binary response data: An application to B2B electronic payments system adoption. The Annals of Applied Statistics, 4, 2000-2023. 3

Webb, E. L. e Forster, J. J. (2008). Bayesian model determination for multivariate ordinal and binary data, Comput. Stat. Data Anal., 52, 2632-2649.

West, M. (1987). On scale mixtures of normal distributions, Biometrika, 74, 646-648. 13, 20

Zeger, S. L. e Liang, K. Y. (1986). Longitudinal data analysis for discrete and continuous outcomes. Biometrics, 42, 121-130. 2

Zellner, A. e Rossi, P. E.(1984). Bayesian analysis of dichotomous quantal response models, Journal of Econometrics, 25, 365-393. 2 
